\title{
Kinser inequalities and related matroids
}

\author{
Amanda Cameron
}

\section{VICTORIA UNIVERSITY OF WELLINGTON \\ Te Whare Wananga o te Upoko o te Ika a Maui}

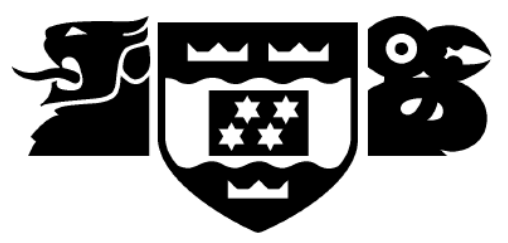

School of Mathematics, Statistics and Operations Research

Te Kura Mātai Tatauranga, Rangahau Pūnaha

\author{
A thesis \\ submitted to the Victoria University of Wellington \\ in fulfilment of the requirements for the degree of \\ Master of Science \\ in Mathematics.
}

Victoria University of Wellington

2013 


\begin{abstract}
Kinser developed a hierarchy of inequalities dealing with the dimensions of certain spaces constructed from a given quantity of subspaces. These inequalities can be applied to the rank function of a matroid, a geometric object concerned with dependencies of subsets of a ground set. A matroid which is representable by a matrix with entries from some finite field must satisfy each of the Kinser inequalities. We provide results on the matroids which satisfy each inequality and the structure of the hierarchy of such matroids.
\end{abstract}




\section{Acknowledgements}

I would like to thank Dillon Mayhew for his advice, encouragement and supervision.

Thanks also go to Michael Welsh for technical support and for proof-reading this thesis. 


\section{Contents}

1 Introduction 1

\begin{tabular}{|lll}
2 & Fundamentals & 4
\end{tabular}

$2.1 \quad$ Dependencies . . . . . . . . . . . . . . . . 5

2.2 Representability . . . . . . . . . . . . . . . 6

2.3 Minors . . . . . . . . . . . . . . . . 6

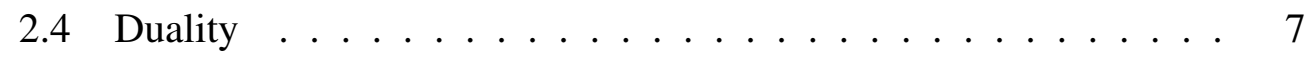

2.5 Transversals . . . . . . . . . . . . . . . . . 7

2.6 Free extensions . . . . . . . . . . . . . . . . . 8

3 Kinser Inequalities 9

$3.1 \quad$ Inequalities $\ldots \ldots \ldots \ldots \ldots$

3.2 Kinser matroids . . . . . . . . . . . . . . . . . . 13

3.3 Kinser classes $\ldots \ldots \ldots \ldots \ldots$

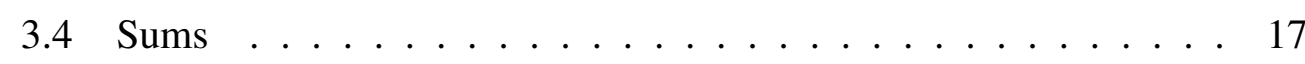

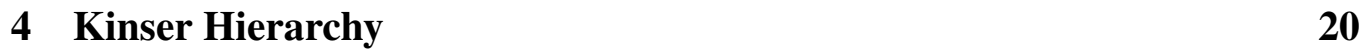

\begin{tabular}{lll}
\hline 5 & $\mathcal{K}_{5} \neq \mathcal{K}_{5}^{*}$ & 30
\end{tabular}

6 A Complexity Theorem $\quad 60$

\begin{tabular}{lll}
\hline 7 & Excluded minors & 63
\end{tabular} 
\begin{tabular}{|ll|}
\hline Conjectures & 71
\end{tabular}

\begin{tabular}{ll}
\hline Bibliography & 75
\end{tabular} 


\section{List of Figures}

$3.1 \quad \operatorname{Kin}(4) \ldots \ldots \ldots \ldots \ldots$

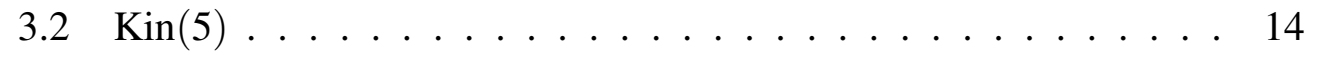

$4.1 \quad$ Kinser classes $\ldots \ldots \ldots . \ldots 22$

4.2 Kinser classes $(2) \ldots \ldots \ldots \ldots$

$4.3 \quad$ Kinser classes (3a) $\ldots \ldots \ldots$

$4.4 \quad$ Kinser classes $(3 \mathrm{~b})$

$8.1 \quad$ Kinser classes $(4)$. . . . . . . . . . . . . . . . . 72 


\section{Chapter 1}

\section{Introduction}

A fundamental question in matroid theory is whether it is possible to find a characterisation of the class of representable matroids. In particular, we wish to know whether this can be achieved with a finite number of axioms, by adding additional rank axioms to the existing three. This was first alluded to by Whitney [10], in the paper which initiated the area of matroid theory, and the problem remains open today. Ingleton [3] introduced one new axiom which a matroid must satisfy in order to be representable.

Definition 1.1. Let $M=(E, r)$ be a matroid. For subsets $X_{1}, \ldots, X_{4}$ of $E$, the Ingleton inequality is:

$$
\begin{aligned}
& r\left(X_{3}\right)+r\left(X_{4}\right)+r\left(X_{1} \cup X_{2}\right)+r\left(X_{1} \cup X_{3} \cup X_{4}\right)+r\left(X_{2} \cup X_{3} \cup X_{4}\right) \\
\leq & r\left(X_{1} \cup X_{3}\right)+r\left(X_{1} \cup X_{4}\right)+r\left(X_{2} \cup X_{3}\right)+r\left(X_{2} \cup X_{4}\right)+r\left(X_{3} \cup X_{4}\right)
\end{aligned}
$$

This new condition, while necessary, is not sufficient to characterise representability. For instance, the direct sum of the Fano and non-Fano matroids satisfies the Ingleton condition but is not representable, as later proved in Lemma 4.1. 


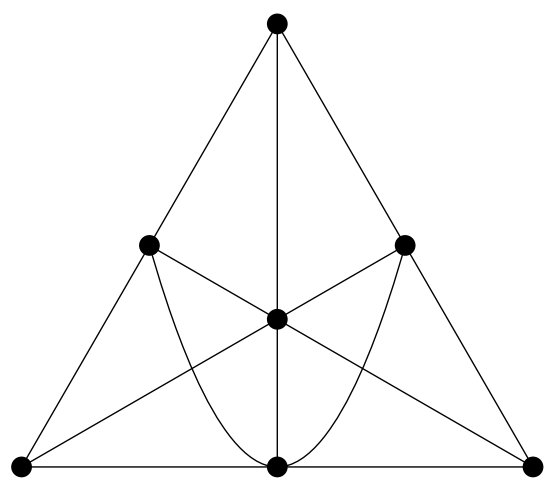

Fano matroid, $F_{7}$

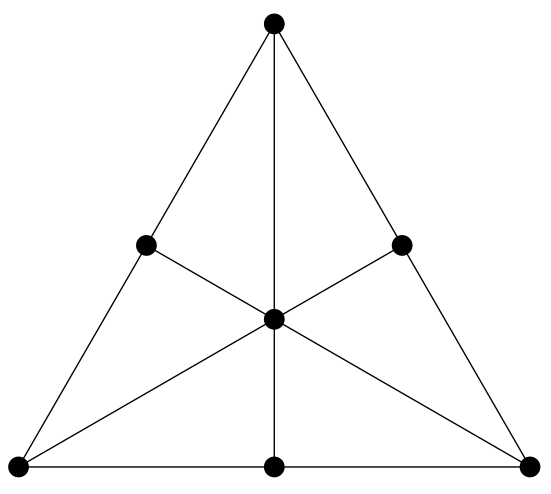

Non-Fano matroid, $F_{7}^{-}$

More recently, Kinser [4] introduced an infinite family of new representability conditions, the first of which is equivalent to the Ingleton condition.

Definition 1.2. Let $M$ be a matroid, and let $X_{1}, \ldots, X_{n}$ be any collection of subsets of $E(M)$. The $n$-th Kinser inequality, where $n \geq 4$, is

$$
\begin{aligned}
& \sum_{i=3}^{n} r\left(X_{i}\right)+r\left(X_{1} \cup X_{2}\right)+r\left(X_{1} \cup X_{3} \cup X_{n}\right)+\sum_{i=4}^{n} r\left(X_{2} \cup X_{i-1} \cup X_{i}\right) \\
& \quad \leq r\left(X_{1} \cup X_{3}\right)+r\left(X_{1} \cup X_{n}\right)+\sum_{i=3}^{n} r\left(X_{2} \cup X_{i}\right)+\sum_{i=4}^{n} r\left(X_{i-1} \cup X_{i}\right)
\end{aligned}
$$

This hierarchy of inequalities is also not sufficient to guarantee representability of a matroid - the direct sum of the Fano and the non-Fano is again a counterexample to this. Briefly putting aside the use of an infinite list of axioms, we have the following conjecture, which is due to Mayhew, Newman, and Whittle [5].

Conjecture 1.3. It is impossible to characterise the class of representable matroids with a finite number of rank axioms.

Note that [5] is a response to an paper of Vámos' [9] dealing with the same question. In this paper, Vámos introduced the following geometric construction, which we call a $V$-matroid:

Definition 1.4. A $V$-matroid consists of a (possibly infinite) set $E$ and a collection of finite subsets $\mathcal{I} \subseteq E$ such that:

I1. $\varnothing \in \mathcal{I}$

I2. If $I \in \mathcal{I}$ and $J \subseteq I$, then $J \in \mathcal{I}$ 
I3. If $I, J \in \mathcal{I}$ and $|I|=|J|+1$, there exists $x \in I-J$ such that $J \cup x \in \mathcal{I}$

Instead of using rank axioms, Vámos describes $V$-matroids with an infinite list of first-order axioms. In [9], Vámos proved that it is not possible to characterise representable $V$-matroids by adding a further first-order axiom to this infinite list. Note that a first-order axiom is not equivalent to a rank axiom. However, as every finite $V$-matroid is a matroid, Conjecture 1.3 could be regarded as having been inspired by this result.

This thesis is dedicated to investigating the classes of matroids which satisfy each of the Kinser inequalities. We will cover invariant properties of the classes, such as being minor-closed and direct sum closed, and, more importantly, we will provide results on how the classes interact with each other to form an infinite hierarchy. We will touch on the complexity of verifying a matroid satisfies a given Kinser inequality, which will show that gaining certain information on the Kinser classes, such as which classes are closed under duality, could involve a great amount of computational work.

This thesis will conclude by considering a question which arises naturally in conjunction with representability, that of excluded minors. The following theorem was proved by Mayhew, Newman, and Whittle in 2008 [6], settling a conjecture by Geelen [2].

Theorem 1.5. For any infinite field $\mathbb{K}$ and any matroid $N$ representable over $\mathbb{K}$, there is an excluded minor for $\mathbb{K}$-representability that has $N$ as a minor.

We will provide a strengthening of this result, showing that there is in fact an infinite number of such excluded minors. Specifically, we will show that for each layer of the Kinser class hierarchy, we can find an excluded minor which is contained inside that layer. 


\section{Chapter 2}

\section{Fundamentals}

To begin with, we will cover the basic concepts in matroid theory which will be used throughout this thesis. All of the following concepts and results can be found in [7].

Definition 2.1. A matroid $M=(E, \mathcal{I})$ consists of a finite ground set $E$ and a collection of subsets $\mathcal{I} \subseteq E$ such that:

I1. $\varnothing \in \mathcal{I}$

I2. If $I \in \mathcal{I}$ and $J \subseteq I$, then $J \in \mathcal{I}$

I3. If $I, J \in \mathcal{I}$ and $|I|<|J|$, there exists $x \in J-I$ such that $I \cup\{x\} \in \mathcal{I}$

Any subset of $E$ contained in $\mathcal{I}$ is referred to as an independent set, while any subset of $E$ which is not contained in $\mathcal{I}$ is called dependent. A dependent set of cardinality one is called a loop. We may use $E(M)$ in the place of $E$ at times, in order to make it clear which matroid is being referred to.

Definition 2.2. Take a matroid $M$ with ground set $E$. The rank of a subset $X$ of $E$, denoted by $r(X)$, is the cardinality of the largest independent subset of $X$.

Lemma 2.3. A matroid $M$ can be described by the ground set $E$ and $a$ rank function $r: \mathcal{P}(E) \rightarrow \mathbb{Z}^{+} \cup\{0\}$ such that, for $X, Y \in \mathcal{P}(E)$, the following conditions hold:

R1. $r(X) \leq|X|$ 
R2. If $Y \subseteq X, r(Y) \leq r(X)$

R3. $r(X \cup Y)+r(X \cap Y) \leq r(X)+r(Y)$

A set $X$ is independent if and only if $r(X)=|X|$. If $r(X)=r(M)$ we call $X$ a basis of $M$. If a set contains a basis, it is called spanning.

\subsection{Dependencies}

Definition 2.4. The closure of a set $X$ is denoted by $\operatorname{cl}(X)$, where

$$
\operatorname{cl}(X)=X \cup\{e \in E-X \mid r(X \cup e)=r(X)\}
$$

Lemma 2.5. The closure function of a matroid satisfies the following conditions:

CL1. If $X \subseteq E$, then $X \subseteq \operatorname{cl}(X)$.

CL2. If $X \subseteq Y$, then $\operatorname{cl}(X) \subseteq \operatorname{cl}(Y)$.

CL3. If $X \subseteq E$, then $\operatorname{cl}(\operatorname{cl}(X))=\operatorname{cl}(X)$.

CL4. If $X \subseteq E$ and $x \in E$, and $y \in \operatorname{cl}(X \cup x)-\operatorname{cl}(X)$, then $x \in \operatorname{cl}(X \cup y)$.

The closure function corresponds to the notion of span of a vector space, and is sometimes referred to as such. A flat is a set whose closure is equal to the set itself, i.e. $\operatorname{cl}(X)=X$. If a flat has rank $r(M)-1$, it is called a hyperplane.

A minimally dependent set - that is, a dependent set where every proper subset of that set is independent - is called a circuit. A matroid can be described entirely by its set of circuits $\mathcal{C}$.

Lemma 2.6. $(E, \mathcal{C})$ describes a matroid when the following conditions hold.

C1. $\varnothing \notin \mathcal{C}$

C2. If $C, D \in \mathcal{C}$ and $C \subseteq D$, then $C=D$

C3. If $C, D$ are distinct elements of $\mathcal{C}$ amd $e \in C \cup D$, then $(C \cup D)-e$ contains a circuit 
A circuit-hyperplane is a set which is both a circuit and a hyperplane.

Definition 2.7. Let $M$ be a matroid and let $H$ be a circuit-hyperplane of $M$. $H$ has rank equal to $r(M)-1$. We say that we relax $H$ when we make it independent, i.e. $r(H)=r(M)$. When we reverse this operation, we say that we tighten $H$.

\subsection{Representability}

Definition 2.8. If $V$ is a set of vectors in a vector space, and for every subset $X$ of $V$, we define $r(X)$ to be the linear rank of $X$, then $(V, r)$ is a matroid, which we say is representable.

If these vectors come from a finite field $\mathbb{K}$, we say that $M$ is $\mathbb{K}$-representable.

\subsection{Minors}

Definition 2.9. We can remove an element $e$ of a matroid $M=(E, r)$ by deleting it. This yields a matroid $M \backslash e=\left(E-\{e\}, r_{M \backslash e}\right)$, where $r_{M \backslash e}(X)=r_{M}(X)$ for all $X \subseteq E-\{e\}$.

Definition 2.10. We can also remove an element $e$ of a matroid $M=(E, r)$ by contracting it. This gives a matroid $M / e=\left(E-\{e\}, r_{M / e}\right)$ where $r_{M / e}(X)=$ $r_{M}(X \cup\{e\})-r(\{e\})$ for all $X \subseteq E-\{e\}$.

Any matroid produced by a sequence of deletions and contractions is called a minor of $M$.

We say that a class of matroids $\mathcal{M}$ is minor-closed if, for every matroid $M$ in $\mathcal{M}$, each of its minors is also in $\mathcal{M}$.

A matroid $M$ is an excluded minor for a minor-closed class of matroids $\mathcal{M}$ if $M \notin \mathcal{M}$ but deleting or contracting any element from $M$ produces a matroid in $\mathcal{M}$. A matroid $M$ is contained in $\mathcal{M}$ if and only if $M$ does not contain an excluded minor for $\mathcal{M}$. 


\subsection{Duality}

Definition 2.11. From $M$ we can construct the dual matroid $M^{*}$. This has ground set equal to the ground set $E$ of $M$, and the rank of any subset $X$ is found using the function $r^{*}(X)=|X|+r\left(E^{*}-X\right)-r(M)$.

A basis of $M^{*}$ is is called a cobasis of $M$. Note that if $B$ is a basis of $M$, then $E-B$ is a cobasis of $M$. Similarly, the rank function, circuits, loops and independent sets of $M^{*}$ are called the corank function, cocircuits, coloops and coindependent sets of $M$.

Lemma 2.12 ([7, Proposition 2.1.7]). Let $M$ be a matroid. Relax a circuithyperplane $H$ of $M$ to yield the matroid $M^{\prime}$. Then $\left(M^{\prime}\right)^{*}$ is identical to the matroid yielded from $M^{*}$ by relaxing the circuit-hyperplane $E-H$ of $M^{*}$.

Lemma 2.13 ([7, Proposition 3.3.5]). Let $H$ be a circuit-hyperplane of a matroid $M$, and let $M^{\prime}$ be the matroid obtained from $M$ by relaxing $H$.

i. When $e \in E(M)-H, M / e=M^{\prime} / e$, and, unless $e$ is a coloop of $M, M^{\prime} \backslash e$ is obtained from $M \backslash$ e by relaxing the circuit-hyperplane $H$ of $M \backslash e$.

ii. Dually, when $f \in H, M \backslash f=M^{\prime} \backslash f$ and, unless $f$ is a loop of $M, M^{\prime} / f$ is obtained from $M / f$ by relaxing the circuit-hyperplane $X-\{f\}$ of $M / f$.

\subsection{Transversals}

Definition 2.14. Let $S$ be any set. Take a family of subsets $\mathcal{A}=\left(A_{1}, \ldots, A_{k}\right)$ of $S$. A transversal or system of distinct representatives of $\mathcal{A}$ is a subset $\left\{s_{1}, \ldots, s_{m}\right\}$ of $S$ such that $s_{i} \in A_{i}$ for all $i \in\{1, \ldots, m\}$ and $s_{1}, \ldots, s_{m}$ are distinct.

Definition 2.15. Let $S$ be any set. $X \subseteq S$ is a partial transversal of a family of subsets $\mathcal{A}=\left(A_{1}, \ldots, A_{j}\right)$ of $S$ if $X$ is a transversal of $\left(A_{1}, \ldots, A_{k}\right)$ for some $A_{1}, \ldots, A_{k} \subseteq S$.

Lemma 2.16. Let $\mathcal{A}=\left(A_{1}, \ldots, A_{m}\right)$ be a family of subsets of a set $S$. When $\mathcal{A}$ is a partition of $S$, the collection of partial transversals of $\mathcal{A}$ is the collection of independent sets of a matroid on $S$. This matroid is denoted by $M[\mathcal{A}]$. 
If a matroid $M$ is isomorphic to $M[\mathcal{A}]$ for some family of subsets $\mathcal{A}$, we say that $\mathrm{M}$ is a transversal matroid and that $\mathcal{A}$ is a presentation of $M$. Every transversal matroid is representable over all sufficiently large fields, as proved in [7, Proposition 11.2.16].

A transversal matroid can be represented by a bipartite graph. Let $\mathcal{A}=$ $\left(A_{1}, \ldots, A_{m}\right)$ be a family of subsets of $S$, and let $J=\{1, \ldots, m\}$. Construct the graph $G[\mathcal{A}]$ which has vertex set $S \cup J$ and edge set $\left\{x j \mid x \in A_{j}, j \in J\right\}$. Recall that a matching of a graph is a collection of edges such that no two share a common endpoint. A subset $X$ is a partial transversal of $\mathcal{A}$ if and only if there is a matching in $G[\mathcal{A}]$ in which every edge has an endpoint in $X$, i.e. $X$ is matched into $J$.

Definition 2.17. Take a matroid $M=(E, r)$ with independent sets $\mathcal{I}$. Let $\mathcal{J}=$ $\{I \in \mathcal{I}|| I \mid=r(M)\}$. The truncation of $M$ is a matroid $T(M)=(E, r)$ with independent sets $\mathcal{I}-\mathcal{J}$.

\subsection{Free extensions}

Definition 2.18. Take a matroid $M=(E, r)$. An element $e$ is freely placed in a flat $F$ of $M$ if, for any set $Z \subseteq E, e \in \operatorname{cl}(Z)$ implies $F \in \operatorname{cl}(Z)$.

Definition 2.19. Let $M=(E, r)$ be a matroid. Add an element $e \notin E$ freely to $E$. This gives a matroid $(E \cup\{e\}, r)$, which we call a free extension of $M$. 


\section{Chapter 3}

\section{Kinser Inequalities}

We will now introduce the Kinser inequalities, developed by Kinser in 2009 in [4]. An example of a matroid which exemplifies inequality $n$ for all $n \geq 4$ will be described, as the Vámos matroid exemplifies the Ingleton inequality. If a single circuit-hyperplane of this matroid is relaxed, it no longer satisfies the inequality. These matroids will be used in further results in this thesis. We will show that the class of matroids which satisfy Kinser inequality $n$ for all $n \geq 4$ is minor-closed. These classes are also closed under direct sums.

\subsection{Inequalities}

Definition 3.1. Let $M$ be a matroid, and let $X_{1}, \ldots, X_{n}$ be any collection of subsets of $E(M)$. Kinser inequality $n$, where $n \geq 4$, is

$$
\begin{aligned}
& \sum_{i=3}^{n} r\left(X_{i}\right)+r\left(X_{1} \cup X_{2}\right)+r\left(X_{1} \cup X_{3} \cup X_{n}\right)+\sum_{i=4}^{n} r\left(X_{2} \cup X_{i-1} \cup X_{i}\right) \\
& \quad \leq r\left(X_{1} \cup X_{3}\right)+r\left(X_{1} \cup X_{n}\right)+\sum_{i=3}^{n} r\left(X_{2} \cup X_{i}\right)+\sum_{i=4}^{n} r\left(X_{i-1} \cup X_{i}\right)
\end{aligned}
$$

We often use inequality $n$ to refer to Kinser inequality $n$. Note that inequality $n$ has $2 n-3$ terms on each side. 


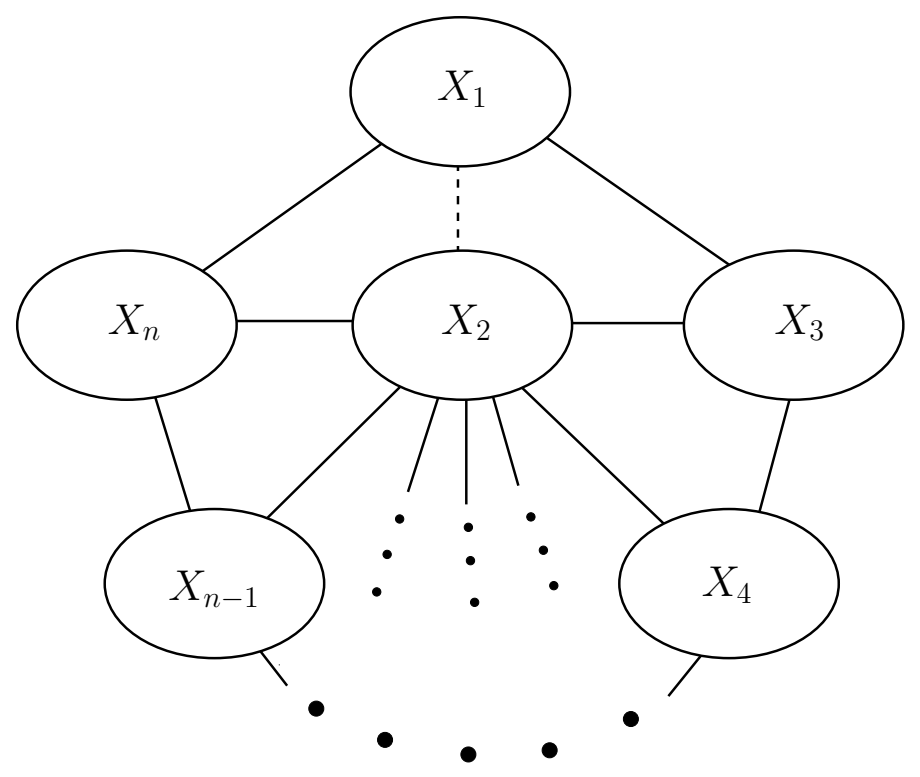

The above diagram gives a representation of Kinser inequality $n$. The ovals represent the $n$ subsets of $E(M)$, and each edge aside from the dotted one between $X_{1}$ and $X_{2}$ represents a term on the right-hand side of the inequality. On the left-hand side, we have the singleton sets starting from $X_{3}$, the triple $X_{1} \cup X_{3} \cup X_{n}$ at the very top, the dashed $X_{1} \cup X_{2}$ edge, and every triangle of edges involving $X_{2}$, excluding those using $X_{1}$.

When $n=4$, this yields the Ingleton inequality [3], which holds for any four subspaces $X_{1}, \ldots, X_{4}$ of a vector space:

$$
\begin{aligned}
& \operatorname{dim}\left(V_{3}\right)+\operatorname{dim}\left(V_{4}\right)+\operatorname{dim}\left(V_{1}+V_{2}\right)+\operatorname{dim}\left(V_{1}+V_{3}+V_{4}\right)+\operatorname{dim}\left(V_{2}+V_{3}+V_{4}\right) \\
\leq & \operatorname{dim}\left(V_{1}+V_{3}\right)+\operatorname{dim}\left(V_{1}+V_{4}\right)+\operatorname{dim}\left(V_{2}+V_{3}\right)+\operatorname{dim}\left(V_{2}+V_{4}\right)+\operatorname{dim}\left(V_{3}+V_{4}\right)
\end{aligned}
$$

As a representable matroid can be embedded inside a vector space, this inequality clearly holds for such matroids. In fact, in order for a matroid to be representable, it must satisfy each Kinser inequality for all choices of families $X_{1}, \ldots, X_{n}$.

Recall that if $X$ and $Y$ are subspaces of some vector space $\mathcal{V}$, then

$$
X+Y=\{\mathbf{x}+\mathbf{y} \mid \mathbf{x} \in X, \mathbf{y} \in Y\}
$$

is a subspace of $\mathcal{V}$ as well.

The following proof is adapted from that of [4, Theorem 1], which was stated in terms of an arrangement of $n$ subspaces. 
Lemma 3.2. A representable matroid $M$ satisfies each Kinser inequality.

Proof. Let $M$ be a matroid representable over an infinite field $\mathbb{K}$, and let $V_{1}, \ldots, V_{n}$ be subsets of $E(M)$. Embed $M$ in the projective geometry $P G(r-1, \mathbb{K})$ and replace each $V_{i}$ with its closure $\left\langle V_{i}\right\rangle$ in the projective geometry. Let $W=\left\langle V_{3}\right\rangle \cap \ldots \cap$ $\left\langle V_{n}\right\rangle$. Let $\left|\left\langle V_{i}\right\rangle\right|$ denote the dimension of $\left\langle V_{i}\right\rangle$. Using sub-modularity, we have that

$$
\begin{aligned}
\left|\langle W\rangle+\left\langle V_{1}\right\rangle\right|+\left|\langle W\rangle+\left\langle V_{2}\right\rangle\right| \geq & \left|\left(\langle W\rangle+\left\langle V_{1}\right\rangle\right) \cap\left(\langle W\rangle+\left\langle V_{2}\right\rangle\right)\right| \\
& +\left|\langle W\rangle+\left\langle V_{1}\right\rangle+\left\langle V_{2}\right\rangle\right| \\
\geq & \left|\langle W\rangle+\left(\left\langle V_{1}\right\rangle \cap\left\langle V_{2}\right\rangle\right)\right|+\left|\langle W\rangle+\left\langle V_{1}\right\rangle+\left\langle V_{2}\right\rangle\right| \\
\geq & |\langle W\rangle|+\left|\langle W\rangle+\left\langle V_{1}\right\rangle+\left\langle V_{2}\right\rangle\right|
\end{aligned}
$$

Rearranging this, we get that

$$
\left|\langle W\rangle+\left\langle V_{1}\right\rangle+\left\langle V_{2}\right\rangle\right|-\left|\langle W\rangle+\left\langle V_{1}\right\rangle\right| \leq\left|\langle W\rangle+\left\langle V_{2}\right\rangle\right|-|\langle W\rangle|
$$

We will give a bound on each side of this inequality.

Note that $\left|\langle W\rangle+\left\langle V_{1}\right\rangle+\left\langle V_{2}\right\rangle\right| \geq\left|\left\langle V_{1}\right\rangle+\left\langle V_{2}\right\rangle\right|$.

As $\langle W\rangle+\left\langle V_{1}\right\rangle \subseteq\left(\left\langle V_{1}\right\rangle+\left\langle V_{3}\right\rangle\right) \cap\left(\left\langle V_{1}\right\rangle+\left\langle V_{n}\right\rangle\right)$, we have by sub-modularity that

$$
\left|\langle W\rangle+\left\langle V_{1}\right\rangle\right| \leq\left|\left\langle V_{1}\right\rangle+\left\langle V_{3}\right\rangle\right|+\left|\left\langle V_{1}\right\rangle+\left\langle V_{n}\right\rangle\right|-\left|\left\langle V_{1}\right\rangle+\left\langle V_{3}\right\rangle+\left\langle V_{n}\right\rangle\right|
$$

This gives us a lower bound for the left-hand side of (3.2.1):

$$
\begin{aligned}
\left|\left\langle V_{1}\right\rangle+\left\langle V_{2}\right\rangle\right|-\left|\left\langle V_{1}\right\rangle+\left\langle V_{3}\right\rangle\right| & -\left|\left\langle V_{1}\right\rangle+\left\langle V_{n}\right\rangle\right|+\left|\left\langle V_{1}\right\rangle+\left\langle V_{3}\right\rangle+\left\langle V_{n}\right\rangle\right| \\
& \leq\left|\langle W\rangle+\left\langle V_{1}\right\rangle+\left\langle V_{2}\right\rangle\right|-\left|\langle W\rangle+\left\langle V_{1}\right\rangle\right|
\end{aligned}
$$

Now take the right-hand side. We have that

$$
\left|\langle W\rangle+\left\langle V_{2}\right\rangle\right|-|\langle W\rangle|=\left|\left\langle V_{2}\right\rangle\right|-\left|\left\langle V_{2}\right\rangle \cap\langle W\rangle\right|
$$

Note that $V_{2} \supseteq V_{2} \cap V_{3} \supseteq \ldots \supseteq V_{2} \cap \ldots \cap V_{n}=V_{2} \cap W$. This gives that

$$
\left|\left\langle V_{2}\right\rangle\right|-\left|\left\langle V_{2}\right\rangle \cap\langle W\rangle\right|=\sum_{i=3}^{n}\left(\left|\left\langle V_{2}\right\rangle \cap \ldots \cap\left\langle V_{i-1}\right\rangle\right|-\left|\left\langle V_{2}\right\rangle \cap \ldots \cap\left\langle V_{i}\right\rangle\right|\right)
$$

For each summand in 3.2 .2 , we give an upper bound: for $3 \leq i \leq n$, sub-modularity 
gives that

$$
\left|\left\langle V_{2}\right\rangle \cap \ldots \cap\left\langle V_{i-1}\right\rangle\right|-\left|\left\langle V_{2}\right\rangle \cap \ldots \cap\left\langle V_{i}\right\rangle\right|=\left|\left\langle V_{i}\right\rangle+\left(\left\langle V_{2}\right\rangle \cap \ldots \cap\left\langle V_{i-1}\right)\right\rangle\right|-\left|\left\langle V_{i}\right\rangle\right|
$$

As $\left\langle V_{i}\right\rangle+\left(\left\langle V_{2}\right\rangle \cap \ldots \cap\left\langle V_{i-1}\right\rangle\right) \subseteq\left(\left\langle V_{i}\right\rangle+\left\langle V_{2}\right\rangle\right) \cap\left(\left\langle V_{i}\right\rangle+\left\langle V_{i-1}\right\rangle\right)$, we have

$$
\begin{aligned}
\left|\left\langle V_{i}\right\rangle+\left(\left\langle V_{2}\right\rangle \cap \ldots \cap\left\langle V_{i-1}\right\rangle\right)\right|-\left|\left\langle V_{i}\right\rangle\right| \leq\left|\left(\left\langle V_{i}\right\rangle+\left\langle V_{2}\right\rangle\right) \cap\left(\left\langle V_{i}\right\rangle+\left\langle V_{i-1}\right)\right\rangle\right|-\left|\left\langle V_{i}\right\rangle\right| \\
=\left|\left\langle V_{i}\right\rangle+\left\langle V_{2}\right\rangle\right|+\left|\left\langle V_{i}\right\rangle+\left\langle V_{i-1}\right\rangle\right| \\
-\left|\left\langle V_{2}\right\rangle+\left\langle V_{i-1}\right\rangle+\left\langle V_{i}\right\rangle\right|-\left|\left\langle V_{i}\right\rangle\right|
\end{aligned}
$$

Note that when $i=3$ this simplifies to

$$
\begin{aligned}
\left|\left\langle V_{3}\right\rangle+\left\langle V_{2}\right\rangle\right|-\left|\left\langle V_{3}\right\rangle\right| & \leq\left|\left\langle V_{3}\right\rangle+\left\langle V_{2}\right\rangle\right|+\left|\left\langle V_{3}\right\rangle+\left\langle V_{2}\right\rangle\right|-\left|\left\langle V_{2}\right\rangle+\left\langle V_{3}\right\rangle\right|-\left|\left\langle V_{3}\right\rangle\right| \\
& =\left|\left\langle V_{2}\right\rangle+\left\langle V_{3}\right\rangle\right|-\left|\left\langle V_{3}\right\rangle\right|
\end{aligned}
$$

Plugging this into 3.2.2 then 3.2.1 gives, after rearranging,

$$
\begin{array}{r}
\sum_{i=3}^{n}\left|\left\langle V_{i}\right\rangle\right|+\left|\left\langle V_{1}\right\rangle+\left\langle V_{2}\right\rangle\right|+\left|\left\langle V_{1}\right\rangle+\left\langle V_{3}\right\rangle+\left\langle V_{n}\right\rangle\right|+\sum_{i=4}^{n}\left|\left\langle V_{2}\right\rangle+\left\langle V_{i-1}\right\rangle+\left\langle X_{i}\right\rangle\right| \\
\quad \leq\left|\left\langle V_{1}\right\rangle+\left\langle V_{3}\right\rangle\right|+\left|\left\langle V_{1}\right\rangle+\left\langle V_{n}\right\rangle\right|+\sum_{i=3}^{n}\left|\left\langle V_{2}\right\rangle+\left\langle V_{i}\right\rangle\right|+\sum_{i=4}^{n}\left|\left\langle V_{i-1}\right\rangle+\left\langle V_{i}\right\rangle\right|
\end{array}
$$

Note that $\left|\left\langle V_{i}\right\rangle\right|=r\left(V_{i}\right)$. In order to show that inequality $n$ holds, we must show that $\left|\left\langle V_{i}\right\rangle+\left\langle V_{j}\right\rangle\right|=r\left(V_{i} \cup V_{j}\right)$. We have that

$$
r\left(V_{i} \cup V_{j}\right)=\left|\left\langle V_{i} \cup V_{j}\right\rangle\right|
$$

We will show that this is equal to $\left|\left\langle V_{i}\right\rangle+\left\langle V_{j}\right\rangle\right|$.

Let $x \in\left\langle V_{i}\right\rangle+\left\langle V_{j}\right\rangle$. This means that $x=x_{1}+x_{2}$ where $x_{1} \in\left\langle V_{i}\right\rangle$ and $x_{2} \in\left\langle V_{j}\right\rangle$. We have that $x_{1} \in\left\langle V_{i} \cup V_{j}\right\rangle$ and $x_{2} \in\left\langle V_{i} \cup V_{j}\right\rangle$, so $x \in\left\langle V_{i} \cup V_{j}\right\rangle$. Now take $x \in$ $\left\langle V_{i} \cup V_{j}\right\rangle$. We can write $x$ as a linear combination of elements $S_{i}$ from $V_{i}$ and elements $S_{j}$ from $V_{j}$. We have that $S_{i} \subseteq\left\langle V_{i}\right\rangle$ and that $S_{j} \subseteq\left\langle V_{j}\right\rangle$, so $x \in\left\langle V_{i}\right\rangle+\left\langle V_{j}\right\rangle$. Thus $\left|\left\langle V_{i} \cup V_{j}\right\rangle\right|=\left|\left\langle V_{i}\right\rangle+\left\langle V_{j}\right\rangle\right|$. We can therefore replace every term $\left|\left\langle V_{i}\right\rangle+\left\langle V_{j}\right\rangle\right|$ with $r\left(V_{i} \cup V_{j}\right)$. Similarly, $\left|\left\langle V_{i} \cup V_{j} \cup V_{k}\right\rangle\right|=\left|\left\langle V_{i}\right\rangle+\left\langle V_{j}\right\rangle+\left\langle V_{k}\right\rangle\right|$. Making all such replacements yields inequality $n$. 
We say that a bad family for a matroid $M$, relative to $n$, is a family of subsets $X_{1}, \ldots, X_{n}$ which does not satisfy Kinser inequality $n$.

We can also represent an inequality as applied to a specific matroid with a graph. Let $X_{1}, \ldots, X_{n}$ be a family of subsets of a matroid $M$. Take a graph $G$ on vertices $V=\left\{X_{1}, \ldots, X_{n}\right\}$ with adjacency relation $a$ such that

$$
a\left(X_{i}\right)=\left\{X_{j} \mid X_{i} \cup X_{j} \text { is a term on the right-hand side of inequality } n\right\} .
$$

In other words, two vertices are joined by an edge if the union of the two vertices is a term in inequality $n$. Recall that when $G[V, E]$ is any graph with vertex set $V$ and edge set $E$, an induced subgraph $G\left[E^{\prime}\right]$, has edge set $E^{\prime}$ and vertex set equal to the vertices incident with edges in $E^{\prime}$. We will use this construction to show that certain subgraph structures cannot exist, when attempting to find a bad family in a matroid.

Definition 3.3. Kinser class $n$, denoted by $\mathcal{K}_{n}$, is the set of matroids which satisfy Kinser inequality $n$ for all families of subsets $X_{1}, \ldots, X_{n}$ of the ground set. We define $\mathcal{K}_{\infty}=\bigcap_{i \geq 4} \mathcal{K}_{i}$.

A matroid $M$ has a bad family relative to $n$ if and only if $M \notin \mathcal{K}_{n}$.

Definition 3.4. The dual Kinser class $n$ is $\mathcal{K}_{n}^{*}=\left\{M^{*} \mid M \in \mathcal{K}_{n}\right\}$

\subsection{Kinser matroids}

Next we will construct a class of matroids called Kinser matroids, relating to the Kinser inequalities as the Vámos matroid [7, Figure 2.4] relates to the Ingleton inequality. In fact, the Vámos matroid is obtained from the fourth Kinser matroid by relaxing a circuit-hyperplane. The rank- $r$ Kinser matroid, for $r \geq 4$, is denoted by $\operatorname{Kin}(r)$, and has a ground set of size $r^{2}-3 r+4$.

First, we will define a rank $r+1$ transversal matroid, $M_{r+1}$. Let $\mathcal{A}=$ $\left(A_{1}, A_{3}, \ldots, A_{r}, A, A^{\prime}\right)$. Also let $V_{1}, \ldots, V_{r}$ be pairwise disjoint sets such that

$$
\left|V_{1}\right|=\left|V_{3}\right|=\cdots=\left|V_{r}\right|=r-2
$$

and $V_{2}=\{e, f\}$. The ground set of $M_{r+1}$ is $V_{1} \cup \cdots \cup V_{r}$. Let $A=E\left(M_{r+1}\right)$ and let 
$A^{\prime}=V_{2}$. Let

$$
\begin{aligned}
& A_{1}=\left(V_{1} \cup V_{3} \cup \cdots \cup V_{r}\right)-\left(V_{1} \cup V_{r}\right) \\
& A_{3}=\left(V_{1} \cup V_{3} \cup \cdots \cup V_{r}\right)-\left(V_{1} \cup V_{3}\right)
\end{aligned}
$$

For $i \in\{4, \ldots, r\}$, let

$$
A_{i}=\left(V_{1} \cup V_{3} \cup \cdots \cup V_{r}\right)-\left(V_{i-1} \cup V_{i}\right)
$$

Note $\{e, f\}$ is a series pair in $M_{r+1}$.

$M_{r+1}$ is the transversal matroid $M[\mathcal{A}]$. Define $\operatorname{Kin}(r)$ to be the truncation of $M_{r+1}$.

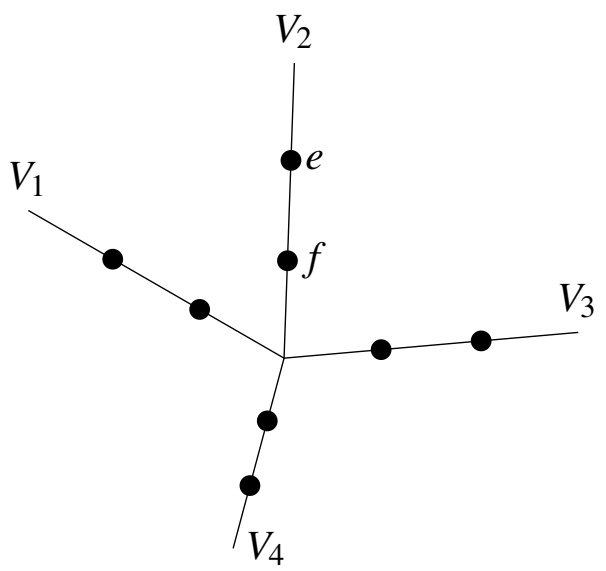

Figure 3.1: $\operatorname{Kin}(4)$

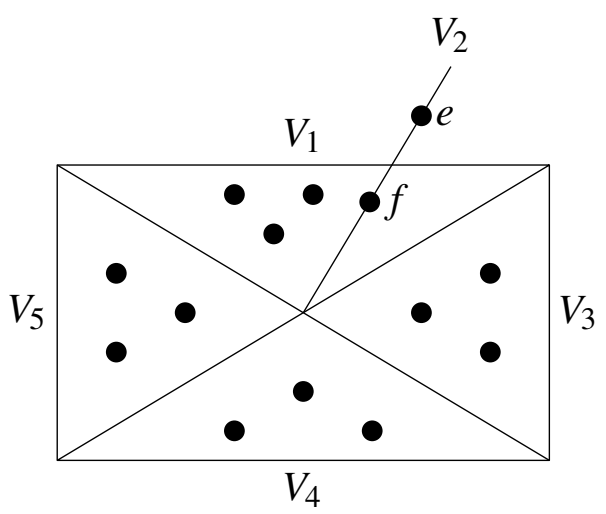

Figure 3.2: $\operatorname{Kin}(5)$ 
The following result is Proposition 4.3 of [5]

Lemma 3.5. Let $\mathbb{K}$ be an infinite field. Then $\operatorname{Kin}(r)$ is $\mathbb{K}$-representable for any $r \geq 4$.

As $M_{r+1}$ is a transversal matroid, it is representable over every infinite field by [7, Proposition 11.2.16]. We obtain $\operatorname{Kin}(r)$ by truncating $M_{r+1}$. This is equivalent to freely adding an element to the ground set of $M_{r+1}$ and then contracting it. As the class of representable matroids is closed under free extensions, $\operatorname{Kin}(r)$ is also representable.

The following result is proved in [5, Proposition 4.4].

Lemma 3.6. Let $r \geq 4$ be an integer. Then $V_{2} \cup V_{i}$ is a circuit-hyperplane of $\operatorname{Kin}(r)$ for any $i \in\{1,3, \ldots, r\}$.

Define $\operatorname{Kin}(r)^{-}$to be the matroid obtained from $\operatorname{Kin}(r)$ by relaxing the circuithyperplane $V_{1} \cup V_{2}$. Also define $\operatorname{Kin}(r)_{i}^{=}$to be the matroid obtained from $\operatorname{Kin}(r)$ by relaxing the circuit-hyperplanes $V_{1} \cup V_{2}$ and $V_{2} \cup V_{i}$, for some $i \in\{3, \ldots, r\}$.

The next two results are Proposition 4.5 and Lemma 4.6 of [5].

Lemma 3.7. Let $r \geq 4$. The matroid $\operatorname{Kin}(r)^{-}$is not in $\mathcal{K}_{r}$, and is therefore not representable over any field.

The family of subsets $V_{1}, \ldots, V_{n}$ in $\operatorname{Kin}(r)^{-}$is a bad family relative to $r$, as will be proved in Lemma 4.3 .

Lemma 3.8. Let $r \geq 4$ and let $\mathbb{K}$ be an infinite field. The matroid $\operatorname{Kin}(r)_{i}=$ is $\mathbb{K}$-representable.

\subsection{Kinser classes}

Lemma 3.9. $\mathcal{K}_{n}$ is minor-closed for all $n \geq 4$.

Proof. Take some $M \in \mathcal{K}_{n}$ and $e \in E(M)$ such that $M / e \notin \mathcal{K}_{n}$. Assume $e$ is not a loop. Assume $X_{1}, \ldots, X_{n}$ is a bad family in $M / e$ and let $X_{i}^{\prime}=X_{i} \cup\{e\}$ for all 
$i$. Recall that $r_{M / x}(X)=r_{M}(X \cup x)-r_{M}(x)$. Thus $r_{M / e}\left(X_{i}\right)=r_{M}\left(X_{i} \cup e\right)-r_{M}(e)$. When $e$ is not a loop, we have that $r_{M}\left(X_{i}^{\prime}\right)=r_{M / e}\left(X_{i}\right)+1$ for all $i$, and

$$
r_{M}\left(X_{i}^{\prime} \cup X_{j}^{\prime} \cup X_{k}^{\prime}\right)=r_{M / e}\left(X_{i} \cup X_{j} \cup X_{k}\right)+1
$$

Now evaluate inequality $n$ for $X_{1}^{\prime}, \ldots, X_{n}^{\prime}$ in $M$.

$$
\begin{aligned}
\sum_{i=3}^{n} r_{M}\left(X_{i}^{\prime}\right) & +r_{M}\left(X_{1}^{\prime} \cup X_{2}^{\prime}\right)+r_{M}\left(X_{1}^{\prime} \cup X_{3}^{\prime} \cup X_{n}^{\prime}\right)+\sum_{i=4}^{n} r_{M}\left(X_{2}^{\prime} \cup X_{i-1}^{\prime} \cup X_{i}^{\prime}\right) \\
\leq & r_{M}\left(X_{1}^{\prime} \cup X_{3}^{\prime}\right)+r_{M}\left(X_{1}^{\prime} \cup X_{n}^{\prime}\right)+\sum_{i=3}^{n} r_{M}\left(X_{2}^{\prime} \cup X_{i}^{\prime}\right)+\sum_{i=4}^{n} r_{M}\left(X_{i-1}^{\prime} \cup X_{i}^{\prime}\right)
\end{aligned}
$$

Using the rank equalities calculated above, this is equivalent to

$$
\begin{aligned}
& \sum_{i=3}^{n}\left(r_{M / x}\left(X_{i}\right)+1\right)+ r_{M / x}\left(X_{1} \cup X_{2}\right)+1 \\
&+r_{M / x}\left(X_{1} \cup X_{3} \cup X_{n}\right)+1+\sum_{i=4}^{n}\left(r_{M / x}\left(X_{2} \cup X_{i-1} \cup X_{i}\right)+1\right) \\
& \leq r_{M / x}\left(X_{1} \cup X_{3}\right)+1+r_{M / x}\left(X_{1} \cup X_{n}\right)+1 \\
&+\sum_{i=3}^{n}\left(r_{M / x}\left(X_{2} \cup X_{i}\right)+1\right)+\sum_{i=4}^{n}\left(r_{M / x}\left(X_{i-1} \cup X_{i}\right)+1\right)
\end{aligned}
$$

All the constant terms cancel out, leaving inequality $n$ as applied to $X_{1}, \ldots, X_{n}$ in $M / e$, contradicting $X_{1}, \ldots, X_{n}$ being a bad family in $M / e$. Thus there is no $e$ such that $M / e \notin \mathcal{K}_{n}$.

Now consider $M \backslash e$. Assume that $M \backslash e$ has a bad family $X_{1}, \ldots, X_{n}$. These subsets are also subsets of $M$ and their rank is unchanged, so they must form a bad family in $M$ as well, contradicting $M \in \mathcal{K}_{n}$.

Lemma 3.10. Suppose $X_{1}, \ldots, X_{n}$ is a bad family for Kinser inequality $n$. Then there exists a bad family $Y_{1}, \ldots, Y_{n}$ such that each $Y_{i}$ is a basis of $X_{i}$.

Proof. Take some matroid $M$ with bad family $X_{1}, \ldots, X_{n}$. For each $X_{j}$ in the bad family, let $I_{j}$ be a basis of $M \mid X_{j}$. We will show that we can replace each set $X_{j}$ with its basis $I_{j}$. We have that $r\left(X_{j}\right)=r\left(I_{j}\right)$. Now consider $r\left(X_{j} \cup X_{k}\right)$. Clearly $r\left(I_{j} \cup I_{k}\right) \leq r\left(X_{j} \cup X_{k}\right)$. If $x \in X_{j} \cup X_{k}$, then $x$ is either in $X_{j}$ or it is in $X_{j}$, so $x \in \mathrm{cl}_{M}\left(I_{j}\right)$ or $x \in \mathrm{cl}_{M}\left(I_{k}\right)$. In either case, $x \in \mathrm{cl}_{M}\left(I_{j} \cup I_{k}\right)$, so $X_{j} \cup X_{k} \subseteq \mathrm{cl}_{M}\left(I_{j} \cup I_{k}\right)$. 
Thus

$$
\begin{aligned}
r\left(X_{j} \cup X_{k}\right) & \leq r\left(\mathrm{cl}_{M}\left(I_{j} \cup I_{k}\right)\right) \\
& =r\left(I_{j} \cup I_{k}\right)
\end{aligned}
$$

Thus $r\left(I_{j} \cup I_{k}\right) \leq r\left(X_{j} \cup X_{k}\right) \leq r\left(I_{j} \cup I_{k}\right)$, so $r\left(X_{j} \cup X_{k}\right)=r\left(I_{j} \cup I_{k}\right)$. Similarly, $r\left(X_{j} \cup X_{k} \cup X_{l}\right)=r\left(I_{j} \cup I_{k} \cup I_{l}\right)$. Thus $I_{1}, \ldots, I_{n}$ is a bad family for Kinser inequality $n$.

Lemma 3.11. Suppose $X_{1}, \ldots, X_{n}$ is a bad family for Kinser inequality $n$. Then there exists a bad family $Y_{1}, \ldots, Y_{n}$ such that each $Y_{i}$ is a flat.

Proof. Recall that a flat is a set $X$ such that $\operatorname{cl}(X)=X$. We simply replace each $X_{i}$ with $\operatorname{cl}\left(X_{i}\right)$. First note that $r\left(\operatorname{cl}\left(X_{i}\right)\right)=r\left(X_{i}\right)$. Now consider $r\left(X_{i} \cup X_{j}\right)=r\left(\operatorname{cl}\left(X_{i} \cup\right.\right.$ $\left.X_{j}\right)$ ). We need to show that this is equal to $r\left(\operatorname{cl}\left(X_{i}\right) \cup \operatorname{cl}\left(X_{j}\right)\right)$. As $X_{i} \subseteq \operatorname{cl}\left(X_{i}\right)$, we must have that

$$
r\left(X_{i} \cup X_{j}\right) \leq r\left(\operatorname{cl}\left(X_{i}\right) \cup \operatorname{cl}\left(X_{j}\right)\right) .
$$

Now note that if $e \in \operatorname{cl}\left(X_{i}\right)$, then $e \in \operatorname{cl}\left(X_{i} \cup X_{j}\right)$. This implies that $\operatorname{cl}\left(X_{i}\right) \subseteq \operatorname{cl}\left(X_{i} \cup\right.$ $\left.X_{j}\right)$ Likewise, every element in the closure of $X_{j}$ is also in the closure of $X_{i} \cup X_{j}$, so $\operatorname{cl}\left(X_{j}\right) \subseteq \operatorname{cl}\left(X_{i} \cup X_{j}\right)$. We thus have that $\operatorname{cl}\left(X_{i}\right) \cup \operatorname{cl}\left(X_{j}\right) \subseteq \operatorname{cl}\left(X_{i} \cup X_{j}\right)$, so

$$
\begin{aligned}
r\left(\operatorname{cl}\left(X_{i}\right) \cup \operatorname{cl}\left(X_{j}\right)\right) & \leq r\left(\operatorname{cl}\left(X_{i} \cup X_{j}\right)\right) \\
& =r\left(X_{i} \cup X_{j}\right)
\end{aligned}
$$

We thus have that

$$
r\left(\operatorname{cl}\left(X_{i}\right) \cup \operatorname{cl}\left(X_{j}\right)\right) \leq r\left(X_{i} \cup X_{j}\right) \leq r\left(\operatorname{cl}\left(X_{i}\right) \cup \operatorname{cl}\left(X_{j}\right)\right)
$$

so $r\left(X_{i} \cup X_{j}\right)=r\left(\operatorname{cl}\left(X_{i}\right) \cup \operatorname{cl}\left(X_{j}\right)\right)$, and so $\operatorname{cl}\left(X_{i}\right), \ldots, \operatorname{cl}\left(X_{n}\right)$ is a bad family for inequality $n$ as well.

\subsection{Sums}

Definition 3.12. Take $M=(E, r)$ and $M^{\prime}=\left(E^{\prime}, r^{\prime}\right)$ where $E \cap E^{\prime}=\varnothing$. The direct sum of these matroids is denoted by $M \oplus M^{\prime}$, and has ground set $E \cup E^{\prime}$ and rank of $X \subseteq E \cup E^{\prime}$ equal to $r(X \cap E)+r^{\prime}\left(X \cap E^{\prime}\right)$. 
Lemma 3.13. $\mathcal{K}_{n}$ is closed under direct sum for all $n$.

Proof. Take two matroid $M=(E, r)$ and $M^{\prime}=\left(E^{\prime}, r^{\prime}\right)$ which are contained in $\mathcal{K}_{n}$. Take the direct sum $M \oplus M^{\prime}$. We wish to show that for any family $X_{1}, \ldots, X_{n}$ of $E \cup E^{\prime}$ the following inequality holds:

$$
\begin{gathered}
\sum_{i=3}^{n} r_{M \oplus M^{\prime}}\left(X_{i}\right)+r_{M \oplus M^{\prime}}\left(X_{1} \cup X_{2}\right)+r_{M \oplus M^{\prime}}\left(X_{1} \cup X_{3} \cup X_{n}\right)+\sum_{i=4}^{n} r_{M \oplus M^{\prime}}\left(X_{2} \cup X_{i-1} \cup X_{i}\right) \\
\quad \leq r_{M \oplus M^{\prime}}\left(X_{1} \cup X_{3}\right)+r_{M \oplus M^{\prime}}\left(X_{1} \cup X_{n}\right)+\sum_{i=3}^{n} r_{M \oplus M^{\prime}}\left(X_{2} \cup X_{i}\right)+\sum_{i=4}^{n} r_{M \oplus M^{\prime}}\left(X_{i-1} \cup X_{i}\right)
\end{gathered}
$$

This is equivalent to

$$
\begin{aligned}
& \sum_{i=3}^{n} r\left(X_{i} \cap E\right)+r\left(\left(X_{1} \cup X_{2}\right) \cap E\right)+r\left(\left(X_{1} \cup X_{3} \cup X_{n}\right) \cap E\right) \\
& \quad+\sum_{i=4}^{n} r\left(\left(X_{2} \cup X_{i-1} \cup X_{i}\right) \cap E\right)+\sum_{i=3}^{n} r^{\prime}\left(X_{i} \cap E^{\prime}\right)+r^{\prime}\left(\left(X_{1} \cup X_{2}\right) \cap E^{\prime}\right) \\
& \quad+r^{\prime}\left(\left(X_{1} \cup X_{3} \cup X_{n}\right) \cap E^{\prime}\right)+\sum_{i=4}^{n} r^{\prime}\left(\left(X_{2} \cup X_{i-1} \cup X_{i}\right) \cap E^{\prime}\right) \\
& \leq r\left(\left(X_{1} \cup X_{3}\right) \cap E\right)+r\left(\left(X_{1} \cup X_{n}\right) \cap E\right)+\sum_{i=3}^{n} r\left(\left(X_{2} \cup X_{i}\right) \cap E\right) \\
& \quad+\sum_{i=4}^{n} r\left(\left(X_{i-1} \cup X_{i}\right) \cap E\right)+r^{\prime}\left(\left(X_{1} \cup X_{3}\right) \cap E^{\prime}\right)+r^{\prime}\left(\left(X_{1} \cup X_{n}\right) \cap E^{\prime}\right) \\
& +\sum_{i=3}^{n} r^{\prime}\left(\left(X_{2} \cup X_{i}\right) \cap E^{\prime}\right)+\sum_{i=4}^{n} r^{\prime}\left(\left(X_{i-1} \cup X_{i}\right) \cap E^{\prime}\right)
\end{aligned}
$$

As $M \in \mathcal{K}_{n}$, we have that

$$
\begin{aligned}
& \sum_{i=3}^{n} r\left(X_{i} \cap E\right)+r\left(\left(X_{1} \cup X_{2}\right) \cap E\right) \\
& \quad+r\left(\left(X_{1} \cup X_{3} \cup X_{n}\right) \cap E\right)+\sum_{i=4}^{n} r\left(\left(X_{2} \cup X_{i-1} \cup X_{i}\right) \cap E\right) \\
& \leq r\left(\left(X_{1} \cup X_{3}\right) \cap E\right)+r\left(\left(X_{1} \cup X_{n}\right) \cap E\right) \\
& \quad+\sum_{i=3}^{n} r\left(\left(X_{2} \cup X_{i}\right) \cap E\right)+\sum_{i=4}^{n} r\left(\left(X_{i-1} \cup X_{i}\right) \cap E\right)
\end{aligned}
$$


As $M^{\prime} \in \mathcal{K}_{n}$, we have that

$$
\begin{aligned}
& r^{\prime}\left(X_{i} \cap E^{\prime}\right)+r^{\prime}\left(\left(X_{1} \cup X_{2}\right) \cap E^{\prime}\right) \\
& \quad+r^{\prime}\left(\left(X_{1} \cup X_{3} \cup X_{n}\right) \cap E^{\prime}\right)+\sum_{i=4}^{n} r^{\prime}\left(\left(X_{2} \cup X_{i-1} \cup X_{i}\right) \cap E^{\prime}\right) \\
& \leq r^{\prime}\left(\left(X_{1} \cup X_{3}\right) \cap E^{\prime}\right)+r^{\prime}\left(\left(X_{1} \cup X_{n}\right) \cap E^{\prime}\right) \\
& \quad+\sum_{i=3}^{n} r^{\prime}\left(\left(X_{2} \cup X_{i}\right) \cap E^{\prime}\right)+\sum_{i=4}^{n} r^{\prime}\left(\left(X_{i-1} \cup X_{i}\right) \cap E^{\prime}\right)
\end{aligned}
$$

The values of the terms on the left of inequality 3.13.1) are thus bounded by the terms on the right-hand side, and so the inequality holds. 


\section{Chapter 4}

\section{Kinser Hierarchy}

In this chapter we will investigate how the Kinser classes interact with each other. We will first show that representable matroids are properly contained inside every Kinser class. Next we will show that the classes form a descending chain, and show that the relaxed Kinser matroid of rank $n$ is contained inside $\mathcal{K}_{n-1}$ but not $\mathcal{K}_{n}$. Next we will consider the issue of duality, and prove that the class of matroids which satisfy Kinser inequality 4 is dual closed. The class of matroids which satisfy Kinser inequality 5 is, in contrast, not dual closed. The proof of this is given in the next chapter.

Lemma 4.1. The class of representable matroids is properly contained in $\mathcal{K}_{\infty}$

Proof. Note that as a consequence of Lemma 3.2, the class of representable matroids are contained inside every Kinser class, and so is a subset of $\mathcal{K}_{\infty}$. We will now show the class of representable matroids forms a proper subset of $\mathcal{K}_{\infty}$. Recall that $\mathcal{K}_{n}$ is closed under direct sum for all $n$. Take two matroids in $\mathcal{K}_{\infty}$. As these matroids are in the intersection of every Kinser class, their direct sum is also contained in every Kinser class, and so contained in $\mathcal{K}_{\infty}$. Thus $\mathcal{K}_{\infty}$ is also closed under direct sums.

Define $F_{7}$ to be the matroid represented over GF(2) by

$$
\left(\begin{array}{lllllll}
1 & 0 & 0 & 1 & 1 & 0 & 1 \\
0 & 1 & 0 & 1 & 0 & 1 & 1 \\
0 & 0 & 1 & 0 & 1 & 1 & 1
\end{array}\right)
$$

Define $F_{7}^{-}$to be the matroid represented by the same matrix, but over $\operatorname{GF}(3)$. 
By [7, Proposition 6.4.8], $F_{7}$ can be represented over a field only if the field has characteristic 2 , while $F_{7}^{-}$can be represented over a field only if the field has characteristic different from 2. Therefore $F_{7} \oplus F_{7}^{-}$is not representable. However, it is contained in $K_{\infty}$, since $F_{7}$ and $F_{7}^{-}$are both representable, and hence in $\mathcal{K}_{\infty}$. $\square$

\section{Lemma 4.2. $\mathcal{K}_{n} \supseteq \mathcal{K}_{n+1}$.}

Proof. Assume inequality $n+1$ holds for the matroid $M$. Let $X_{1}, \ldots, X_{n}$ be arbitrary subsets of $E(M)$. We show inequality $n$ holds for $X_{1}, \ldots, X_{n}$. Let $X_{n+1}=X_{n}$. We have that

$$
\begin{aligned}
& \sum_{i=3}^{n+1} r\left(X_{i}\right)+r\left(X_{1} \cup X_{2}\right)+r\left(X_{1} \cup X_{3} \cup X_{n+1}\right)+\sum_{i=4}^{n+1} r\left(X_{2} \cup X_{i-1} \cup X_{i}\right) \\
& \quad \leq r\left(X_{1} \cup X_{3}\right)+r\left(X_{1} \cup X_{n+1}\right)+\sum_{i=3}^{n+1} r\left(X_{2} \cup X_{i}\right)+\sum_{i=4}^{n+1} r\left(X_{i-1} \cup X_{i}\right)
\end{aligned}
$$

Bringing out the last term of each sum,

$$
\begin{aligned}
& \sum_{i=3}^{n} r\left(X_{i}\right)+r\left(X_{n+1}\right)+r\left(X_{1} \cup X_{2}\right)+r\left(X_{1} \cup X_{3} \cup X_{n}\right) \\
& \quad+\sum_{i=4}^{n} r\left(X_{2} \cup X_{i-1} \cup X_{i}\right)+r\left(X_{2} \cup X_{n+1}\right) \\
& \leq r\left(X_{1} \cup X_{3}\right)+r\left(X_{1} \cup X_{n}\right)+\sum_{i=3}^{n} r\left(X_{2} \cup X_{i}\right) \\
& \quad+r\left(X_{2} \cup X_{n+1}\right)+\sum_{i=4}^{n} r\left(X_{i-1} \cup X_{i}\right)+r\left(X_{n} \cup X_{n+1}\right)
\end{aligned}
$$

Now bringing these terms to the start of each side of the inequality and using $X_{n+1}=X_{n}$, we have that this is the same as

$$
\begin{aligned}
& r\left(X_{n}\right)+r\left(X_{2} \cup X_{n}\right)+\sum_{i=3}^{n} r\left(X_{i}\right)+r\left(X_{1} \cup X_{2}\right) \\
&+r\left(X_{1} \cup X_{3} \cup X_{n}\right)+\sum_{i=4}^{n} r\left(X_{2} \cup X_{i-1} \cup X_{i}\right) \\
& \leq r\left(X_{2} \cup X_{n}\right)+r\left(X_{n}\right)+r\left(X_{1} \cup X_{3}\right)+r\left(X_{1} \cup X_{n}\right) \\
&+\sum_{i=3}^{n} r\left(X_{2} \cup X_{i}\right)+\sum_{i=4}^{n} r\left(X_{i-1} \cup X_{i}\right)
\end{aligned}
$$

The two terms at the start of each side of the inequality cancel out, leaving in- 
equality $n$ :

$$
\begin{aligned}
& \sum_{i=3}^{n} r\left(X_{i}\right)+r\left(X_{1} \cup X_{2}\right)+r\left(X_{1} \cup X_{3} \cup X_{n}\right)+\sum_{i=4}^{n} r\left(X_{2} \cup X_{i-1} \cup X_{i}\right) \\
& \quad \leq r\left(X_{1} \cup X_{3}\right)+r\left(X_{1} \cup X_{n}\right)+\sum_{i=3}^{n} r\left(X_{2} \cup X_{i}\right)+\sum_{i=4}^{n} r\left(X_{i-1} \cup X_{i}\right) .
\end{aligned}
$$

We now have the following diagram of the Kinser hierarchy.

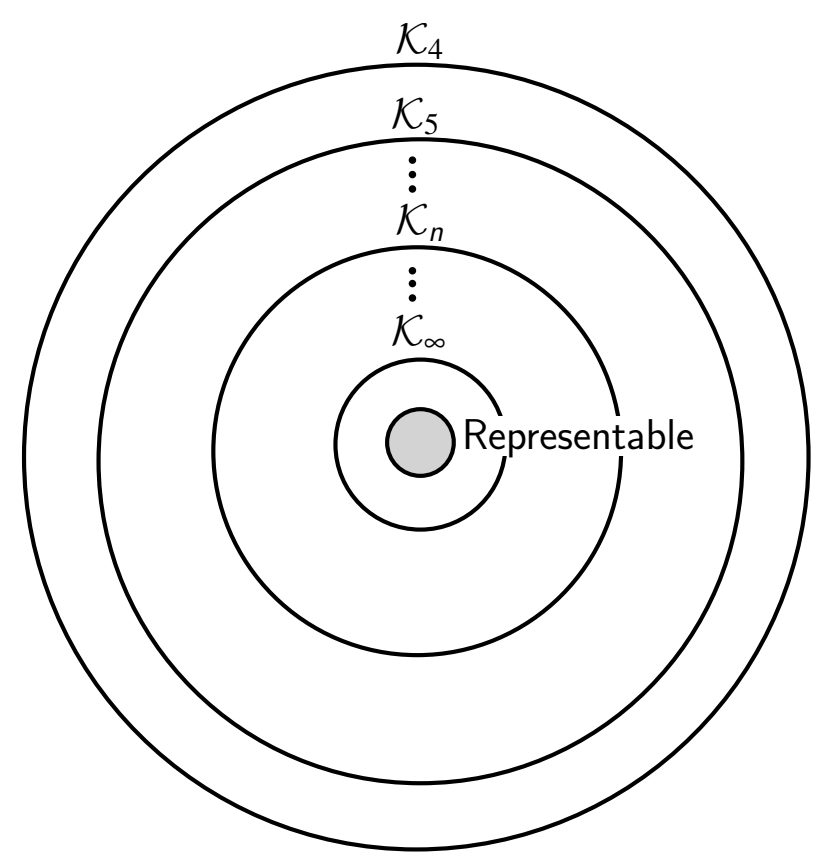

Figure 4.1: Kinser classes

Next, we will give an example of a matroid which lies in the gap between two of these classes.

Lemma 4.3. For $n \geq 5, \operatorname{Kin}(n)^{-} \in \mathcal{K}_{n-1}-\mathcal{K}_{n}$.

Proof. Take $\operatorname{Kin}(n)^{-}$. We will first show that $V_{1}, \ldots, V_{n}$ as in the definition of $\operatorname{Kin}(n)$ forms a bad family for inequality $n$ - that is,

$$
\begin{gathered}
\sum_{i=3}^{n} r\left(V_{i}\right)+r\left(V_{1} \cup V_{2}\right)+r\left(V_{1} \cup V_{3} \cup V_{n}\right)+\sum_{i=4}^{n} r\left(V_{2} \cup V_{i-1} \cup V_{i}\right) \\
\not \leq r\left(V_{1} \cup V_{3}\right)+r\left(V_{1} \cup V_{n}\right)+\sum_{i=3}^{n} r\left(V_{2} \cup V_{i}\right)+\sum_{i=4}^{n} r\left(V_{i-1} \cup V_{i}\right)
\end{gathered}
$$


We sketch the proof of [5, Proposition 4.5].

Recall that $V_{1} \cup V_{2}$ is a relaxed circuit-hyperplane, while $V_{2} \cup V_{i}$ is a circuithyperplane for all $i \in\{3, \ldots, n\}$. Also, $V_{i} \cup V_{i+1}$ is a hyperplane for all $i \geq 4$, as is $V_{1} \cup V_{3}$, while $V_{i} \cup V_{k}$ is spanning for inconsecutive $i, k$. Each $V_{i}$ is independent, while the union of any three $V_{i}$ is spanning. Substituting these results into inequality $n$ gives

$$
\begin{aligned}
& \sum_{i=3}^{n}(n-2)+n+n+\sum_{i=4}^{n} n \quad \not \leq \quad(n-1)+n(n-1) \\
& +\sum_{i=3}^{n}(n-1)+\sum_{i=4}^{n}(n-1) \\
& \Leftrightarrow(n-2)(n-2)+2 n+(n-3) n \quad \not \quad(2 n-3)(n-1) \\
& \Leftrightarrow \quad 2 n^{2}-5 n+4 \quad \not \quad 2 n^{2}-5 n+3
\end{aligned}
$$

Thus $\operatorname{Kin}(n)^{-} \notin \mathcal{K}_{n}$.

Assume $\operatorname{Kin}(n)^{-}$has a family $X_{1}, \ldots, X_{n-1}$ which violates inequality $n-1$. Recall that if we take a second hyperplane of the form $V_{2} \cup V_{j}$, for an arbitrary $j \in\{3, \ldots, n\}$ and relax it, this yields a representable matroid $\operatorname{Kin}(n)_{j}^{\bar{j}}$ by Lemma 3.8. As relaxing a circuit-hyperplane causes that subset to become independent, the rank of $V_{2} \cup V_{j}$ increases by one. The rank of all other subsets are unchanged. Suppose $V_{2} \cup V_{j}$ was not a term in inequality $n-1$ as applied to $X_{1}, \ldots, X_{n-1}$. Relaxing $V_{2} \cup V_{j}$ would then have no effect on the value of the inequality, giving that $X_{1}, \ldots, X_{n-1}$ is a bad family in $\operatorname{Kin}(n)=$. This contradicts the matroid being representable. Suppose that $V_{2} \cup V_{j}$ is a term on the left-hand side of inequality $n-1$. Then, when we relax $V_{2} \cup V_{j}$, the left-hand side of the inequality increases by one while the right-hand side remains the same. As the inequality previously did not hold, that is, the left-hand side was in fact greater than the right-hand side, it still cannot hold. Thus $V_{2} \cup V_{j}$ must be a term on the right-hand side of inequality $n$, for all $j$. There are $n-2$ choices of $j$, hence $n-2$ terms on the right-hand side of inequality $n-1$ must be these circuit-hyperplanes, $V_{2} \cup V_{j}$.

Next, note that if we tighten $V_{1} \cup V_{2}$, the resulting matroid is representable, by [5. Lemma 4.6]. This decreases the rank of $V_{1} \cup V_{2}$ by one and leaves the ranks of all other subsets unchanged. As with $V_{2} \cup V_{j}$, the set $V_{1} \cup V_{2}$ must be a term of inequality $n-1$ in order for the inequality to be able to reflect the change in representability. If $V_{1} \cup V_{2}$ was a term on the right-hand side, after tightening $V_{1} \cup$ $V_{2}$ the right-hand side would decrease by one and the left-hand side would remain the same. As prior to tightening the left-hand side was greater than the right-hand 
side, this fact is still true, meaning the inequality does not hold, contradicting the matroid being representable. A term on the left-hand side of inequality $n-1$ must be equal to $V_{1} \cup V_{2}$.

Now consider a graph $G$ on vertices $V=\left\{X_{1}, \ldots, X_{n-1}\right\}$ with adjacency relation $a$ such that

$$
a\left(X_{i}\right)=\left\{X_{j}\left|X_{i} \cup X_{j}\right| \text { is a term on the right-hand side of inequality } n-1\right\}
$$

Take the subgraph $G^{\prime}$ induced by the edges of $G$ corresponding to the circuithyperplanes $V_{2} \cup V_{3}, \ldots, V_{2} \cup V_{n}$. Suppose $G^{\prime}$ has a path of length three. Call this path $X_{a}, e, X_{b}, f, X_{c}, g, X_{d}$ and let the edges $e, f, g$ refer to the circuit-hyperplanes $V_{2} \cup V_{i}, V_{2} \cup V_{j}, V_{2} \cup V_{k}$ respectively.

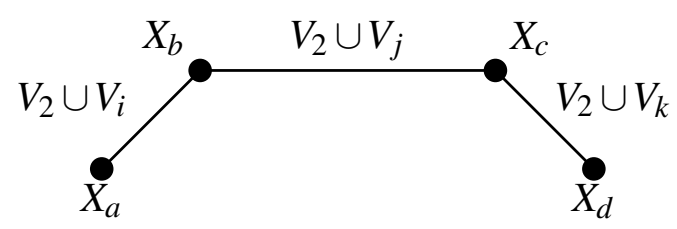

Consider what elements must lie where; $X_{b} \cup X_{c}$ must be equal to $V_{2} \cup V_{j}$. The set $X_{b}$ cannot contain any elements of $V_{j}$ as $X_{a} \cup X_{b}=V_{2} \cup V_{i}$, and $V_{i}$ and $V_{j}$ are disjoint. However, $X_{c}$ cannot contain any of the elements of $V_{j}$ either, since $X_{c} \cup$ $X_{d}=V_{2} \cup V_{k}$ does not. This structure can thus not exist, that is, $G^{\prime}$ can have no paths of length three. The same reasoning shows $G^{\prime}$ cannot have a cycle of length three. We will now show that $G^{\prime}$ does in fact contain a path or cycle of length three. As $V_{1} \cup V_{2}$ must be a term on the left-hand side of inequality $n-1$, one of $X_{1}, \ldots, X_{n-1}$ must contain elements from $V_{1}$. This subset cannot be incident with any edge representing a circuit-hyperplane $V_{2} \cup V_{i}$, so $G^{\prime}$ has at most $n-2$ vertices. $G^{\prime}$ must have $n-2$ edges. As trees must have an edge set of size one less than the number of vertices, this shows $G^{\prime}$ is not a tree, and thus contains a path or cycle of length three. Hence $\operatorname{Kin}(n)^{-}$cannot have a bad family for inequality $n-1$. 


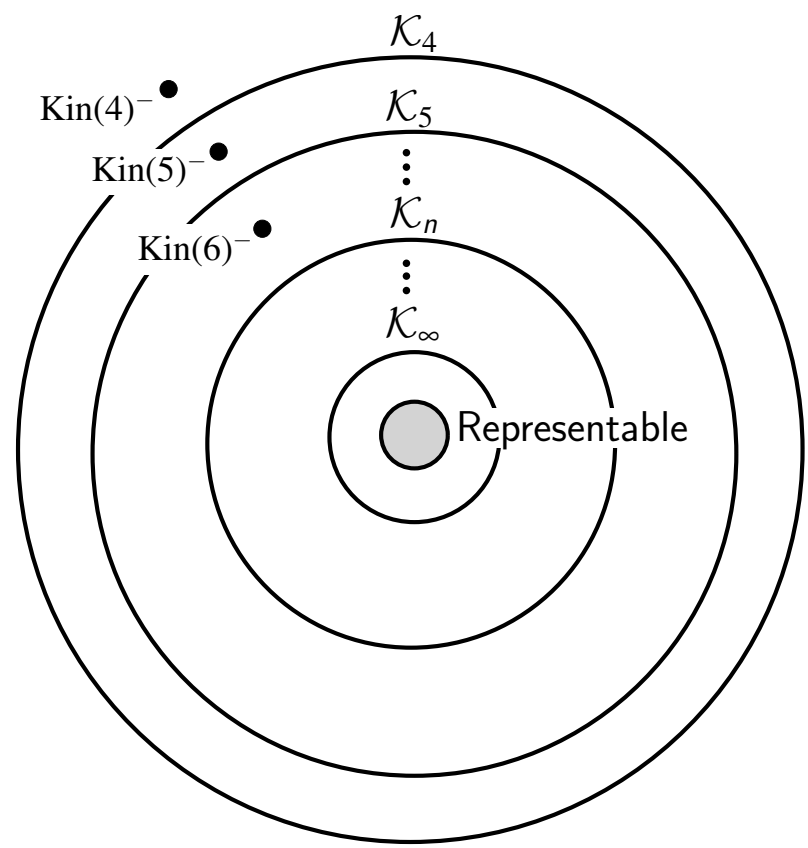

Figure 4.2: Kinser classes (2)

Next we show that the first Kinser class, $\mathcal{K}_{4}$, is dual-closed.

Lemma 4.4. Let $M$ be an excluded minor for the class $\mathcal{K}_{4}$. Let $X_{1}, \ldots, X_{4}$ be a bad family in $M$ for inequality 4 . Then $X_{1}, \ldots, X_{4}$ is a partition of $E(M)$.

Proof. Suppose there is an element of $E(M)$ which is not contained in some $X_{i}$. We could then delete this element and $X_{1}, \ldots, X_{n}$ would still form a bad family, contradicting $M$ being minor-minimal with respect to not being in $\mathcal{K}_{4}$. Thus $X_{1}, \ldots, X_{n}$ must cover the entire ground set.

Now assume the sets in the bad family are not disjoint, so there exists an $x$ which is in $X_{i} \cap X_{j}$ for some $i, j$. Contract $x$, and for each set $X_{k}$, let $X_{k}^{\prime}=X_{k}-\{x\}$.

Let $L$ be some union of the $X_{i}$ 's, so that $r(L)$ appears somewhere in the inequality. Say that $L$ is stable if $r_{M / x}(L-\{x\})=r_{M}(L)$. If none of the five terms on the right-hand side of the inequality are stable, then the sum on the right-hand side of the inequality decreases by exactly five when we contract $x$ and remove $x$ from each term. Since the left-hand side can decrease by at most five, this means that $X_{1} / x, \ldots, X_{n} / x$ is a bad family in $M / x$, which is impossible as $M$ is an excluded minor for $\mathcal{K}_{4}$, and so $M / x \in \mathcal{K}_{4}$. Therefore there is a stable set on the right-hand side.

As $x \in X_{i} \cap X_{j}$, the rank of any term that includes $X_{i}$ or $X_{j}$ decreases when we contract $x$ and remove it from the term, and so these terms cannot be stable. There 
is at most one term on the right-hand side of the inequality that does not involve $X_{i}$ or $X_{j}$, so the right-hand side has exactly one stable term. Now this stable term is equal to $X_{m} \cup X_{n}$, where either $m$ or $n$ is equal to 3 or 4 . Note $x \notin \operatorname{cl}\left(X_{m}\right)$ and $x \notin \operatorname{cl}\left(X_{n}\right)$, or else $X_{m} \cup X_{n}$ would not be stable. Therefore either $X_{3}$ or $X_{4}$ is stable, so there is a stable term on the left-hand side of the inequality also. This means that $X_{1} / x, \ldots, X_{n} / x$ is a bad family in $M / x$, and we get another contradiction.

Lemma 4.5. $\mathcal{K}_{4}=\mathcal{K}_{4}^{*}$

Proof. Assume for a contradiction that $M \in \mathcal{K}_{4}$ and $M^{*} \notin \mathcal{K}_{4}$. Note that $M^{*}$ contains a minor-minimal matroid not in $\mathcal{K}_{4}$. Let $N$ be a minor of $M$ such that $N^{*}$ is an excluded minor for $\mathcal{K}_{4}$. Let $X_{1}, \ldots, X_{4}$ be a bad family of $N^{*}$. By the previous lemma, we have that $X_{1}, \ldots, X_{4}$ partitions $E\left(N^{*}\right)$. By assumption we have that

$$
\begin{aligned}
& r^{*}\left(X_{3}\right)+r^{*}\left(X_{4}\right)+r^{*}\left(X_{1} \cup X_{2}\right)+r^{*}\left(X_{1} \cup X_{3} \cup X_{4}\right)+r^{*}\left(X_{2} \cup X_{3} \cup X_{4}\right) \\
& \leq r^{*}\left(X_{1} \cup X_{3}\right)+r^{*}\left(X_{1} \cup X_{4}\right)+r^{*}\left(X_{2} \cup X_{3}\right)+r^{*}\left(X_{2} \cup X_{4}\right)+r^{*}\left(X_{3} \cup X_{4}\right)
\end{aligned}
$$

Recall $r^{*}(X)=|X|+r(\bar{X})-r(N)$ where $\bar{X}=E(N)-X$. Use this identity on every term in the inequality. Now we see that 4.5.1) is true if and only if 4.5.2) is true.

$$
\begin{aligned}
\left|X_{3}\right| & +\left|X_{4}\right|+r\left(\overline{X_{3}}\right)+r\left(\overline{X_{4}}\right)+\left|X_{1} \cup X_{2}\right|+r\left(\overline{X_{1} \cup X_{2}}\right) \\
& +\left|X_{1} \cup X_{3} \cup X_{4}\right|+r\left(\overline{X_{1} \cup X_{3} \cup X_{4}}\right) \\
& +\left|X_{2} \cup X_{3} \cup X_{4}\right|+r\left(\overline{X_{2} \cup X_{3} \cup X_{4}}\right)-5 r(N) \\
\not \leq \mid & X_{1} \cup X_{3}\left|+r\left(\overline{X_{1} \cup X_{3}}\right)+\right| X_{1} \cup X_{4} \mid+r\left(\overline{X_{1} \cup X_{4}}\right) \\
& +\left|X_{2} \cup X_{3}\right|+r\left(\overline{X_{2} \cup X_{3}}\right)+\left|X_{2} \cup X_{4}\right| \\
& +r\left(\overline{X_{2} \cup X_{4}}\right)+\left|X_{3} \cup X_{4}\right|+r\left(\overline{X_{2} \cup X_{4}}\right)-5 r(N)
\end{aligned}
$$

Using the identities $\left|X_{i} \cup X_{j}\right|=\left|X_{i}\right|+\left|X_{j}\right|-\left|X_{1} \cap X_{j}\right|$ and $\left|X_{i} \cup X_{j} \cup X_{k}\right|=\left|X_{i}\right|+$ $\left|X_{j}\right|+\left|X_{k}\right|-\left|X_{i} \cap X_{j}\right|-\left|X_{i} \cap X_{k}\right|-\left|X_{j} \cap X_{k}\right|+\left|X_{i} \cap X_{j} \cap X_{k}\right|$, we can simplify this.

Fully apply these identities to each cardinality term. We now have that 4.5 .2 is 
true if and only if 4.5 .3 is true.

$$
\begin{aligned}
\left|X_{3}\right|+ & \left|X_{4}\right|+r\left(\overline{X_{3}}\right)+r\left(\overline{X_{4}}\right) \\
& +\left|X_{1}\right|+\left|X_{2}\right|-\left|X_{1} \cap X_{2}\right|+r\left(\overline{X_{1} \cup X_{2}}\right) \\
& +\left|X_{1}\right|+\left|X_{3}\right|+\left|X_{4}\right|-\left|X_{1} \cap X_{3}\right|-\left|X_{1} \cap X_{4}\right| \\
& \quad-\left|X_{3} \cap X_{4}\right|+\left|X_{1} \cap X_{3} \cap X_{4}\right|+r\left(\overline{X_{1} \cup X_{3} \cup X_{4}}\right) \\
& +\left|X_{2}\right|+\left|X_{3}\right|+\left|X_{4}\right|-\left|X_{2} \cap X_{3}\right|-\left|X_{2} \cap X_{4}\right|-\left|X_{3} \cap X_{4}\right| \\
& \quad+\left|X_{2} \cap X_{3} \cap X_{4}\right|+r\left(\overline{X_{2} \cup X_{3} \cup X_{4}}\right) \\
\not \leq\left|X_{1}\right|+\left|X_{3}\right|-\left|X_{1} \cap X_{3}\right|+r\left(\overline{X_{1} \cup X_{3}}\right) & \\
& +\left|X_{1}\right|+\left|X_{4}\right|-\left|X_{1} \cap X_{4}\right|+r\left(\overline{X_{1} \cup X_{4}}\right) \\
& +\left|X_{2}\right|+\left|X_{3}\right|-\left|X_{2} \cap X_{3}\right|+r\left(\overline{X_{2} \cup X_{3}}\right) \\
& +\left|X_{2}\right|+\left|X_{4}\right|-\left|X_{2} \cap X_{4}\right|+r\left(\overline{X_{2} \cup X_{4}}\right) \\
& +\left|X_{3}\right|+\left|X_{4}\right|-\left|X_{3} \cap X_{4}\right|+r\left(\overline{X_{2} \cup X_{4}}\right)
\end{aligned}
$$

As $X_{1}, \ldots, X_{4}$ is a partition of $E(M)$, any term $X_{i} \cap X_{j}$ is empty. Cancelling out these terms, and all common terms, yields

$$
\begin{aligned}
& r\left(\overline{X_{3}}\right)+r\left(\overline{X_{4}}\right)+r\left(\overline{X_{1} \cup X_{2}}\right)+r\left(\overline{X_{1} \cup X_{3} \cup X_{4}}\right)+r\left(\overline{X_{2} \cup X_{3} \cup X_{4}}\right) \\
\not \leq & r\left(\overline{X_{1} \cup X_{3}}\right)+r\left(\overline{X_{1} \cup X_{4}}\right)+r\left(\overline{X_{2} \cup X_{3}}\right)+r\left(\overline{X_{2} \cup X_{4}}\right)+r\left(\overline{X_{3} \cup X_{4}}\right)
\end{aligned}
$$

Now let $Y_{1}=\overline{X_{1} \cup X_{2} \cup X_{3}}, \quad Y_{2}=\overline{X_{1} \cup X_{2} \cup X_{4}}, \quad Y_{3}=\overline{X_{1} \cup X_{3} \cup X_{4}}$, and $Y_{4}=$ $\overline{X_{2} \cup X_{3} \cup X_{4}}$.

We have the following equalities

$$
\begin{aligned}
Y_{1} \cup Y_{3} \cup Y_{4} & =\overline{X_{1} \cup X_{2} \cup X_{3}} \cup \overline{X_{1} \cup X_{3} \cup X_{4}} \cup \overline{X_{2} \cup X_{3} \cup X_{4}} \\
& =X_{4} \cup X_{2} \cup X_{1} \\
& =\overline{X_{3}} \\
Y_{2} \cup Y_{3} \cup Y_{4} & =\overline{X_{1} \cup X_{2} \cup X_{4}} \cup \overline{X_{1} \cup X_{3} \cup X_{4}} \cup \overline{X_{2} \cup X_{3} \cup X_{4}} \\
& =X_{3} \cup X_{2} \cup X_{1} \\
& =\overline{X_{4}}
\end{aligned}
$$




$$
\begin{aligned}
& Y_{1} \cup Y_{2}=\overline{X_{1} \cup X_{2} \cup X_{3}} \cup \overline{X_{1} \cup X_{2} \cup X_{4}} \\
& =X_{4} \cup X_{3} \\
& =\overline{X_{1} \cup X_{2}} \\
& Y_{1} \cup Y_{3}=\overline{X_{1} \cup X_{2} \cup X_{3}} \cup \overline{X_{1} \cup X_{3} \cup X_{4}} \\
& =X_{4} \cup X_{2} \\
& =\overline{X_{1} \cup X_{3}} \\
& Y_{2} \cup Y_{3}=\overline{X_{1} \cup X_{2} \cup X_{4}} \cup \overline{X_{1} \cup X_{3} \cup X_{4}} \\
& =X_{3} \cup X_{2} \\
& =\overline{X_{1} \cup X_{4}} \\
& Y_{1} \cup Y_{4}=\overline{X_{1} \cup X_{2} \cup X_{3}} \cup \overline{X_{2} \cup X_{3} \cup X_{4}} \\
& =X_{4} \cup X_{1} \\
& =\overline{X_{2} \cup X_{3}} \\
& Y_{2} \cup Y_{4}=\overline{X_{1} \cup X_{2} \cup X_{4}} \cup \overline{X_{2} \cup X_{3} \cup X_{4}} \\
& =X_{3} \cup X_{1} \\
& =\overline{X_{2} \cup X_{4}} \\
& Y_{3} \cup Y_{4}=\overline{X_{1} \cup X_{3} \cup X_{4}} \cup \overline{X_{2} \cup X_{3} \cup X_{4}} \\
& =X_{2} \cup X_{1} \\
& =\overline{X_{3} \cup X_{4}}
\end{aligned}
$$

Inequality (4.5.4) thus becomes

$$
\begin{aligned}
& r\left(Y_{1} \cup Y_{3} \cup Y_{4}\right)+r\left(Y_{2} \cup Y_{3} \cup Y_{4}\right)+r\left(Y_{1} \cup Y_{2}\right)+r\left(Y_{3}\right)+r\left(Y_{4}\right) \\
& \not \leq r\left(Y_{1} \cup Y_{3}\right)+r\left(Y_{2} \cup Y_{3}\right)+r\left(Y_{1} \cup Y_{4}\right)+r\left(Y_{2} \cup Y_{4}\right)+r\left(Y_{3} \cup Y_{4}\right)
\end{aligned}
$$

These rank terms are exactly those of inequality 4 , which holds for $Y_{1}, \ldots, Y_{4}$ as 
$N \in \mathcal{K}_{4}$. Thus $N^{*}$ also satisfies inequality 4 , for all choices of $X_{1}, \ldots, X_{4}$.

We conjecture that this is the only Kinser class which is dual-closed. We provide a proof that the second Kinser class is indeed not dual-closed. There appears to be no simple way to verify that a matroid satisfies a particular Kinser inequality, which leads to the difficulty involved in the next chapter. In a later chapter, we will provide a result on the complexity of verifying that a matroid satisfies Kinser inequality $n$.

Theorem 4.6. $\mathcal{K}_{5} \neq \mathcal{K}_{5}^{*}$

The proof of this theorem forms the content of the next chapter. There are now two possibilities of how the Kinser classes sit in the hierarchy, as shown in the following diagrams.

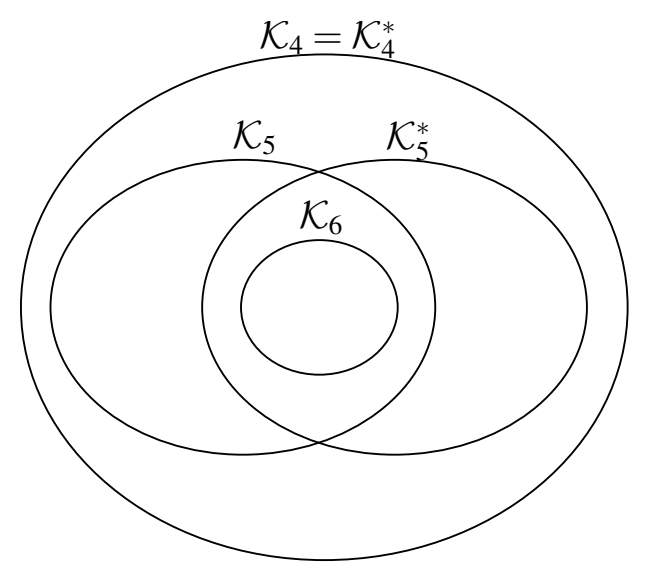

Figure 4.3: Kinser classes (3a)

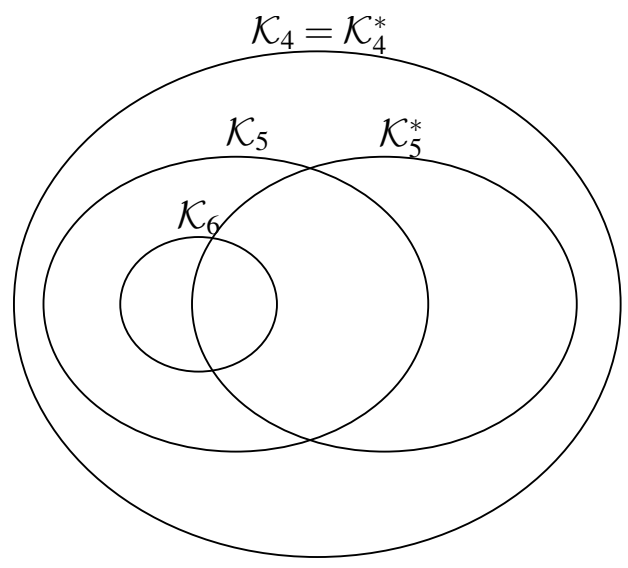

Figure 4.4: Kinser classes (3b) 


\section{Chapter 5}

\section{$\mathcal{K}_{5} \neq \mathcal{K}_{5}^{*}$}

Theorem 5.1. $\operatorname{Kin}(5)^{-} \in \mathcal{K}_{5}^{*}$.

Proof. Recall that $\operatorname{Kin}(5)^{-}$is the matroid obtained from the rank-5 Kinser matroid by relaxing the circuit-hyperplane $V_{1} \cup V_{2}$. Assume $\left(\operatorname{Kin}(5)^{-}\right)^{*}$ has at least one bad family $X_{1}, \ldots, X_{5}$ violating Kinser inequality 5 , where each set $X_{i}$ is a flat of $\left(\operatorname{Kin}(5)^{-}\right)^{*}$.

Note that the complement of $V_{1} \cup V_{2}$ is a relaxed circuit-hyperplane in $\left(\operatorname{Kin}(5)^{-}\right)^{*}$, and tightening it produces $\operatorname{Kin}(5)^{*}$, which is representable. This operation affects the rank function by decreasing the rank of exactly one set: $V_{3} \cup V_{4} \cup V_{5}$. Suppose $V_{3} \cup V_{4} \cup V_{5}$ is not a term in inequality 5 as applied to $X_{1}, \ldots, X_{5}$. Then evaluating the inequality after relaxing the circuit-hyperplane would give the same value as before relaxing, contradicting the change in representability. As the rank of $V_{3} \cup$ $V_{4} \cup V_{5}$ decreases by one when we tighten it, the only way for the inequality to hold only after the tightening is for the left-hand side of the inequality to decrease and the right-hand side to remain the same. So $V_{3} \cup V_{4} \cup V_{5}$ must be a term on the left-hand side of the inequality.

Next note that $V_{2} \cup V_{3}, V_{2} \cup V_{4}$, and $V_{2} \cup V_{5}$ are circuit-hyperplanes in $\operatorname{Kin}(5)^{-}$, so $V_{1} \cup V_{4} \cup V_{5}, V_{1} \cup V_{3} \cup V_{5}, V_{1} \cup V_{3} \cup V_{4}$ are circuit-hyperplanes of $\left(\operatorname{Kin}(5)^{-}\right)^{*}$. Relaxing any one of these would yield a representable matroid, and so these subsets must be terms in the inequality. The rank of each of these subsets increases by one when relaxed, so for the inequality to hold the right-hand side must increase, meaning these three sets must be terms on the right-hand side of inequality 5 .

Let $e \in V_{2}$. If $e \notin X_{1}, \ldots, X_{5}$, then $\left(\operatorname{Kin}(5)^{-}\right)^{*} \backslash e=\left(\operatorname{Kin}(5)^{-} / e\right)^{*}$ has a bad family. 
This means that $\operatorname{Kin}(5)^{-} / e$ is not representable. We will show that the elements of $V_{1}$ are freely placed in $\operatorname{Kin}(5)^{-} / e$. Pick $z \in V_{1}$. Let $Z$ be a non-spanning subset in $\operatorname{Kin}(5)^{-} / e$ such that $z \in \mathrm{cl}_{\operatorname{Kin}(5)^{-} / e}(Z)-Z$. This implies by Lemma $2.5 z \in \mathrm{cl}_{\mathrm{Kin}(5)^{-}}(Z \cup\{e\})$. Note that the closure of a set in $\operatorname{Kin}(5)$ only differs from that in $\operatorname{Kin}(5)^{-}$in that it may contain additional elements in $\operatorname{Kin}(5)$. Thus $z \in \mathrm{cl}_{\operatorname{Kin}(5)}(Z \cup\{e\})$.

Recall that $M_{6}$ is the transversal matroid whose truncation is defined to be $\operatorname{Kin}(5)$. As $Z \cup\{e\}$ does not span $\operatorname{Kin}(5)$, truncation does not affect the rank of this subset, and so $z \in \operatorname{cl}_{M_{6}}(Z \cup\{e\})$. Now consider the neighbours of $Z \cup\{e\}$ in the transversal system $\mathcal{A}$. The neighbours of $z$ must be contained in this set, as otherwise the rank of $Z \cup\{e, z\}$ would be greater than the rank of $Z \cup\{e\}$, contradicting $z \in \operatorname{cl}(Z \cup\{e\})$. Recall that in $M_{r+1}, V_{1}$ is incident with $A_{4}, A_{5}$ and $A$, while $A_{2}$ is incident with $A^{\prime}$. As $z \in V_{1}$ and $e \in V_{2}$, we have that $Z \cup\{e\}$ is neighbours with $A_{4}, A_{5}, A$, and $A^{\prime}$.

$\operatorname{Recall} Z$ is non-spanning in $\operatorname{Kin}(5)^{-} / e$. As $\operatorname{Kin}(5)^{-} / e$ has $\operatorname{rank} 4, r_{\operatorname{Kin}(5)^{-} / e}(Z) \leq$ 3 , and $r_{\operatorname{Kin}(5)^{-}}(Z \cup\{e\}) \leq 4$. This rank cannot change after tightening $V_{1} \cup V_{2}$, so $r_{\operatorname{Kin}(5)}(Z \cup\{e\}) \leq 4$. Hence $Z \cup\{e\}$ is non-spanning in $\operatorname{Kin}(5)$, so $r_{M_{6}}(Z \cup$ $\{e\} \leq 4$. This means that $Z \cup\{e\}$ has exactly $A_{0}, A_{1}, A_{3}$, and $A_{4}$ as neighbours. Thus $Z \cup\{e\} \subseteq V_{1} \cup\{e, f\}$. This implies that $Z \cup\{e, z\}$ is independent in $\operatorname{Kin}(5)^{-}$, and so $Z \cup\{z\}$ is independent in $\operatorname{Kin}(5)^{-} / e$. This is a contradiction, as $z \in \mathrm{cl}_{\operatorname{Kin}(5)^{-} / e}(Z)-Z$. Thus $z$ is free in $\operatorname{Kin}(5)^{-} / e$ and $\operatorname{Kin}(5)^{-} / e$ is a freeextension of $\operatorname{Kin}(5)^{-} / e \backslash z=\operatorname{Kin}(5) / e \backslash z$ which is $\mathbb{K}$-representable. This contra$\operatorname{dicts}\left(\operatorname{Kin}(5)^{-}\right)^{*} \backslash e=\left(\operatorname{Kin}(5)^{-} / e\right)^{*}$ having a bad family, and so all elements of $V_{2}$ must be contained in some set $X_{i}$.

Suppose the elements of $V_{2}$ are contained in different sets in the bad family. That is, suppose $e \in X_{i}$ and $f \in X_{j}$ for some $i, j$. The three circuit-hyperplanes $V_{1} \cup$ $V_{3} \cup V_{4}, V_{1} \cup V_{4} \cup V_{5}, V_{1} \cup V_{3} \cup V_{5}$ and the relaxed circuit-hyperplane $V_{3} \cup V_{4} \cup V_{5}$ do not include elements of $V_{2}$, and thus cannot be equal to terms in the inequality which use $X_{i}$ or $X_{j}$. Removing these terms from the inequality leaves at most three possible terms for these circuit-hyperplanes, regardless of the values of $i$ and $j$. Thus both elements of $V_{2}, e$ and $f$, must be contained in the same set $X_{i}$ of the bad family.

Considering each possible location for the elements of $V_{2}$ gives us five cases $V_{2} \cap X_{1} \neq \varnothing, V_{2} \cap X_{2} \neq \varnothing, V_{2} \cap X_{3} \neq \varnothing, V_{2} \cap X_{4} \neq \varnothing$, and $V_{2} \cap X_{5} \neq \varnothing$. Take 
the case $X_{5} \cap V_{2} \neq \varnothing$. Swapping $X_{3}$ and $X_{5}$ gives the inequality, and this case is thus identical to the case where $V_{2} \cap X_{3} \neq \varnothing$. We thus reduce to the four cases examined below.

Case 1. $X_{3} \cap V_{2} \neq \varnothing$

Consider which term on the left-hand side of the inequality must be $V_{3} \cup V_{4} \cup V_{5}$. As this term does not contain any elements of $V_{2}$ and these elements are contained in $X_{3}$, the term cannot involve $X_{3}$. In the following diagram, the ovals represent the sets $X_{1}, \ldots, X_{5}$. An edge between two sets represents the union of those two sets. The edges shown below are on the right-hand side of the inequality, and thus possible locations for the necessary circuit-hyperplanes $V_{1} \cup V_{3} \cup V_{5}, V_{1} \cup V_{4} \cup V_{5}$, $V_{1} \cup V_{3} \cup V_{4}$. Note that $X_{1} \cup X_{2}$, indicated by the dashed line, appears on the lefthand side of the inequality. The edges coming from $X_{3}$ have been left off as, since the elements of $V_{2}$ are contained in $X_{3}$, none of these edges could represent the three circuit-hyperplanes.
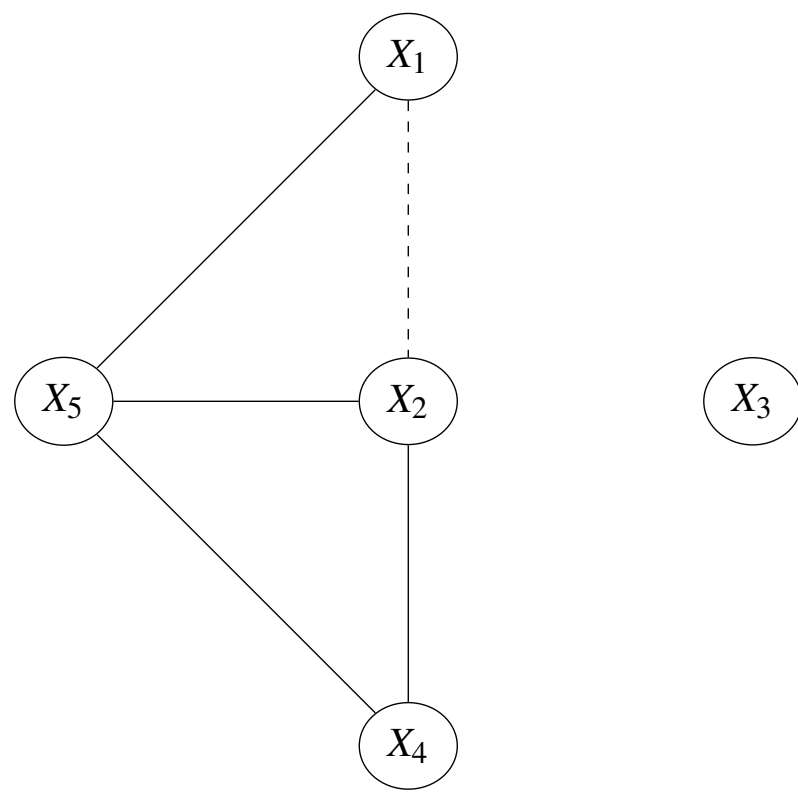

If $V_{3} \cup V_{4} \cup V_{5}$ is equal to $X_{4}$ or $X_{5}$ then any term involving these sets cannot be one of the circuit-hyperplanes $V_{1} \cup V_{3} \cup V_{5}, V_{1} \cup V_{4} \cup V_{5}, V_{1} \cup V_{3} \cup V_{4}$ as these must appear on the right-hand side of the inequality. This will not leave us with enough terms on the right-hand side which could be these three circuit-hyperplanes - if $V_{3} \cup V_{4} \cup V_{5}=X_{4}$, then the terms available for the circuit-hyperplanes are $X_{1} \cup X_{5}$ and $X_{2} \cup X_{5}$, while if $V_{3} \cup V_{4} \cup V_{5}=X_{5}$, there are again only two terms available, 
$X_{1} \cup X_{2}$ and $X_{2} \cup X_{4}$. If $V_{3} \cup V_{4} \cup V_{5}=X_{2} \cup X_{4} \cup X_{5}$, then elements of $V_{1}$ can only be in $X_{1}$. Note that all of the three circuit-hyperplanes which must appear on the right-hand side include $V_{1}$. However, as $X_{3} \cap V_{2} \neq \varnothing$, the only free term using $X_{1}$ is $X_{1} \cup X_{5}$, so we are unable to have the three needed circuit-hyperplanes on the right-hand side. The only remaining possibility is that $V_{3} \cup V_{4} \cup V_{5}=X_{1} \cup X_{2}$.

We now have four possibilities of where the circuit-hyperplanes on the right-hand side of the inequality lie, and we will consider various subcases. Let $\{i, j, k\}=$ $\{3,4,5\}$. The following diagrams show these subcases, as indicated by the bold lines.

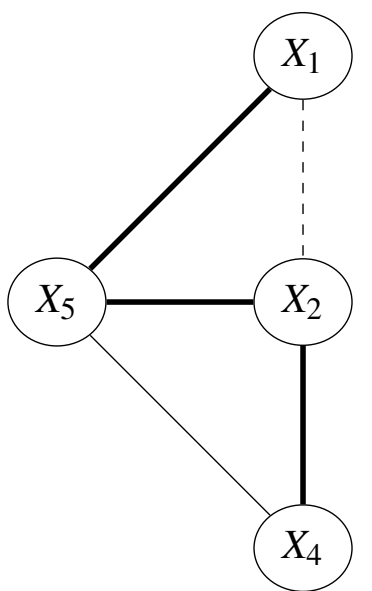

Subcase 1.1

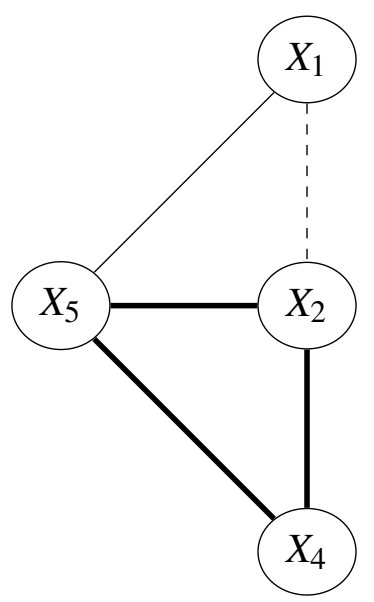

Subcase 1.3

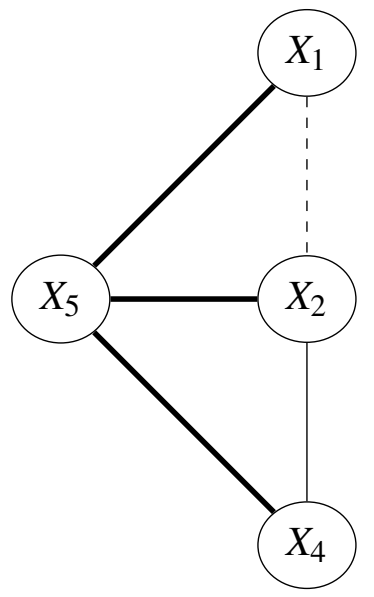

Subcase 1.2

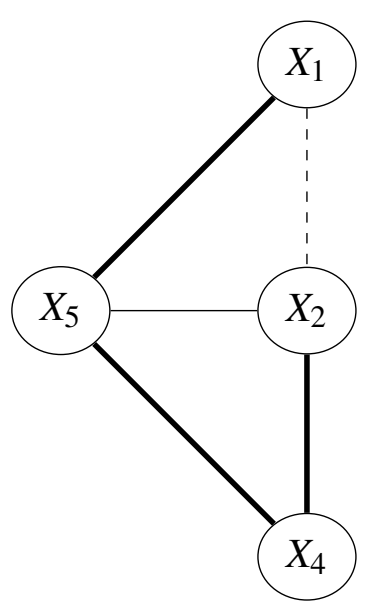

Subcase 1.4
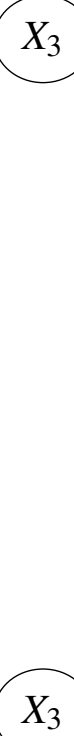
In each subcase examined below, we are considering the circuit-hyperplanes to lie as indicated by the diagram at the start of each subcase. In all of these, there are multiple options for which elements are in what sets, as described below.

\section{Subcase 1.1.}

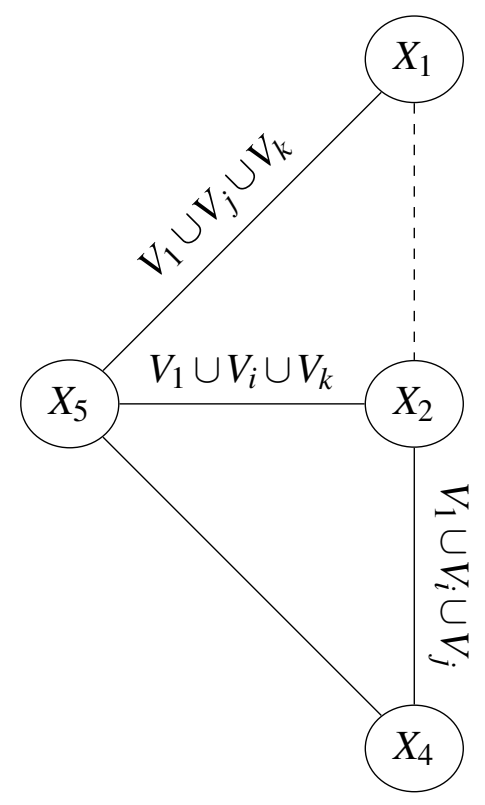

Here, we have assumed that $V_{2} \subseteq X_{3}$. As $X_{3}$ may contain other elements of the ground set of $M$, any terms in the inequality involving $X_{3}$ will not be immediately evaluated. Recall that $X_{1} \cup X_{2}=V_{3} \cup V_{4} \cup V_{5}$. This means that $V_{1}$ can only be a subset of $X_{4}$ or $X_{5}$. In order for the three circuit-hyperplanes to lie as indicated, both $X_{4}$ and $X_{5}$ must contain $V_{1}$. Also, $X_{2}$ must be equal to $V_{i}$, as this forms the common values of the two circuit-hyperplanes involving $X_{2}$, excluding $V_{1}$. Finally, $X_{5}$ must be equal to $V_{1} \cup V_{k}$, while $X_{1}$ must contain $V_{j} \cup V_{k}$. As we assumed the sets $X_{i}$ to be flats, $X_{1}$ could also include one or two elements of $V_{i}$. It cannot contain all three elements, as $V_{i} \cup V_{j} \cup V_{k}$ is a relaxed circuit-hyperplane, and thus has closure equal to the entire ground set. This gives the following three subcases, which differ only in elements of $X_{1}$. In each subcase, we will begin by evaluating terms of the left-hand and right-hand sides of inequality 5 , then show that the left-hand side must be lower in value, meaning that the inequality holds.

Subcase 1.1a: $X_{1}=V_{j} \cup V_{k}$

$X_{2}=V_{i}$

$X_{3}=V_{2} \cup Z$ where $Z \subseteq E(M)$ 


$$
\begin{aligned}
& X_{4}=V_{1} \cup V_{j} \\
& X_{5}=V_{1} \cup V_{k}
\end{aligned}
$$

Note that $\overline{X_{1} \cup X_{2}}=V_{1} \cup V_{2}$ and that $\overline{X_{2} \cup X_{4} \cup X_{5}}=\overline{V_{1} \cup V_{i} \cup V_{j} \cup V_{k}}=V_{2}$.

We will use $M$ to refer to $\operatorname{Kin}(5)^{-}$.

$$
\begin{aligned}
\text { LHS }= & r^{*}\left(X_{3}\right)+r^{*}\left(X_{4}\right)+r^{*}\left(X_{5}\right)+r^{*}\left(X_{1} \cup X_{2}\right)+r^{*}\left(X_{1} \cup X_{3} \cup X_{5}\right) \\
& +r^{*}\left(X_{2} \cup X_{3} \cup X_{4}\right)+r^{*}\left(X_{2} \cup X_{4} \cup X_{5}\right) \\
=r^{*} & \left(X_{3}\right)+r^{*}\left(X_{1} \cup X_{3} \cup X_{5}\right)+r^{*}\left(X_{2} \cup X_{3} \cup X_{4}\right) \\
& +\left|X_{4}\right|+\left|X_{5}\right|+\left|X_{1} \cup X_{2}\right|+\left|X_{2} \cup X_{4} \cup X_{5}\right| \\
& +r\left(\overline{X_{4}}\right)+r\left(\overline{X_{5}}\right)+r\left(\overline{X_{1} \cup X_{2}}\right)+r\left(\overline{X_{2} \cup X_{4} \cup X_{5}}\right)-4 r(M) \\
=r^{*}\left(X_{3}\right)+r^{*}\left(X_{1} \cup X_{3} \cup X_{5}\right)+r^{*}\left(X_{2} \cup X_{3} \cup X_{4}\right)-4 r(M) & +6+6+9+12+5+5+5+2 \\
& +6+X^{*}\left(X_{3}\right)+r^{*}\left(X_{1} \cup X_{3} \cup X_{5}\right)+r^{*}\left(X_{2} \cup X_{3} \cup X_{4}\right)-4 r(M)+50 \\
= & \left.r^{*}\right) \\
R H S= & \left.r^{*}\left(X_{1} \cup X_{3}\right)+r^{*}\left(X_{1} \cup X_{5}\right)+r^{*}\left(X_{2} \cup X_{3}\right)+X_{2} \cup X_{4}\right) \\
& +r^{*}\left(X_{2} \cup X_{5}\right)+r^{*}\left(X_{3} \cup X_{4}\right)+r^{*}\left(X_{4} \cup X_{5}\right) \\
= & r^{*}\left(X_{1} \cup X_{3}\right)+r^{*}\left(X_{2} \cup X_{3}\right)+r^{*}\left(X_{3} \cup X_{4}\right)+\left|X_{1} \cup X_{5}\right| \\
& +\left|X_{2} \cup X_{4}\right|+\left|X_{2} \cup X_{5}\right|+\left|X_{4} \cup X_{5}\right|+r\left(\overline{X_{1} \cup X_{5}}\right) \\
& +r\left(\overline{X_{2} \cup X_{4}}\right)+r\left(\overline{X_{2} \cup X_{5}}\right)+r\left(\overline{X_{4} \cup X_{5}}\right)-4 r(M) \\
\geq & r^{*}\left(X_{1} \cup X_{3}\right)+r^{*}\left(X_{2} \cup X_{3}\right)+r^{*}\left(X_{3} \cup X_{4}\right)-4 r(M) \\
& +9+9+9+9+4+4+4+4 \\
= & r^{*}\left(X_{1} \cup X_{3}\right)+r^{*}\left(X_{2} \cup X_{3}\right)+r^{*}\left(X_{3} \cup X_{4}\right)-4 r(M)+52
\end{aligned}
$$

We must have that

$$
\begin{aligned}
& r^{*}\left(X_{3}\right)+r^{*}\left(X_{1} \cup X_{3} \cup X_{4}\right)+r^{*}\left(X_{2} \cup X_{3} \cup X_{5}\right) \\
> & r^{*}\left(X_{1} \cup X_{3}\right)+r^{*}\left(X_{2} \cup X_{3}\right)+r^{*}\left(X_{3} \cup X_{4}\right)+2
\end{aligned}
$$

in order for $X_{1}, \ldots, X_{5}$ to be a bad family.

Suppose $Z=\varnothing$, that is, $X_{3}=V_{2}$. We then have $2+9+9>8+5+8+2$ which is untrue. Suppose we increase the size of $Z$. If the cardinality of one of the unions in the above inequality does not change, then neither does the rank of 
the complement, and thus the corank is unchanged. Note that $X_{1} \cup X_{3} \cup X_{5}=$ $V_{1} \cup V_{2} \cup V_{j} \cup V_{k} \cup Z$. If the cardinality of this set is greater when $Z$ is non-empty, then $Z \subseteq V_{i}$. As $\overline{X_{1} \cup X_{3} \cup X_{5}}=V_{i}-Z$, this will cause the rank of $\overline{X_{1} \cup X_{3} \cup X_{5}}$ to decrease. The corank is thus unchanged from when $Z=\varnothing$. A similar argument shows that the corank of $X_{2} \cup X_{3} \cup X_{4}=V_{1} \cup V_{2} \cup V_{i} \cup V_{j} \cup Z$ must be unchanged as its complement $V_{k}-Z$ is independent. Also,

$$
\begin{aligned}
r^{*}\left(X_{3} \cup X_{4}\right)+2 & \geq r^{*}\left(X_{3}\right)+r^{*}\left(X_{4}\right)-r^{*}\left(X_{3} \cap X_{4}\right)+2 \\
& \geq r^{*}\left(X_{3}\right)+r^{*}\left(X_{4}\right)-r^{*}\left(Z \cap X_{4}\right)+2 \\
& \geq r^{*}\left(X_{3}\right)+2 \\
& \geq r^{*}\left(X_{3}\right)
\end{aligned}
$$

Thus increasing the size of $Z$ can only cause the left-hand side to decrease by more than the right-hand side, and so we cannot have a bad family for any choice of $Z$.

Subcase 1.1b: $X_{1}=V_{j} \cup V_{k} \cup\{a\}$ where $a \in V_{i}$

$$
\begin{aligned}
& X_{2}=V_{i} \\
& X_{3}=V_{2} \cup Z \text { where } Z \subseteq E(M) \\
& X_{4}=V_{1} \cup V_{j} \\
& X_{5}=V_{1} \cup V_{k}
\end{aligned}
$$

The only terms whose value changes from Subcase 1.1a is $\left|X_{1} \cup X_{5}\right|$ which increases by one. So we must have that

$$
\begin{aligned}
& r^{*}\left(X_{3}\right)+r^{*}\left(X_{1} \cup X_{3} \cup X_{4}\right)+r^{*}\left(X_{2} \cup X_{3} \cup X_{4}\right) \\
> & r^{*}\left(X_{1} \cup X_{3}\right)+r^{*}\left(X_{2} \cup X_{3}\right)+r^{*}\left(X_{3} \cup X_{4}\right)+3
\end{aligned}
$$

The same argument as before shows that we do not have a bad family, for any choice of $Z$.

Subcase 1c: $X_{1}=V_{j} \cup V_{k} \cup\{a, b\}$ where $a, b \in V_{i}$

$$
\begin{aligned}
& X_{2}=V_{i} \\
& X_{3}=V_{2} \cup Z \text { where } Z \subseteq E(M) \\
& X_{4}=V_{1} \cup V_{j} \\
& X_{5}=V_{1} \cup V_{k}
\end{aligned}
$$

Here, $\left|X_{1} \cup X_{5}\right|$ is one higher than in Subcase 1.1a, while $r\left(\overline{X_{1} \cup X_{5}}\right)$ is one lower, and the inequality is thus identical to subcase $1 \mathrm{~b}$. 


\section{Subcase 1.2.}
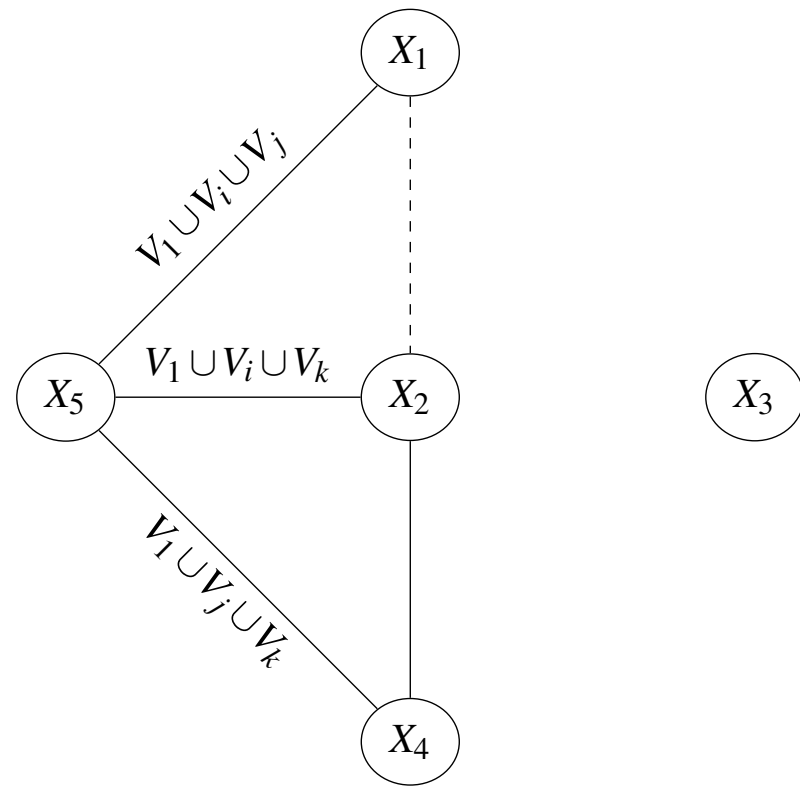

Again, we have by assumption that $V_{2} \subseteq X_{3} . X_{3}$ may contain other elements of the ground set of $M$, thus any terms in the inequality involving $X_{3}$ will not be immediately evaluated. $X_{5}$ must be equal to $V_{1}$, as these are the only common elements of the three circuit-hyperplanes. This forces $X_{1}$ to be equal to $V_{i} \cup V_{j}$, as $X_{1} \cup X_{2}=V_{3} \cup V_{4} \cup V_{5}$ and thus $X_{1}$ cannot contain elements of $V_{1}$. Likewise, $X_{2}$ must be equal to $V_{i} \cup V_{k}$. Also, $X_{4}$ must contain $V_{j} \cup V_{k}$. As we assumed that the sets $X_{i}$ are flats, $X_{4}$ could also include one or three elements of $V_{1}$. Note $X_{4}$ cannot contain exactly two elements of $V_{1}$, as $V_{1} \cup V_{j} \cup V_{k}$ is a circuit-hyperplane. This gives the following three subcases, which differ only in elements of $X_{4}$. As before, in each subcase we will begin by evaluating terms on the left-hand and right-hand sides of inequality 5 , then show that the left-hand side must be lower in value, meaning that the inequality holds.

Subcase 1.2a: $X_{1}=V_{i} \cup V_{j}$

$$
\begin{aligned}
& X_{2}=V_{i} \cup V_{k} \\
& X_{3}=V_{2} \cup Z \text { where } Z \subseteq E(M) \\
& X_{4}=V_{j} \cup V_{k} \\
& X_{5}=V_{1}
\end{aligned}
$$


Note that $\overline{X_{1} \cup X_{2}}=V_{1} \cup V_{2}$ and $\overline{X_{2} \cup X_{4} \cup X_{5}}=\overline{V_{1} \cup V_{i} \cup V_{j} \cup V_{k}}=V_{2}$.

$$
\begin{aligned}
\text { LHS }= & r^{*}\left(X_{3}\right)+r^{*}\left(X_{4}\right)+r^{*}\left(X_{5}\right)+r^{*}\left(X_{1} \cup X_{2}\right)+r^{*}\left(X_{1} \cup X_{3} \cup X_{5}\right) \\
& +r^{*}\left(X_{2} \cup X_{3} \cup X_{4}\right)+r^{*}\left(X_{2} \cup X_{4} \cup X_{5}\right) \\
= & r^{*}\left(X_{3}\right)+r^{*}\left(X_{1} \cup X_{3} \cup X_{5}\right)+r^{*}\left(X_{2} \cup X_{3} \cup X_{4}\right) \\
& +\left|X_{4}\right|+\left|X_{5}\right|+\left|X_{1} \cup X_{2}\right|+\left|X_{2} \cup X_{4} \cup X_{5}\right| \\
& +r\left(\overline{X_{4}}\right)+r\left(\overline{X_{5}}\right)+r\left(\overline{X_{1} \cup X_{2}}\right)+r\left(\overline{X_{2} \cup X_{4} \cup X_{5}}\right)-4 r(M) \\
=r^{*}\left(X_{3}\right)+r^{*}\left(X_{1} \cup X_{3} \cup X_{5}\right)+r^{*}\left(X_{2} \cup X_{3} \cup X_{4}\right)-4 r(M) & +6+5) \\
& +6+9+12+5+5+5+2 \\
=r^{*}\left(X_{3}\right)+r^{*}\left(X_{1} \cup X_{3} \cup X_{5}\right)+r^{*}\left(X_{2} \cup X_{3} \cup X_{4}\right)-4 r(M)+47 & \\
R H S= & r^{*}\left(X_{1} \cup X_{3}\right)+r^{*}\left(X_{1} \cup X_{5}\right)+r^{*}\left(X_{2} \cup X_{3}\right)+r^{*}\left(X_{2} \cup X_{4}\right) \\
& +r^{*}\left(X_{2} \cup X_{5}\right)+r^{*}\left(X_{3} \cup X_{4}\right)+r^{*}\left(X_{4} \cup X_{5}\right) \\
= & r^{*}\left(X_{1} \cup X_{3}\right)+r^{*}\left(X_{2} \cup X_{3}\right)+r^{*}\left(X_{3} \cup X_{4}\right)+\left|X_{1} \cup X_{5}\right| \\
& +\left|X_{2} \cup X_{4}\right|+\left|X_{2} \cup X_{5}\right|+\left|X_{4} \cup X_{5}\right|+r\left(\overline{X_{1} \cup X_{5}}\right) \\
& +r\left(\overline{X_{2} \cup X_{4}}\right)+r\left(\overline{X_{2} \cup X_{5}}\right)+r\left(\overline{X_{4} \cup X_{5}}\right)-4 r(M) \\
\geq & r^{*}\left(X_{1} \cup X_{3}\right)+r^{*}\left(X_{2} \cup X_{3}\right)+r^{*}\left(X_{3} \cup X_{4}\right)-4 r(M) \\
& +9+9+9+9+4+5+4+4 \\
= & r^{*}\left(X_{1} \cup X_{3}\right)+r^{*}\left(X_{2} \cup X_{3}\right)+r^{*}\left(X_{3} \cup X_{4}\right)-4 r(M)+53
\end{aligned}
$$

We must have that

$$
\begin{aligned}
& r^{*}\left(X_{3}\right)+r^{*}\left(X_{1} \cup X_{3} \cup X_{5}\right)+r^{*}\left(X_{2} \cup X_{3} \cup X_{4}\right) \\
> & r^{*}\left(X_{1} \cup X_{3}\right)+r^{*}\left(X_{2} \cup X_{3}\right)+r^{*}\left(X_{3} \cup X_{4}\right)+6
\end{aligned}
$$

Suppose $Z=\varnothing$. Then $2+9+9>8+8+8+6$ which is untrue. The same argument as in subcase 1a shows that if $Z$ is non-empty, we still cannot have a bad family.

Subcase 1.2b: $X_{1}=V_{i} \cup V_{j}$

$X_{2}=V_{i} \cup V_{k}$

$X_{3}=V_{2} \cup Z$ where $Z \subseteq E(M)$

$X_{4}=V_{j} \cup V_{k} \cup\{a\}$ where $a \in V_{1}$

$X_{5}=V_{1}$ 
Compared to subcase 2a, $\left|X_{4}\right|$ increases by one on the left-hand side. On the righthand side, $\left|X_{2} \cup X_{4}\right|$ increases by one. The overall inequality is unchanged, so the same argument as in subcase $2 \mathrm{a}$ holds.

Subcase 1.2c: $X_{1}=V_{i} \cup V_{j}$

$$
\begin{aligned}
& X_{2}=V_{i} \cup V_{k} \\
& X_{3}=V_{2} \cup Z \text { where } Z \subseteq E(M) \\
& X_{4}=V_{j} \cup V_{k} \cup V_{i} \\
& X_{5}=V_{1}
\end{aligned}
$$

Compared to subcase 2a, both $\left|X_{4}\right|$ and $\left|X_{2} \cup X_{4}\right|$ increase by three, leaving the inequality unchanged.

\section{Subcase 1.3.}

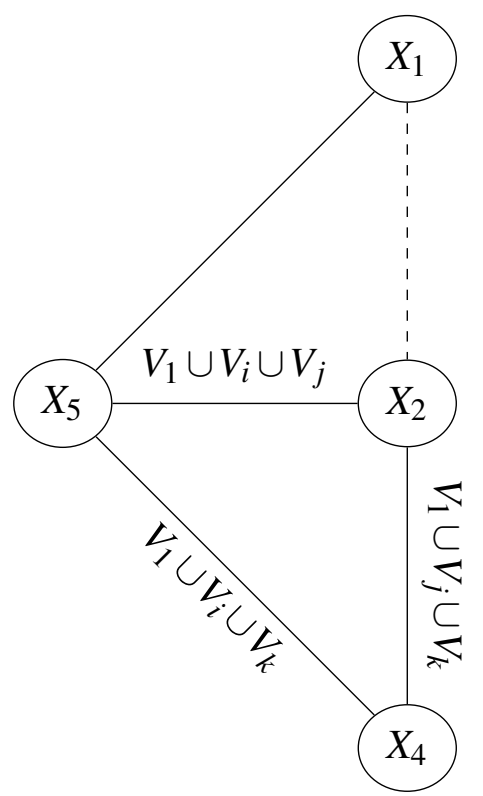

Again, we have by assumption that $V_{2} \subseteq X_{3}$. As $X_{3}$ may contain other elements of the ground set of $M$, any terms in the inequality involving $X_{3}$ will not be immediately evaluated. Recall that $X_{1} \cup X_{2}=V_{3} \cup V_{4} \cup V_{5}$. This means that $V_{1}$ can only be a subset of $X_{4}$ or $X_{5}$. In order for the three circuit-hyperplanes to lie as indicated, both $X_{4}$ and $X_{5}$ must contain $V_{1}$. Also, $X_{2}$ must be equal to $V_{j}$, as this forms the common values of the two circuit-hyperplanes involving $X_{2}$, excluding $V_{1}$. Finally, $X_{5}$ must be equal to $V_{1} \cup V_{i}$, while $X_{4}$ must be equal to $V_{i} \cup V_{k}$. As we assumed that the sets $X_{i}$ are flats, $X_{1}$ could also include one or two elements 
of $V_{j}$. It cannot contain three elements of $V_{j}$, as $V_{i} \cup V_{j} \cup V_{k}$ is a relaxed circuithyperplane in $\left(\operatorname{Kin}(5)^{-}\right)^{*}$ and thus has closure equal to the entire ground set. This gives the following three subcases, which differ only in elements of $X_{1}$. As before, in each subcase we will begin by evaluating terms of the left-hand and right-hand sides of inequality 5 , then show that the left-hand side must be lower in value, meaning that the inequality holds.

Subcase 1.3a: $X_{1}=V_{i} \cup V_{k}$

$$
\begin{aligned}
& X_{2}=V_{j} \\
& X_{3}=V_{2} \cup Z \text { where } Z \subseteq E(M) \\
& X_{4}=V_{1} \cup V_{k} \\
& X_{5}=V_{1} \cup V_{i}
\end{aligned}
$$

Note that $\overline{X_{1} \cup X_{2}}=V_{1} \cup V_{2}$ and $\overline{X_{2} \cup X_{4} \cup X_{5}}=\overline{V_{1} \cup V_{i} \cup V_{j} \cup V_{k}}=V_{2}$.

$$
\begin{aligned}
& \text { LHS }=r^{*}\left(X_{3}\right)+r^{*}\left(X_{4}\right)+r^{*}\left(X_{5}\right)+r^{*}\left(X_{1} \cup X_{2}\right)+r^{*}\left(X_{1} \cup X_{3} \cup X_{5}\right) \\
& +r^{*}\left(X_{2} \cup X_{3} \cup X_{4}\right)+r^{*}\left(X_{2} \cup X_{4} \cup X_{5}\right) \\
& =r^{*}\left(X_{3}\right)+r^{*}\left(X_{1} \cup X_{3} \cup X_{5}\right)+r^{*}\left(X_{2} \cup X_{3} \cup X_{4}\right) \\
& +\left|X_{4}\right|+\left|X_{5}\right|+\left|X_{1} \cup X_{2}\right|+\left|X_{2} \cup X_{4} \cup X_{5}\right| \\
& +r\left(\overline{X_{4}}\right)+r\left(\overline{X_{5}}\right)+r\left(\overline{X_{1} \cup X_{2}}\right)+r\left(\overline{X_{2} \cup X_{4} \cup X_{5}}\right)-4 r(M) \\
& =r^{*}\left(X_{3}\right)+r^{*}\left(X_{1} \cup X_{3} \cup X_{5}\right)+r^{*}\left(X_{2} \cup X_{3} \cup X_{4}\right)-4 r(M) \\
& +6+6+9+12+5+5+5+2 \\
& =r^{*}\left(X_{3}\right)+r^{*}\left(X_{1} \cup X_{3} \cup X_{5}\right)+r^{*}\left(X_{2} \cup X_{3} \cup X_{4}\right)-4 r(M)+50 \\
& R H S=r^{*}\left(X_{1} \cup X_{3}\right)+r^{*}\left(X_{1} \cup X_{5}\right)+r^{*}\left(X_{2} \cup X_{3}\right)+r^{*}\left(X_{2} \cup X_{4}\right) \\
& +r^{*}\left(X_{2} \cup X_{5}\right)+r^{*}\left(X_{3} \cup X_{4}\right)+r^{*}\left(X_{4} \cup X_{5}\right) \\
& =r^{*}\left(X_{1} \cup X_{3}\right)+r^{*}\left(X_{2} \cup X_{3}\right)+r^{*}\left(X_{3} \cup X_{4}\right)+\left|X_{1} \cup X_{5}\right| \\
& +\left|X_{2} \cup X_{4}\right|+\left|X_{2} \cup X_{5}\right|+\left|X_{4} \cup X_{5}\right|+r\left(\overline{X_{1} \cup X_{5}}\right) \\
& +r\left(\overline{X_{2} \cup X_{4}}\right)+r\left(\overline{X_{2} \cup X_{5}}\right)+r\left(\overline{X_{4} \cup X_{5}}\right)-4 r(M) \\
& \geq r^{*}\left(X_{1} \cup X_{3}\right)+r^{*}\left(X_{2} \cup X_{3}\right)+r^{*}\left(X_{3} \cup X_{4}\right)-4 r(M) \\
& +9+9+9+9+4+4+4+4 \\
& =r^{*}\left(X_{1} \cup X_{3}\right)+r^{*}\left(X_{2} \cup X_{3}\right)+r^{*}\left(X_{3} \cup X_{4}\right)-4 r(M)+52
\end{aligned}
$$


We must have that

$$
\begin{aligned}
& r^{*}\left(X_{3}\right)+r^{*}\left(X_{1} \cup X_{3} \cup X_{5}\right)+r^{*}\left(X_{2} \cup X_{3} \cup X_{4}\right) \\
> & r^{*}\left(X_{1} \cup X_{3}\right)+r^{*}\left(X_{2} \cup X_{3}\right)+r^{*}\left(X_{3} \cup X_{4}\right)+2
\end{aligned}
$$

Suppose $Z=\varnothing$. Then we have $2+9+9>8+5+8+2$ which is untrue. If we increase the size of $Z$, the same argument as in subcase 1a shows that we still cannot have a bad family.

Subcase 1.3b: $X_{1}=V_{i} \cup V_{k} \cup\{a\}$ where $a \in V_{j}$

$$
\begin{aligned}
& X_{2}=V_{j} \\
& X_{3}=V_{2} \cup Z \text { where } Z \subseteq E(M) \\
& X_{4}=V_{1} \cup V_{k} \\
& X_{5}=V_{1} \cup V_{i}
\end{aligned}
$$

Compared to subcase $3 \mathrm{a},\left|X_{1} \cup X_{5}\right|$ increases by one, and the same argument still holds.

Subcase 1.3c: $X_{1}=V_{i} \cup V_{k} \cup\{a, b\}$ where $a, b \in V_{j}$

$$
\begin{aligned}
& X_{2}=V_{j} \\
& X_{3}=V_{2} \cup Z \text { where } Z \subseteq E(M) \\
& X_{4}=V_{1} \cup V_{k} \\
& X_{5}=V_{1} \cup V_{i}
\end{aligned}
$$

Compared to subcase 3a, $\left|X_{1} \cup X_{5}\right|$ increases by two, while $r\left(\overline{X_{1} \cup X_{5}}\right)$ falls by one. The right-hand side of the inequality increases by one, and the same argument as before holds. 


\section{Subcase 1.4.}

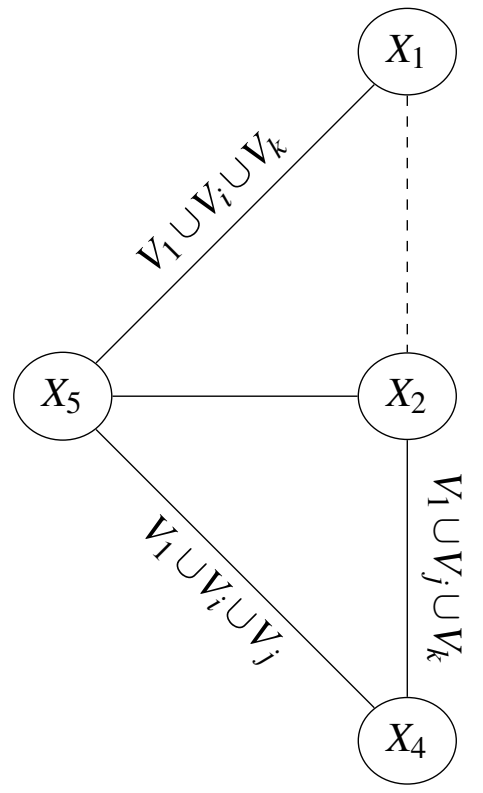

Again, we have by assumption that $V_{2} \subseteq X_{3}$. As $X_{3}$ may contain other elements of the ground set of $M$, any terms in the inequality involving $X_{3}$ will not be immediately evaluated. Recall that $X_{1} \cup X_{2}=V_{3} \cup V_{4} \cup V_{5}$. This means that $V_{1}$ can only be a subset of $X_{4}$ or $X_{5}$. In order for the three circuit-hyperplanes to lie as indicated, both $X_{4}$ and $X_{5}$ must contain $V_{1}$. As $X_{2} \cup X_{4}$ cannot contain $V_{i}, X_{5}$ must be equal to $V_{1} \cup V_{i}$. Likewise, $X_{2}$ must contain $V_{k}$. This forces $X_{4}$ to contain $V_{j}$, and $X_{1}$ to contain $V_{k}$. Finally, in order for $X_{1} \cup X_{2}=V_{3} \cup V_{4} \cup V_{5}$, it must be that $X_{2}$ is equal to $V_{j} \cup V_{k}$ and $X_{1}$ is equal to $V_{i} \cup V_{k}$.

$$
\begin{aligned}
& X_{1}=V_{i} \cup V_{k} \\
& X_{2}=V_{j} \cup V_{k} \\
& X_{3}=V_{2} \cup Z \text { where } Z \subseteq E(M) \\
& X_{4}=V_{1} \cup V_{j} \\
& X_{5}=V_{1} \cup V i
\end{aligned}
$$


Note that $\overline{X_{1} \cup X_{2}}=V_{1} \cup V_{2}$ and $\overline{X_{2} \cup X_{4} \cup X_{5}}=\overline{V_{1} \cup V_{i} \cup V_{j} \cup V_{k}}=V_{2}$.

$$
\begin{aligned}
\text { LHS }= & r^{*}\left(X_{3}\right)+r^{*}\left(X_{4}\right)+r^{*}\left(X_{5}\right)+r^{*}\left(X_{1} \cup X_{2}\right)+r^{*}\left(X_{1} \cup X_{3} \cup X_{5}\right) \\
& +r^{*}\left(X_{2} \cup X_{3} \cup X_{4}\right)+r^{*}\left(X_{2} \cup X_{4} \cup X_{5}\right) \\
= & r^{*}\left(X_{3}\right)+r^{*}\left(X_{1} \cup X_{3} \cup X_{5}\right)+r^{*}\left(X_{2} \cup X_{3} \cup X_{4}\right) \\
& +\left|X_{4}\right|+\left|X_{5}\right|+\left|X_{1} \cup X_{2}\right|+\left|X_{2} \cup X_{4} \cup X_{5}\right| \\
& +r\left(\overline{X_{4}}\right)+r\left(\overline{X_{5}}\right)+r\left(\overline{X_{1} \cup X_{2}}\right)+r\left(\overline{X_{2} \cup X_{4} \cup X_{5}}\right)-4 r(M) \\
=r^{*}\left(X_{3}\right)+r^{*}\left(X_{1} \cup X_{3} \cup X_{5}\right)+r^{*}\left(X_{2} \cup X_{3} \cup X_{4}\right)-4 r(M) & +6+6+9+12+5+5+5+2 \\
& +6+X^{*}\left(X_{3}\right)+r^{*}\left(X_{1} \cup X_{3} \cup X_{5}\right)+r^{*}\left(X_{2} \cup X_{3} \cup X_{4}\right)-4 r(M)+50 \\
R H S= & r^{*}\left(X_{1} \cup X_{3}\right)+r^{*}\left(X_{1} \cup X_{5}\right)+r^{*}\left(X_{2} \cup X_{3}\right)+r^{*}\left(X_{2} \cup X_{4}\right) \\
& +r^{*}\left(X_{2} \cup X_{5}\right)+r^{*}\left(X_{3} \cup X_{4}\right)+r^{*}\left(X_{4} \cup X_{5}\right) \\
= & r^{*}\left(X_{1} \cup X_{3}\right)+r^{*}\left(X_{2} \cup X_{3}\right)+r^{*}\left(X_{3} \cup X_{4}\right)+\left|X_{1} \cup X_{5}\right| \\
& +\left|X_{2} \cup X_{4}\right|+\left|X_{2} \cup X_{5}\right|+\left|X_{4} \cup X_{5}\right|+r\left(\overline{X_{1} \cup X_{5}}\right) \\
& +r\left(\overline{X_{2} \cup X_{4}}\right)+r\left(\overline{X_{2} \cup X_{5}}\right)+r\left(\overline{X_{4} \cup X_{5}}\right)-4 r(M) \\
\geq & r^{*}\left(X_{1} \cup X_{3}\right)+r^{*}\left(X_{2} \cup X_{3}\right)+r^{*}\left(X_{3} \cup X_{4}\right)-4 r(M) \\
& +9+9+9+9+4+4+2+4 \\
= & r^{*}\left(X_{1} \cup X_{3}\right)+r^{*}\left(X_{2} \cup X_{3}\right)+r^{*}\left(X_{3} \cup X_{4}\right)-4 r(M)+50
\end{aligned}
$$

We must have that

$$
\begin{aligned}
& r^{*}\left(X_{3}\right)+r^{*}\left(X_{1} \cup X_{3} \cup X_{5}\right)+r^{*}\left(X_{2} \cup X_{3} \cup X_{4}\right) \\
> & r^{*}\left(X_{1} \cup X_{3}\right)+r^{*}\left(X_{2} \cup X_{3}\right)+r^{*}\left(X_{3} \cup X_{4}\right)
\end{aligned}
$$

Suppose $Z=\varnothing$. Then we have $2+9+9>8+8+8$ which is untrue. If we increase the size of $Z$, the same argument as in subcase 1a shows that we still cannot have a bad family.

\section{Case 2. $X_{4} \cap V_{2} \neq \varnothing$}

As in Case 1, we will consider which term on the left-hand side dual inequality must be $V_{3} \cup V_{4} \cup V_{5}$. As this term does not contain any elements of $V_{2}$ and these elements are contained in $X_{4}$, the term cannot involve $X_{4}$. We now have the following representation of the locations remaining as possibilities for all necessary 
circuit-hyperplanes, where the dashed line again indicates a term which falls on the left-hand side of the inequality.
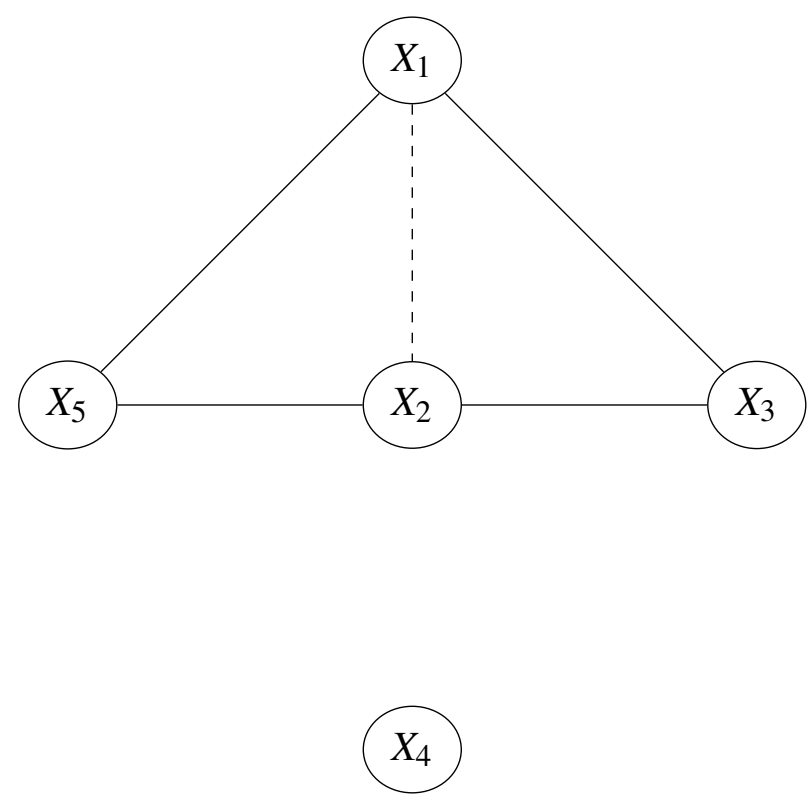

If $V_{3} \cup V_{4} \cup V_{5}$ is equal to $X_{3}$ or $X_{5}$ then any term involving these sets cannot be one of the circuit-hyperplanes $V_{1} \cup X_{4} \cup V_{5}$ or $V_{1} \cup V_{3} \cup V_{5}$. When $V_{3} \cup V_{4} \cup V_{5}=X_{3}$, the possible terms left are $X_{1} \cup X_{5}$ and $X_{2} \cup X_{5}$. When $V_{3} \cup V_{4} \cup V_{5}=X_{5}$, the possible terms left are $X_{1} \cup X_{3}$ and $X_{2} \cup X_{3}$. In both cases there are not enough terms on the right-hand side for the three circuit-hyperplanes. Thus $V_{3} \cup V_{4} \cup V_{5}=X_{1} \cup X_{2}$. As $X_{3}$ and $X_{5}$ can be switched with no effect on the inequality, we now have, up to symmetry, two possibilities of where the circuit-hyperplanes lie, as indicated by the bold lines in the following diagrams.
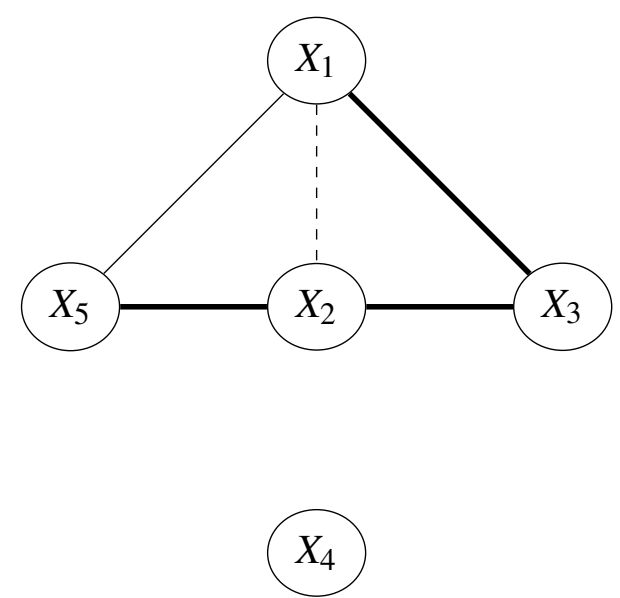

Subcase 2.1
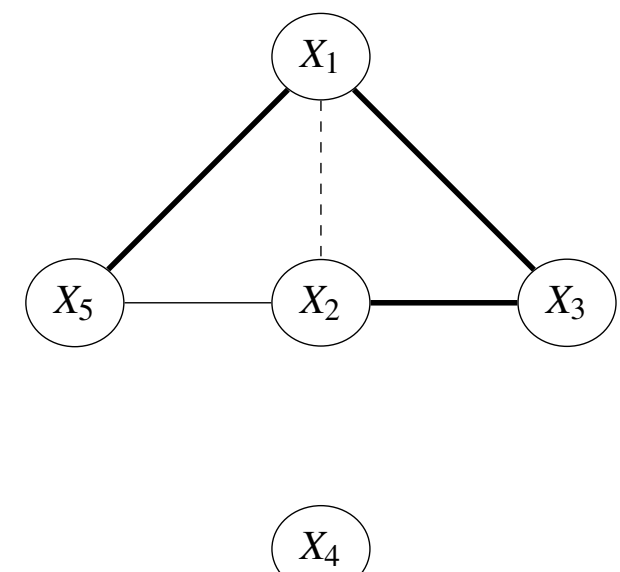

Subcase 2.2 
We will again consider both of these subcases, with circuit-hyperplanes lying as shown in the diagrams, which give multiple options within each subcase for the choice of sets $X_{1}, \ldots, X_{5}$.

\section{Subcase 2.1.}
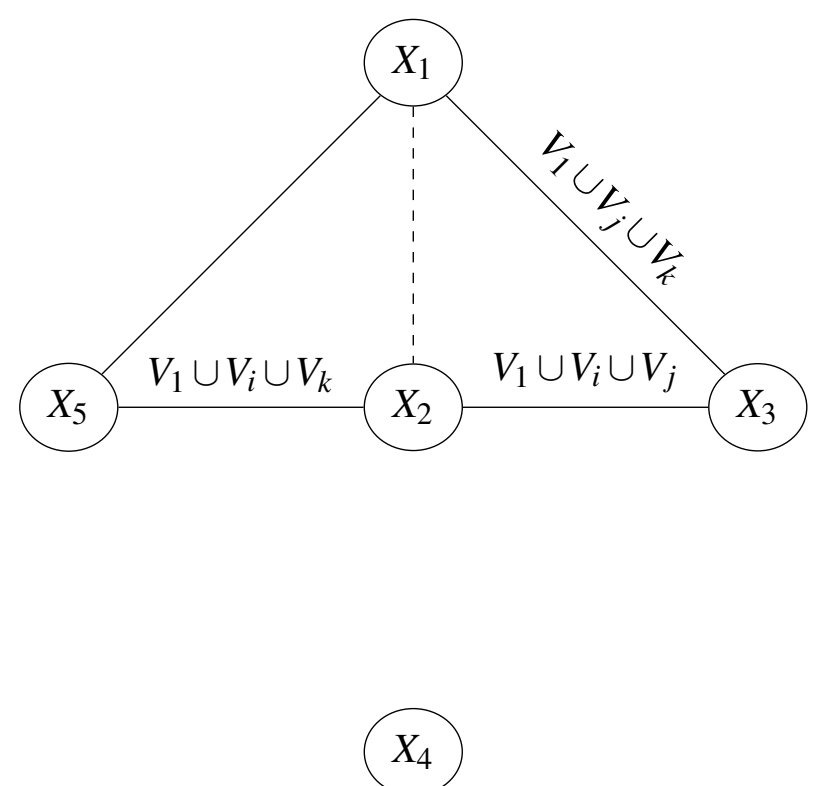

Here, we have assumed that $V_{2} \subseteq X_{4}$. As $X_{4}$ may contain other elements of the ground set of $M$, any terms in the inequality involving $X_{4}$ will not be immediately evaluated. Recall that $X_{1} \cup X_{2}=V_{3} \cup V_{4} \cup V_{5}$. This means that $V_{1}$ can only be a subset of $X_{3}$ or $X_{5}$. In order for the three circuit-hyperplanes to lie as indicated, both $X_{3}$ and $X_{5}$ must contain $V_{1}$. Note $X_{2}$ must be equal to $V_{i}$, as this forms the common values of the two circuit-hyperplanes involving $X_{2}$, excluding $V_{1}$. This forces $X_{1}$ to be equal to $V_{j} \cup V_{k}$. Note that $X_{1}$ cannot contain any elements of $V_{i}$ as $X_{1} \cup X_{3}$ does not. Also, $X_{3}$ must contain $V_{j}$ and $X_{5}$ must contain $V_{k}$. We have that $X_{3}$ must be equal to $V_{1} \cup V_{j}$. As we assumed that the sets $X_{i}$ are flats, $X_{5}$ could also include one or three elements of $V_{i} . X_{5}$ cannot contain exactly two elements of $V_{i}$ as $V_{1} \cup V_{k} \cup V_{i}$ is a circuit-hyperplane in the dual.This gives the following three subcases, which differ only in elements of $X_{5}$.

Subcase 2.1a: $X_{1}=V_{j} \cup V_{k}$

$$
\begin{aligned}
& X_{2}=V_{i} \\
& X_{3}=V_{1} \cup V_{j} \\
& X_{4}=V_{2} \cup Z \text { where } Z \subseteq E(M) \\
& X_{5}=V_{1} \cup V_{k}
\end{aligned}
$$


Note that $\overline{X_{1} \cup X_{2}}=V_{1} \cup V_{2}$ and $\overline{X_{1} \cup X_{3} \cup X_{5}}=\overline{V_{1} \cup V_{j} \cup V_{k}}=V_{2} \cup V_{i}$.

$$
\begin{aligned}
\text { LHS }= & r^{*}\left(X_{3}\right)+r^{*}\left(X_{4}\right)+r^{*}\left(X_{5}\right)+r^{*}\left(X_{1} \cup X_{2}\right)+r^{*}\left(X_{1} \cup X_{3} \cup X_{5}\right) \\
& +r^{*}\left(X_{2} \cup X_{3} \cup X_{4}\right)+r^{*}\left(X_{2} \cup X_{4} \cup X_{5}\right) \\
=r^{*} & \left(X_{4}\right)+r^{*}\left(X_{2} \cup X_{3} \cup X_{4}\right)+r^{*}\left(X_{2} \cup X_{4} \cup X_{5}\right) \\
& +\left|X_{3}\right|+\left|X_{5}\right|+\left|X_{1} \cup X_{2}\right|+\left|X_{1} \cup X_{3} \cup X_{5}\right| \\
& +r\left(\overline{X_{3}}\right)+r\left(\overline{X_{5}}\right)+r\left(\overline{X_{1} \cup X_{2}}\right)+r\left(\overline{X_{1} \cup X_{3} \cup X_{5}}\right)-4 r(M) \\
=r^{*}( & \left.X_{4}\right)+r^{*}\left(X_{2} \cup X_{3} \cup X_{4}\right)+r^{*}\left(X_{2} \cup X_{4} \cup X_{5}\right)-4 r(M) \\
& +6+6+9+9+5+5+5+4 \\
=r^{*}\left(X_{4}\right)+r^{*}\left(X_{2} \cup X_{3} \cup X_{4}\right)+r^{*}\left(X_{2} \cup X_{4} \cup X_{5}\right)-4 r(M)+49 & \\
R H S= & r^{*}\left(X_{1} \cup X_{3}\right)+r^{*}\left(X_{1} \cup X_{5}\right)+r^{*}\left(X_{2} \cup X_{3}\right)+r^{*}\left(X_{2} \cup X_{4}\right) \\
& +r^{*}\left(X_{2} \cup X_{5}\right)+r^{*}\left(X_{3} \cup X_{4}\right)+r^{*}\left(X_{4} \cup X_{5}\right) \\
= & r^{*}\left(X_{2} \cup X_{4}\right)+r^{*}\left(X_{3} \cup X_{4}\right)+r^{*}\left(X_{4} \cup X_{5}\right)+\left|X_{1} \cup X_{3}\right| \\
& +\left|X_{1} \cup X_{5}\right|+\left|X_{2} \cup X_{3}\right|+\left|X_{2} \cup X_{5}\right|+r\left(\overline{X_{1} \cup X_{3}}\right) \\
& +r\left(\overline{X_{1} \cup X_{5}}\right)+r\left(\overline{X_{2} \cup X_{3}}\right)+r\left(\overline{X_{2} \cup X_{5}}\right)-4 r(M) \\
= & r^{*}\left(X_{2} \cup X_{4}\right)+r^{*}\left(X_{3} \cup X_{4}\right)+r^{*}\left(X_{4} \cup X_{5}\right)-4 r(M) \\
& +9+9+9+9+4+4+4+4 \\
= & r^{*}\left(X_{2} \cup X_{4}\right)+r^{*}\left(X_{3} \cup X_{4}\right)+r^{*}\left(X_{4} \cup X_{5}\right)-4 r(M)+52
\end{aligned}
$$

We must have that

$$
\begin{aligned}
& r^{*}\left(X_{4}\right)+r^{*}\left(X_{2} \cup X_{3} \cup X_{4}\right)+r^{*}\left(X_{2} \cup X_{4} \cup X_{5}\right) \\
> & r^{*}\left(X_{2} \cup X_{4}\right)+r^{*}\left(X_{3} \cup X_{4}\right)+r^{*}\left(X_{4} \cup X_{5}\right)+3
\end{aligned}
$$

Suppose $Z=\varnothing$. Then we have that $2+8+8>5+8+8+3$ which is untrue. Now suppose $Z$ is non-empty. Note that $X_{2} \cup X_{3} \cup X_{4}=V_{1} \cup V_{2} \cup V_{i} \cup V_{j} \cup Z$ and $\overline{X_{2} \cup X_{3} \cup X_{4}}=V_{k}-Z$, so if $\left|X_{2} \cup X_{3} \cup X_{4}\right|$ increases, then $Z \subseteq V_{k}$, and $r\left(\overline{X_{2} \cup X_{3} \cup X_{4}}\right)$ must decrease by the same amount. Thus $r^{*}\left(X_{2} \cup X_{3} \cup X_{4}\right)$ remains unchanged. Likewise, $r^{*}\left(X_{2} \cup X_{4} \cup X_{5}\right)$ cannot change, as $X_{2} \cup X_{4} \cup X_{5}=$ $V_{1} \cup V_{2} \cup V_{i} \cup V_{j} \cup Z$ and has complement $V_{k}-Z$. Finally, $r^{*}\left(X_{4}\right)$ must be no greater than $r^{*}\left(X_{4} \cup X_{5}\right)$. We thus do not have a bad family.

Subcase 2.1b: $X_{1}=V_{j} \cup V_{k}$

$X_{2}=V_{i}$ 


$$
\begin{aligned}
& X_{3}=V_{1} \cup V_{j} \\
& X_{4}=V_{2} \cup Z \text { where } Z \subseteq E(M) \\
& X_{5}=V_{1} \cup V_{k} \cup\{a\} \text { where } a \in V_{i}
\end{aligned}
$$

On the left-hand side, $\left|X_{5}\right|$ and $\left|X_{1} \cup X_{3} \cup X_{5}\right|$ both increase in size by two. On the right-hand side, $\left|X_{1} \cup X_{5}\right|$ increases in size by one. The same argument as in Subcase 1a shows there can be no bad family regardless of $Z$.

Subcase 2.1c: $X_{1}=V_{j} \cup V_{k}$

$$
\begin{aligned}
& X_{2}=V_{i} \\
& X_{3}=V_{1} \cup V_{j} \\
& X_{4}=V_{2} \cup Z \text { where } Z \subseteq E(M) \\
& X_{5}=V_{1} \cup V_{k} \cup\{a, b\} \text { where } a, b \in V_{i}
\end{aligned}
$$

On the left-hand side, $\left|X_{5}\right|$ and $\left|X_{1} \cup X_{3} \cup X_{5}\right|$ both increase in size by two, while $r\left(X_{1} \cup X_{3} \cup X_{5}\right)$ falls by one. On the right-hand side, $\left|X_{1} \cup X_{5}\right|$ increases in size by two while $r\left(X_{1} \cup X_{5}\right)$ falls by one. We still have no bad family.

\section{Subcase 2.2.}
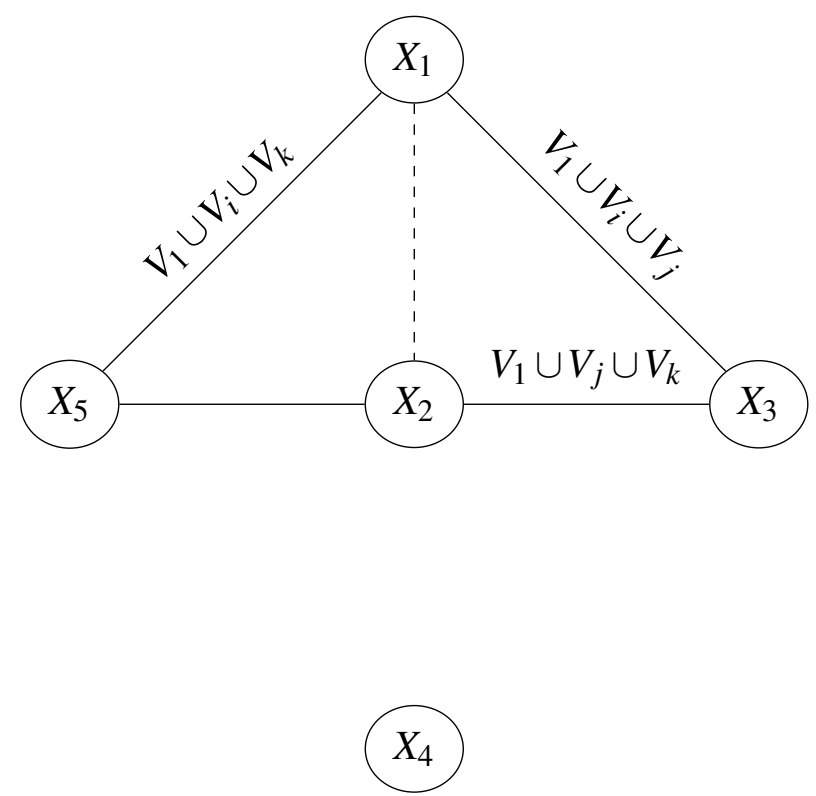

Again, we have assumed that $V_{2} \subseteq X_{4}$. As $X_{4}$ may contain other elements of the ground set of $M$, any terms in the inequality involving $X_{4}$ will not be immediately evaluated. Recall that $X_{1} \cup X_{2}=V_{3} \cup V_{4} \cup V_{5}$. This means that $V_{1}$ can only be a subset of $X_{3}$ or $X_{5}$. In order for the three circuit-hyperplanes to lie as indicated, 
both $X_{3}$ and $X_{5}$ must contain $V_{1}$. Also, $X_{1}$ must be equal to $V_{i}$, as this forms the common values of the two circuit-hyperplanes involving $X_{2}$, excluding $V_{1}$. This forces $X_{2}$ to be equal to $V_{j} \cup V_{k}$, forces $X_{3}$ to be equal to $V_{1} \cup V_{j}$, and forces $X_{5}$ to contain $V_{1} \cup V_{k}$. As we assumed that the sets $X_{i}$ are flats, $X_{5}$ could also include one or three elements of $V_{i}$. Note $X_{5}$ cannot contain exactly two elements of $V_{i}$ as $V_{1} \cup V_{k} \cup V_{i}$ is a circuit-hyperplane in the dual.This gives the following three subcases, which differ only in elements of $X_{5}$.

Subcase 2.2a: $X_{1}=V_{i}$

$$
\begin{aligned}
& X_{2}=V_{j} \cup V_{k} \\
& X_{3}=V_{1} \cup V_{j} \\
& X_{4}=V_{2} \cup Z \text { where } Z \subseteq E(M) \\
& X_{5}=V_{1} \cup V_{k}
\end{aligned}
$$

Note that $\overline{X_{1} \cup X_{2}}=V_{1} \cup V_{2}$ and $\overline{X_{1} \cup X_{3} \cup X_{5}}=\overline{V_{1} \cup V_{i} \cup V_{j} \cup V_{k}}=V_{2}$.

$$
\begin{aligned}
& \text { LHS }=r^{*}\left(X_{3}\right)+r^{*}\left(X_{4}\right)+r^{*}\left(X_{5}\right)+r^{*}\left(X_{1} \cup X_{2}\right)+r^{*}\left(X_{1} \cup X_{3} \cup X_{5}\right) \\
& +r^{*}\left(X_{2} \cup X_{3} \cup X_{4}\right)+r^{*}\left(X_{2} \cup X_{4} \cup X_{5}\right) \\
& =r^{*}\left(X_{4}\right)+r^{*}\left(X_{2} \cup X_{3} \cup X_{4}\right)+r^{*}\left(X_{2} \cup X_{4} \cup X_{5}\right) \\
& +\left|X_{3}\right|+\left|X_{5}\right|+\left|X_{1} \cup X_{2}\right|+\left|X_{1} \cup X_{3} \cup X_{5}\right| \\
& +r\left(\overline{X_{3}}\right)+r\left(\overline{X_{5}}\right)+r\left(\overline{X_{1} \cup X_{2}}\right)+r\left(\overline{X_{1} \cup X_{3} \cup X_{5}}\right)-4 r(M) \\
& =r^{*}\left(X_{4}\right)+r^{*}\left(X_{2} \cup X_{3} \cup X_{4}\right)+r^{*}\left(X_{2} \cup X_{4} \cup X_{5}\right)-4 r(M) \\
& +6+6+9+12+5+5+5+2 \\
& =r^{*}\left(X_{4}\right)+r^{*}\left(X_{2} \cup X_{3} \cup X_{4}\right)+r^{*}\left(X_{2} \cup X_{4} \cup X_{5}\right)-4 r(M)+50 \\
& R H S=r^{*}\left(X_{1} \cup X_{3}\right)+r^{*}\left(X_{1} \cup X_{5}\right)+r^{*}\left(X_{2} \cup X_{3}\right)+r^{*}\left(X_{2} \cup X_{4}\right) \\
& +r^{*}\left(X_{2} \cup X_{5}\right)+r^{*}\left(X_{3} \cup X_{4}\right)+r^{*}\left(X_{4} \cup X_{5}\right) \\
& =r^{*}\left(X_{2} \cup X_{4}\right)+r^{*}\left(X_{3} \cup X_{4}\right)+r^{*}\left(X_{4} \cup X_{5}\right)+\left|X_{1} \cup X_{3}\right| \\
& +\left|X_{1} \cup X_{5}\right|+\left|X_{2} \cup X_{3}\right|+\left|X_{2} \cup X_{5}\right|+r\left(\overline{X_{1} \cup X_{3}}\right) \\
& +r\left(\overline{X_{1} \cup X_{5}}\right)+r\left(\overline{X_{2} \cup X_{3}}\right)+r\left(\overline{X_{2} \cup X_{5}}\right)-4 r(M) \\
& =r^{*}\left(X_{2} \cup X_{4}\right)+r^{*}\left(X_{3} \cup X_{4}\right)+r^{*}\left(X_{4} \cup X_{5}\right)-4 r(M) \\
& +9+9+9+9+4+4+4+4 \\
& =r^{*}\left(X_{2} \cup X_{4}\right)+r^{*}\left(X_{3} \cup X_{4}\right)+r^{*}\left(X_{4} \cup X_{5}\right)-4 r(M)+52
\end{aligned}
$$


We must have that

$$
\begin{array}{r}
r^{*}\left(X_{4}\right)+r^{*}\left(X_{2} \cup X_{3} \cup X_{4}\right)+r^{*}\left(X_{2} \cup X_{4} \cup X_{5}\right) \\
>r^{*}\left(X_{2} \cup X_{4}\right)+r^{*}\left(X_{3} \cup X_{4}\right)+r^{*}\left(X_{4} \cup X_{5}\right)+2
\end{array}
$$

Suppose $Z=\varnothing$. Then we have that $2+8+8>8+8+8+2$ which is untrue. Now suppose $Z$ is non-empty. The same argument from Subcase 1a shows we still cannot have a bad family.

Subcase 2.2b: $X_{1}=V_{i}$

$$
\begin{aligned}
& X_{2}=V_{j} \cup V_{k} \\
& X_{3}=V_{1} \cup V_{j} \\
& X_{4}=V_{2} \cup Z \text { where } Z \subseteq E(M) \\
& X_{5}=V_{1} \cup V_{k} \cup\{a\} \text { where } a \in V_{i}
\end{aligned}
$$

On the left-hand side, $\left|X_{5}\right|$ increases by one relative to Subcase 2.2a. On the righthand side, $\left|X_{2} \cup X_{5}\right|$ increases by one. We still have no bad family.

Subcase 2.2c: $X_{1}=V_{i}$

$$
\begin{aligned}
& X_{2}=V_{j} \cup V_{k} \\
& X_{3}=V_{1} \cup V_{j} \\
& X_{4}=V_{2} \cup Z \text { where } Z \subseteq E(M) \\
& X_{5}=V_{1} \cup V_{k} \cup\{a, b\} \text { where } a, b \in V_{i}
\end{aligned}
$$

Both $\left|X_{5}\right|$ and $\left|X_{2} \cup X_{5}\right|$ increase by two, while $r\left(\overline{X_{2} \cup X_{5}}\right)$ falls by one. We still have no bad family.

Case 3. $X_{2} \cap V_{2} \neq \varnothing$

Again consider which term on the left-hand side dual inequality must be $V_{3} \cup$ $V_{4} \cup V_{5}$. As this term does not contain any elements of $V_{2}$ and these elements are contained in $X_{2}$, the term cannot involve $X_{2}$. We have the following representation of remaining possible locations for the necessary circuit-hyperplanes. 


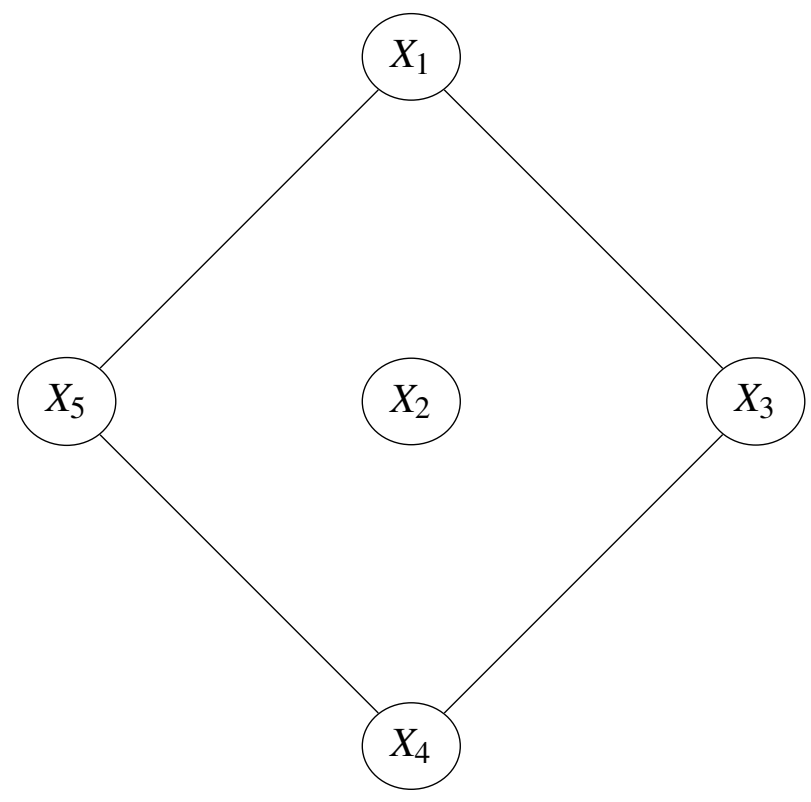

If $V_{3} \cup V_{4} \cup V_{5}$ is equal to $X_{3}, X_{4}$, or $X_{5}$ then any term involving these sets cannot be one of the circuit-hyperplanes $V_{1} \cup V_{3} \cup V_{4}, V_{1} \cup X_{4} \cup V_{5}$, or $V_{1} \cup V_{3} \cup V_{5}$. This will leave us with two terms on the right-hand side which could be the circuithyperplanes, but three are needed. If it is equal to $X_{1} \cup X_{3} \cup X_{5}$, then only $X_{4}$ can contain elements of $V_{1}$. As $V_{1}$ appears in all of the three circuit-hyperplanes needed on the right-hand side of the inequality, and there are only two terms available using $X_{4}$, we do not have enough terms left for the circuit-hyperplanes. This covers all terms on the left-hand side, and thus $X_{2} \cup V_{2}=\varnothing$.

Case 4. $X_{1} \cap V_{2} \neq \varnothing$

Consider which term on the left-hand side of the dual inequality must be $V_{3} \cup V_{4} \cup$ $V_{5}$. It cannot be any of the terms involving $X_{1}$. We have the following representation of the remaining possible locations for the necessary circuit-hyperplanes. 

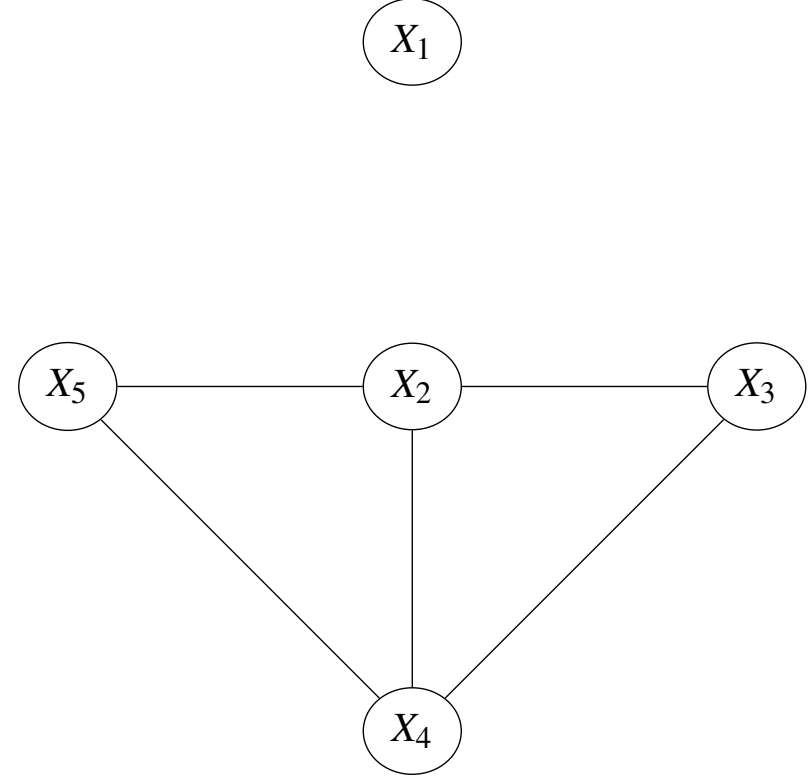

If $V_{3} \cup V_{4} \cup V_{5}$ is $X_{1} \cup X_{3} \cup X_{5}, X_{2} \cup X_{3} \cup X_{4}$, or $X_{2} \cup X_{4} \cup X_{5}$, only two sets could include elements of $V_{1}$. We will not have enough terms left on the right-hand side to be the circuit-hyperplanes $V_{1} \cup V_{3} \cup V_{4}, V_{1} \cup X_{4} \cup V_{5}$, and $V_{1} \cup V_{3} \cup V_{5}$. If $V_{3} \cup V_{4} \cup V_{5}=X_{4}$, the circuit-hyperplanes cannot be terms using $X_{4}$, so the only possible edges remaining are $X_{2} \cup X_{3}$ and $X_{2} \cup X_{5}$ - one edge less than is necessary. Thus $V_{3} \cup V_{4} \cup V_{5}$ must be either $X_{3}$ or $X_{5}$. These two possibilities are symmetric, so assume $V_{3} \cup V_{4} \cup V_{5}=X_{3}$. The necessary circuit-hyperplanes now cannot use $X_{3}$ or $X_{1}$. This gives only one possible assignment to the terms in the inequality, as shown in the following diagram.

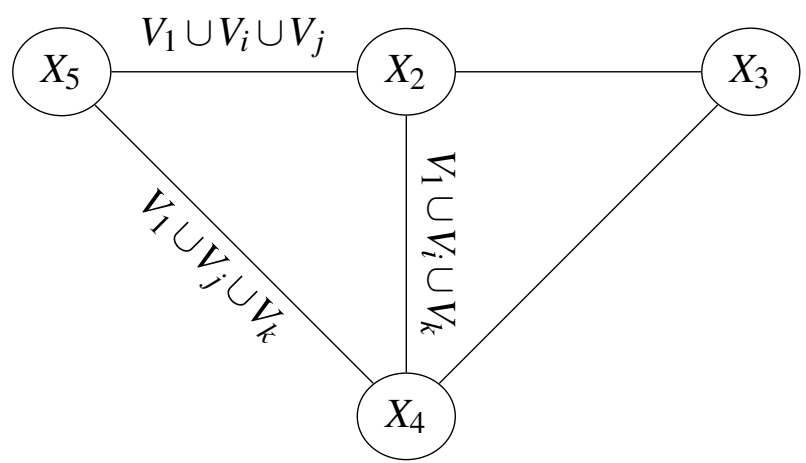


We have that $X_{1}=V_{2} \cup Z$ where $Z$ is a possibly empty subset of the ground set and that $X_{3}=V_{3} \cup V_{4} \cup V_{5} . X_{2}$ must contain $V_{i}$ but cannot contain $V_{j}$ or $V_{k}, X_{4}$ must contain $V_{k}$ but cannot contain $V_{i}$ or $V_{j}$, and $X_{5}$ must contain $V_{j}$ but cannot contain $V_{i}$ or $V_{k}$. All three of these sets could also contain one of three elements from $V_{1}$, such that whenever we take the union of two of the sets, the union contains $V_{1}$. A set cannot contain two elements of $V_{1}$ as this would mean it was not a flat.

Note that if one of the sets $X_{2}, X_{4}$, or $X_{5}$ contains no elements of $V_{1}$, both the other two sets must contain all elements of $V_{1}$. If one of the sets contains one element of $V_{1}$, the other two sets must again contain all elements of $V_{1}$. The third possibility is that all three sets contain $V_{1}$.

Subcase 4.1a: $X_{1}=V_{2} \cup Z$ where $Z \subseteq E(M)$

$$
\begin{aligned}
& X_{2}=V_{i} \cup V_{1} \\
& X_{3}=V_{3} \cup V_{4} \cup V_{5} \\
& X_{4}=V_{k} \\
& X_{5}=V_{j} \cup V_{1}
\end{aligned}
$$

Note that $\overline{X_{2} \cup X_{3} \cup X_{4}}=\overline{X_{2} \cup X_{4} \cup X_{5}}=\overline{V_{1} \cup V_{i} \cup V_{j} \cup V_{k}}=V_{2}$.

$$
\begin{aligned}
\text { LHS }=r^{*} & \left(X_{3}\right)+r^{*}\left(X_{4}\right)+r^{*}\left(X_{5}\right)+r^{*}\left(X_{1} \cup X_{2}\right)+r^{*}\left(X_{1} \cup X_{3} \cup X_{5}\right) \\
& +r^{*}\left(X_{2} \cup X_{3} \cup X_{4}\right)+r^{*}\left(X_{2} \cup X_{4} \cup X_{5}\right) \\
=r^{*} & \left(X_{1} \cup X_{2}\right)+r^{*}\left(X_{1} \cup X_{3} \cup X_{5}\right)+\left|X_{3}\right|+\left|X_{4}\right|+\left|X_{5}\right| \\
& +\left|X_{2} \cup X_{3} \cup X_{4}\right|+\left|X_{2} \cup X_{4} \cup X_{5}\right|+r\left(\overline{X_{3}}\right)+r\left(\overline{X_{4}}\right) \\
& +r\left(\overline{X_{5}}\right)+r\left(\overline{X_{2} \cup X_{3} \cup X_{4}}\right)+r\left(\overline{X_{2} \cup X_{4} \cup X_{5}}\right)-5 r(M) \\
=r^{*} & \left(X_{1} \cup X_{2}\right)+r^{*}\left(X_{1} \cup X_{3} \cup X_{5}\right)-5 r(M)+9+3+6 \\
& +12+12+5+5+5+2+2 \\
= & r^{*}\left(X_{1} \cup X_{2}\right)+r^{*}\left(X_{1} \cup X_{3} \cup X_{5}\right)-5 r(M)+61
\end{aligned}
$$




$$
\begin{aligned}
R H S=r^{*} & \left(X_{1} \cup X_{3}\right)+r^{*}\left(X_{1} \cup X_{5}\right)+r^{*}\left(X_{2} \cup X_{3}\right)+r^{*}\left(X_{2} \cup X_{4}\right) \\
& +r^{*}\left(X_{2} \cup X_{5}\right)+r^{*}\left(X_{3} \cup X_{4}\right)+r^{*}\left(X_{4} \cup X_{5}\right) \\
=r^{*} & \left(X_{1} \cup X_{3}\right)+r^{*}\left(X_{1} \cup X_{5}\right)+\left|X_{2} \cup X_{3}\right|+\left|X_{2} \cup X_{4}\right|+\left|X_{2} \cup X_{5}\right| \\
& +\left|X_{3} \cup X_{4}\right|+\left|X_{4} \cup X_{5}\right|+r\left(\overline{X_{2} \cup X_{3}}\right)+r\left(\overline{X_{2} \cup X_{4}}\right) \\
& +r\left(\overline{X_{2} \cup X_{5}}\right)+r\left(\overline{X_{3} \cup X_{4}}\right)+r\left(\overline{X_{4} \cup X_{5}}\right)-5 r(M) \\
= & r^{*}\left(X_{1} \cup X_{3}\right)+r^{*}\left(X_{1} \cup X_{5}\right)-5 r(M)+12+9+9 \\
& +9+9+2+4+4+5+4 \\
= & r^{*}\left(X_{1} \cup X_{3}\right)+r^{*}\left(X_{1} \cup X_{5}\right)-5 r(M)+67
\end{aligned}
$$

We must have that

$$
\begin{aligned}
& r^{*}\left(X_{1} \cup X_{2}\right)+r^{*}\left(X_{1} \cup X_{3} \cup X_{5}\right) \\
> & r^{*}\left(X_{1} \cup X_{3}\right)+r^{*}\left(X_{1} \cup X_{5}\right)+6
\end{aligned}
$$

in order for this to be a bad family.

Suppose $Z=\varnothing$. Then we have that $7+9>9+8+6$ which is untrue. Now suppose $Z$ is non-empty. Note $X_{1} \cup X_{3} \cup X_{5}$ is equal to the entire ground set, so changing $Z$ has no effect on this term and it is thus still spanning. Now take $r^{*}\left(X_{1} \cup X_{3}\right)=r^{*}\left(V_{2} \cup V_{3} \cup V_{4} \cup V_{5} \cup Z\right)$. As $\overline{X_{1} \cup X_{3}}=V_{1}-Z$ is coindependent for any choice of $Z, X_{1} \cup X_{3}$ is spanning for any choice of $Z$.

Now note that $X_{1} \cup X_{2}=V_{1} \cup V_{2} \cup V_{i} \cup Z$ and $\overline{X_{1} \cup X_{2}}=\left(V_{j} \cup V_{k}\right)-Z$. Note that $r\left(V_{j} \cup V_{k}\right)=4$. If $Z=\varnothing$, then $r\left(\overline{X_{1} \cup X_{2}}\right)=4$. If $Z$ is equal to one element in $V_{j} \cup V_{k}$, the cardinality of $X_{1} \cup X_{2}$ will increase by one but the rank of $\overline{X_{1} \cup X_{2}}$ will be unchanged. This increases $r^{*}\left(X_{1} \cup X_{2}\right)$ by one. Likewise, if $Z$ is equal to two elements of $V_{j} \cup V_{k}$, then $r^{*}\left(X_{1} \cup X_{2}\right)$ increases by two. If $Z$ has cardinality greater than or equal to two, $\left(V_{j} \cup V_{k}\right)-Z$ will be coindependent, making $X_{1} \cup X_{2}$ spanning for all such $Z$. This means the left-hand side of the inequality can increase by at most two.

Finally, $\left|X_{1} \cup X_{5}\right|=\left|V_{1} \cup V_{2} \cup V_{j} \cup Z\right|$ and $\overline{X_{1} \cup X_{5}}=\left(V_{i} \cup V_{k}\right)-Z$. Note that $r\left(V_{i} \cup\right.$ $\left.V_{k}\right)=5$. If $Z$ is equal to one element in $V_{i} \cup V_{k}$, the cardinality of $X_{1} \cup X_{5}$ will increase by one but the rank of $\overline{X_{1} \cup X_{5}}$ will be unchanged. This increases $r^{*}\left(X_{1} \cup\right.$ $\left.X_{5}\right)$ by one. When $Z$ is equal to one or more elements in $V_{i} \cup V_{k},\left(V_{i} \cup V_{k}\right)-Z$ is coindependent. This means that $X_{1} \cup X_{5}$ is spanning for all such $Z$, and increasing $Z$ further can have no effect. This means the right-hand side of the inequality can only increase in value by at most one with a non-empty $Z$. 
The inequality willl not be satisfied for any choice of $Z$.

Subcase 4.1b: $X_{1}=V_{2} \cup Z$ where $Z \subseteq E(M)$

$$
\begin{aligned}
& X_{2}=V_{i} \cup V_{1} \\
& X_{3}=V_{3} \cup V_{4} \cup V_{5} \\
& X_{4}=V_{k} \cup V_{1} \\
& X_{5}=V_{j}
\end{aligned}
$$

Compare this to Subcase 4.1a. $X_{4}$ now contains $V_{1}$ and $X_{5}$ does not, while in Subcase 4.1 a this was the opposite way around. First consider the left-hand side of the inequality. We have that $r^{*}\left(X_{4}\right)+r^{*}\left(X_{5}\right)$ is unchanged, as is $r^{*}\left(X_{2} \cup X_{4} \cup X_{5}\right)$. As $V_{1}$ is contained in $X_{2}, r^{*}\left(X_{2} \cup X_{3} \cup X_{4}\right)$ and $r^{*}\left(X_{2} \cup X_{4} \cup X_{5}\right)$ are also unchanged. The only possible change from Subcase $4.1 \mathrm{a}$ is thus in $r^{*}\left(X_{1} \cup X_{3} \cup X_{5}\right)$. Note that $X_{1} \cup X_{3} \cup X_{5}=V_{2} \cup V_{i} \cup V_{j} \cup V_{k} \cup Z$ and $\overline{X_{1} \cup X_{3} \cup X_{5}}=V_{1}-Z$. As $V_{1}-Z$ is coindependent for any value of $Z, X_{1} \cup X_{3} \cup X_{5}$ is spanning, as in Subcase 4.1a. The left-hand side of the inequality is thus unchanged from Subcase 4.1a.

Now consider the right-hand side of the inequality. We have that $r^{*}\left(X_{2} \cup X_{4}\right)+$ $r^{*}\left(X_{2} \cup X_{5}\right)$ remains the same, as does $r^{*}\left(X_{4} \cup X_{5}\right)$. This leaves $r^{*}\left(X_{1} \cup X_{5}\right)$ and $r^{*}\left(X_{3} \cup X_{4}\right)$. First note that $X_{3} \cup X_{4}=V_{1} \cup V_{3} \cup V_{4} \cup V_{5}$ and $\overline{X_{3} \cup X_{4}}=V_{2}$, which is coindependent, and so $X_{3} \cup X_{4}$ is still spanning. Now take $X_{1} \cup X_{5}=V_{2} \cup V_{j} \cup Z$, where $\overline{X_{1} \cup X_{5}}=\left(V_{1} \cup V_{i} \cup V_{k}\right)-Z$. Suppose $Z=\varnothing$. As $V_{i} \cup V_{k}$ is spanning, $r^{*}\left(X_{1} \cup X_{5}\right)$ will fall by three in comparison to Subcase 4.1a. As $V_{1} \cup V_{i} \cup V_{k}$ is a dependent set of rank 5 , we can remove at most three elements from it without affecting the rank. Thus we can increase $\left|X_{1} \cup X_{5}\right|$ by three without affecting $r^{*}\left(X_{1} \cup X_{5}\right)$, but, after that, any change in $\left|X_{1} \cup X_{5}\right|$ is matched by a decrease in $r\left(\overline{X_{1} \cup X_{5}}\right)$, causing $r^{*}\left(X_{1} \cup X_{5}\right)$ to remain the same.

Thus, in comparison to Subcase 4.1a, the inequality is at worst three lower on the right-hand side. As we showed in that subcase that the left-hand side can increase by at most two with a non-empty choice of $Z$, the inequality cannot be satisfied.

Subcase 4.1c: $X_{1}=V_{2} \cup Z$ where $Z \subseteq E(M)$

$$
\begin{aligned}
& X_{2}=V_{i} \\
& X_{3}=V_{3} \cup V_{4} \cup V_{5} \\
& X_{4}=V_{k} \cup V_{1} \\
& X_{5}=V_{j} \cup V_{1}
\end{aligned}
$$


Compared to subcase 4.1a, $X_{5}$ now contains $V_{1}$ and $X_{2}$ does not.

Consider the left-hand side of the inequality. Any terms which do not involve $X_{2}$ or $X_{5}$ will be unchanged from Subcase 4.1a. Note $\left|X_{5}\right|$ increases by three, while $r\left(\overline{X_{5}}\right)$ remains the same, causing $r^{*}\left(X_{5}\right)$ to increase by three. Also, $r^{*}\left(X_{2} \cup X_{3} \cup X_{4}\right)$ and $r^{*}\left(X_{2} \cup X_{4} \cup X_{5}\right)$ remain the same, as $V_{1} \subset X_{4}$. Finally, take $X_{1} \cup X_{2}=V_{2} \cup V_{i} \cup Z$. We have that $r^{*}\left(V_{2} \cup V_{i}\right)=5$, which is two less than in Subcase 4.1a. As $\overline{X_{1} \cup X_{2}}=$ $\left(V_{1} \cup V_{j} \cup V_{k}\right)-Z$ is a dependent set of rank 5, we can increase $\left|X_{1} \cup X_{2}\right|$ by three without changing $r\left(\overline{X_{1} \cup X_{2}}\right)$. If the cardinality of $Z \subseteq V_{1} \cup V_{j} \cup V_{k}$ is any greater, $\overline{X_{1} \cup X_{2}}$ is coindependent, and so $X_{1} \cup X_{2}$ is spanning for all such $Z$. Thus, in total, the left-hand side increases in value by at most one.

Now take the right-hand side of the inequality. Note that $X_{2} \cup X_{3}$ is still spanning, as $\overline{X_{2} \cup X_{3}}=V_{2}$ is coindependent, and note that $r^{*}\left(X_{2} \cup X_{4}\right)$ is unchanged. Also, $r^{*}\left(X_{2} \cup X_{5}\right)$ and $r^{*}\left(X_{4} \cup X_{5}\right)$ remain the same, as $V_{1} \subset X_{5}$. The right-hand side thus does not change in value.

The inequality does not hold, and we thus have no bad family for any choice of $Z$.

Subcase 4.2a: $X_{1}=V_{2} \cup Z$ where $Z \subseteq E(M)$

$$
\begin{aligned}
& X_{2}=V_{i} \cup V_{1} \\
& X_{3}=V_{3} \cup V_{4} \cup V_{5} \\
& X_{4}=V_{k} \cup V_{1} \\
& X_{5}=V_{j} \cup\{a\} \text { where } a \in V_{1}
\end{aligned}
$$

Note that $\overline{X_{2} \cup X_{3} \cup X_{4}}=\overline{X_{2} \cup X_{4} \cup X_{5}}=\overline{V_{1} \cup V_{i} \cup V_{j} \cup V_{k}}=V_{2}$.

$$
\begin{aligned}
\text { LHS }= & r^{*}\left(X_{3}\right)+r^{*}\left(X_{4}\right)+r^{*}\left(X_{5}\right)+r^{*}\left(X_{1} \cup X_{2}\right)+r^{*}\left(X_{1} \cup X_{3} \cup X_{5}\right) \\
& +r^{*}\left(X_{2} \cup X_{3} \cup X_{4}\right)+r^{*}\left(X_{2} \cup X_{4} \cup X_{5}\right) \\
=r^{*} & \left(X_{1} \cup X_{2}\right)+r^{*}\left(X_{1} \cup X_{3} \cup X_{5}\right)+\left|X_{3}\right|+\left|X_{4}\right|+\left|X_{5}\right| \\
& \quad+\left|X_{2} \cup X_{3} \cup X_{4}\right|+\left|X_{2} \cup X_{4} \cup X_{5}\right|+r\left(\overline{X_{3}}\right)+r\left(\overline{X_{4}}\right) \\
& +r\left(\overline{X_{5}}\right)+r\left(\overline{X_{2} \cup X_{3} \cup X_{4}}\right)+r\left(\overline{X_{2} \cup X_{4} \cup X_{5}}\right)-5 r(M) \\
= & r^{*}\left(X_{1} \cup X_{2}\right)+r^{*}\left(X_{1} \cup X_{3} \cup X_{5}\right)-5 r(M)+9+6+4 \\
& \quad+12+12+5+5+5+2+2 \\
= & r^{*}\left(X_{1} \cup X_{2}\right)+r^{*}\left(X_{1} \cup X_{3} \cup X_{5}\right)-5 r(M)+62
\end{aligned}
$$




$$
\begin{aligned}
& R H S=r^{*}\left(X_{1} \cup X_{3}\right)+r^{*}\left(X_{1} \cup X_{5}\right)+r^{*}\left(X_{2} \cup X_{3}\right)+r^{*}\left(X_{2} \cup X_{4}\right) \\
&+r^{*}\left(X_{2} \cup X_{5}\right)+r^{*}\left(X_{3} \cup X_{4}\right)+r^{*}\left(X_{4} \cup X_{5}\right) \\
&=r^{*}\left(X_{1} \cup X_{3}\right)+r^{*}\left(X_{1} \cup X_{5}\right)+\left|X_{2} \cup X_{3}\right|+\left|X_{2} \cup X_{4}\right|+\left|X_{2} \cup X_{5}\right| \\
&+\left|X_{3} \cup X_{4}\right|+\left|X_{4} \cup X_{5}\right|+r\left(\overline{X_{2} \cup X_{3}}\right)+r\left(\overline{X_{2} \cup X_{4}}\right) \\
&+r\left(\overline{X_{2} \cup X_{5}}\right)+r\left(\overline{X_{3} \cup X_{4}}\right)+r\left(\overline{X_{4} \cup X_{5}}\right)-5 r(M) \\
&=r^{*}\left(X_{1} \cup X_{3}\right)+r^{*}\left(X_{1} \cup X_{5}\right)-5 r(M)+12+9+9 \\
& \quad+12+9+2+4+4+2+4 \\
&=r^{*}\left(X_{1} \cup X_{3}\right)+r^{*}\left(X_{1} \cup X_{5}\right)-5 r(M)+67
\end{aligned}
$$

We must have that

$$
\begin{aligned}
& r^{*}\left(X_{1} \cup X_{2}\right)+r^{*}\left(X_{1} \cup X_{3} \cup X_{5}\right) \\
> & r^{*}\left(X_{1} \cup X_{3}\right)+r^{*}\left(X_{1} \cup X_{5}\right)+5
\end{aligned}
$$

in order for this to be a bad family.

Suppose $Z=\varnothing$. We have that $7+9>9+6+5$ which is untrue. Now suppose $Z \neq \varnothing$. We have shown in Subcase 4.1a that $r^{*}\left(X_{1} \cup X_{3}\right)$ cannot change, and that $r^{*}\left(X_{1} \cup X_{2}\right)$ can increase by at most two. The sets $X_{1}, X_{2}$, and $X_{3}$ are the same in the current subcase and hence the same facts apply. Note that $X_{1} \cup X_{3} \cup X_{5}$ is equal to the entire ground set, and thus changing $Z$ will have no effect on this term, as in Subcase 4.1a. Let $V_{1}=\{a, b, c\}$. Finally, note that $X_{1} \cup X_{5}=\{a\} \cup V_{2} \cup V_{j} \cup Z$ and $\overline{X_{1} \cup X_{5}}=\left(\{b, c\} \cup V_{i} \cup V_{k}\right)-Z$. As $\{b, c\} \cup V_{i} \cup V_{k}$ is a dependent set of rank 5 , we can remove at most three elements from it without affecting the rank. Thus we can increase $\left|X_{1} \cup X_{5}\right|$ by three without affecting $r^{*}\left(X_{1} \cup X_{5}\right)$, but, after that, $\overline{X_{1} \cup X_{5}}$ is coindependent, causing $r^{*}\left(X_{1} \cup X_{5}\right)$ to be spanning for all such $Z$. Thus the right-hand side of the inequality can increase by at most three when we make $Z$ non-empty. The left-hand side can increase by at most two, and so the inequality cannot be satisfied.

Subcase 4.2b: $X_{1}=V_{2} \cup Z$ where $Z \subseteq E(M)$

$$
\begin{aligned}
& X_{2}=V_{i} \cup\{a\} \text { where } a \in V_{1} \\
& X_{3}=V_{3} \cup V_{4} \cup V_{5} \\
& X_{4}=V_{k} \cup V_{1} \\
& X_{5}=V_{j} \cup V_{1}
\end{aligned}
$$


Compared to Subcase 4.2a, $X_{2}$ contains two less elements of $V_{1}$ while $X_{5}$ contains two more.

Consider the left-hand side of the inequality. Any terms not involving $X_{2}$ or $X_{5}$ are unchanged from Subcase 4.2a. As $\left|X_{5}\right|$ increases by two in comparison to Subcase 4.2a, while $r\left(\overline{X_{5}}\right)$ remains the same, we have that $r^{*}\left(X_{5}\right)$ to increase by two. As $V_{1} \subset X_{4}, r^{*}\left(X_{2} \cup X_{3} \cup X_{4}\right)$ and $r^{*}\left(X_{2} \cup X_{4} \cup X_{5}\right)$ remain the same. Note that $X_{1} \cup X_{3} \cup X_{5}$ is equal to the entire ground set and thus must be spanning, as in Subcase 4.2a. Now note that $X_{1} \cup X_{2}=\{a\} \cup V_{2} \cup V_{i} \cup Z$ and $\overline{X_{1} \cup X_{2}}=$ $\left(\{b, c\} \cup V_{i} \cup V_{k}\right)-Z$. As $\{b, c\} \cup V_{i} \cup V_{k}$ is a dependent set of rank 5, we can remove at most three elements from it without affecting the rank. Thus we can increase $\left|X_{1} \cup X_{2}\right|$ by three without affecting $r^{*}\left(X_{1} \cup X_{2}\right)$, but, after that, $\overline{X_{1} \cup X_{2}}$ is coindependent, causing $r^{*}\left(X_{1} \cup X_{2}\right)$ to be spanning for all such $Z$. The left-hand side can thus increase by at most five in comparison to Subcase 4.2a.

Take the right-hand side of the inequality. Note $r^{*}\left(X_{2} \cup X_{5}\right)$ is unchanged. As $V_{1} \subset X_{4}, r^{*}\left(X_{2} \cup X_{4}\right)$ and $r^{*}\left(X_{4} \cup X_{5}\right)$ are also unchanged. We have that $\left|X_{2} \cup X_{3}\right|$ decreases by two, but $r\left(\overline{X_{2} \cup X_{3}}\right)$ increases by two, meaning that $r^{*}\left(X_{2} \cup X_{3}\right)$ is unchanged. Finally, take $X_{1} \cup X_{5}=V_{1} \cup V_{2} \cup V_{j} \cup Z$, where $\overline{X_{1} \cup X_{5}}=\left(V_{i} \cup V_{k}\right)-Z$. Suppose that, to begin with, $Z=\varnothing$. In Subcase 4.2a, $r^{*}\left(X_{1} \cup X_{5}\right)=6$. As $\overline{X_{1} \cup X_{5}}$ is still spanning in the current subcase, $r^{*}\left(X_{1} \cup X_{5}\right)$ increases by two due to the increase in $\left|X_{1} \cup X_{2}\right|$. Now suppose $Z$ is non-empty. As $V_{i} \cup V_{k}$ has rank 5, we can increase $\left|X_{1} \cup X_{5}\right|$ by one without decreasing $r\left(\overline{X_{1} \cup X_{5}}\right)$. For any $Z \subseteq V_{i} \cup V_{k}$ with a cardinality greater than or equal to one, $\overline{X_{1} \cup X_{5}}$ is coindependent. This means $X_{1} \cup X_{5}$ is spanning for all such $Z$, and so $r^{*}\left(X_{1} \cup X_{5}\right)$ can increase by at most one with a non-empty $Z$. Thus, in total, the right-hand side of the inequality can increase by at most three. We cannot have a bad family for any choice of $Z$.

Subcase 4.2c: $X_{1}=V_{2} \cup Z$ where $Z \subseteq E(M)$

$$
\begin{aligned}
& X_{2}=V_{i} \cup V_{1} \\
& X_{3}=V_{3} \cup V_{4} \cup V_{5} \\
& X_{4}=V_{k} \cup\{a\} \text { where } a \in V_{1} \\
& X_{5}=V_{j} \cup V_{1}
\end{aligned}
$$

Compared to Subcase 4.2a, $X_{4}$ contains two less elements of $V_{1}$ while $X_{5}$ contains two more.

On the left-hand side of the inequality, there is no change in value compared to 
Subcase 4.2a. Note that $r^{*}\left(X_{4}\right)+r^{*}\left(X_{5}\right)$ remains the same, as does $r^{*}\left(X_{2} \cup X_{4} \cup\right.$ $\left.X_{5}\right)$. In Subcase $4.2 \mathrm{a}, X_{1} \cup X_{3} \cup X_{5}$ was spanning and the same is true after adding additional elements to it. As $V_{1} \subset X_{2}, r^{*}\left(X_{2} \cup X_{4} \cup X_{4}\right)$ is also unchanged.

Now take the right-hand side of the inequality. We have that $r^{*}\left(X_{4} \cup X_{5}\right)$ is unchanged, as are $r^{*}\left(X_{2} \cup X_{4}\right)$ and $r^{*}\left(X_{2} \cup X_{5}\right)$ since $V_{1} \subset X_{2}$. As in Subcase $4.2 \mathrm{~b}$, $r^{*}\left(X_{1} \cup X_{5}\right)$ can increase by at most three. Finally, take $X_{3} \cup X_{4}=\{a\} \cup V_{3} \cup V_{4} \cup V_{5}$. This set is spanning, as in Subcase 4.2a.

We have that, in comparison to Subcase 4.2a, the left-hand side remains the same while the right-hand side increases by at most three. We still have no bad family, for any choice of $Z$.

Subcase 4.3: $X_{1}=V_{2} \cup Z$ where $Z \subseteq E(M)$

$$
\begin{aligned}
& X_{2}=V_{i} \cup V_{1} \\
& X_{3}=V_{3} \cup V_{4} \cup V_{5} \\
& X_{4}=V_{k} \cup V_{1} \\
& X_{5}=V_{j} \cup V_{1}
\end{aligned}
$$

Note that $\overline{X_{2} \cup X_{3} \cup X_{4}}=\overline{X_{2} \cup X_{4} \cup X_{5}}=\overline{V_{1} \cup V_{i} \cup V_{j} \cup V_{k}}=V_{2}$.

$$
\begin{aligned}
& \text { LHS }=r^{*}\left(X_{3}\right)+r^{*}\left(X_{4}\right)+r^{*}\left(X_{5}\right)+r^{*}\left(X_{1} \cup X_{2}\right)+r^{*}\left(X_{1} \cup X_{3} \cup X_{5}\right) \\
& +r^{*}\left(X_{2} \cup X_{3} \cup X_{4}\right)+r^{*}\left(X_{2} \cup X_{4} \cup X_{5}\right) \\
& =r^{*}\left(X_{1} \cup X_{2}\right)+r^{*}\left(X_{1} \cup X_{3} \cup X_{5}\right)+\left|X_{3}\right|+\left|X_{4}\right|+\left|X_{5}\right| \\
& +\left|X_{2} \cup X_{3} \cup X_{4}\right|+\left|X_{2} \cup X_{4} \cup X_{5}\right|+r\left(\overline{X_{3}}\right)+r\left(\overline{X_{4}}\right) \\
& +r\left(\overline{X_{5}}\right)+r\left(\overline{X_{2} \cup X_{3} \cup X_{4}}\right)+r\left(\overline{X_{2} \cup X_{4} \cup X_{5}}\right)-5 r(M) \\
& =r^{*}\left(X_{1} \cup X_{2}\right)+r^{*}\left(X_{1} \cup X_{3} \cup X_{5}\right)-5 r(M)+9+6+6 \\
& +12+12+5+5+5+2+2 \\
& =r^{*}\left(X_{1} \cup X_{2}\right)+r^{*}\left(X_{1} \cup X_{3} \cup X_{5}\right)-5 r(M)+64
\end{aligned}
$$




$$
\begin{aligned}
& R H S=r^{*}\left(X_{1} \cup X_{3}\right)+r^{*}\left(X_{1} \cup X_{5}\right)+r^{*}\left(X_{2} \cup X_{3}\right)+r^{*}\left(X_{2} \cup X_{4}\right) \\
&+r^{*}\left(X_{2} \cup X_{5}\right)+r^{*}\left(X_{3} \cup X_{4}\right)+r^{*}\left(X_{4} \cup X_{5}\right) \\
&=r^{*}\left(X_{1} \cup X_{3}\right)+r^{*}\left(X_{1} \cup X_{5}\right)+\left|X_{2} \cup X_{3}\right|+\left|X_{2} \cup X_{4}\right|+\left|X_{2} \cup X_{5}\right| \\
&+\left|X_{3} \cup X_{4}\right|+\left|X_{4} \cup X_{5}\right|+r\left(\overline{X_{2} \cup X_{3}}\right)+r\left(\overline{X_{2} \cup X_{4}}\right) \\
&+r\left(\overline{X_{2} \cup X_{5}}\right)+r\left(\overline{X_{3} \cup X_{4}}\right)+r\left(\overline{X_{4} \cup X_{5}}\right)-5 r(M) \\
&=r^{*}\left(X_{1} \cup X_{3}\right)+r^{*}\left(X_{1} \cup X_{5}\right)-5 r(M)+12+12+9 \\
& \quad+12+9+2+4+4+2+4 \\
&=r^{*}\left(X_{1} \cup X_{3}\right)+r^{*}\left(X_{1} \cup X_{5}\right)-5 r(M)+70
\end{aligned}
$$

We must have that

$$
\begin{aligned}
& r^{*}\left(X_{1} \cup X_{2}\right)+r^{*}\left(X_{1} \cup X_{3} \cup X_{5}\right) \\
> & r^{*}\left(X_{1} \cup X_{3}\right)+r^{*}\left(X_{1} \cup X_{5}\right)+6
\end{aligned}
$$

in order for this to be a bad family.

Suppose $Z=\varnothing$. Then we have that $7+9>9+8+6$ which is untrue. We have shown in Subcase 4.1a that $r^{*}\left(X_{1} \cup X_{3}\right)$ cannot change when $Z$ is non-empty, and that $r^{*}\left(X_{1} \cup X_{2}\right)$ can increase by at most two. The sets $X_{1}, X_{2}$, and $X_{3}$ are the same in the current subcase and hence the same facts apply. Note that $X_{1} \cup X_{3} \cup X_{5}$ is equal to the entire ground set, and thus changing $Z$ will have no effect on this term. Finally, $X_{1} \cup X_{5}=V_{1} \cup V_{2} \cup V_{j} \cup Z$ and $\overline{X_{1} \cup X_{5}}=\left(V_{i} \cup V_{k}\right)-Z$. As $r\left(V_{i} \cup V_{k}\right)=5$, we can remove one element from it without decreasing the rank. This increases $\left|X_{1} \cup X_{5}\right|$ by one and therefore $r^{*}\left(X_{1} \cup X_{5}\right)$. For any $Z \subseteq V_{i} \cup V_{k}$ with cardinality one or higher, $\overline{X_{1} \cup X_{5}}$ is coindependent, so $X_{1} \cup X_{5}$ is spanning for all such $Z$. We thus have that the left-hand side can increase by at most two, while the right-hand side can increase by at most one, giving us no possible bad family. 


\section{Chapter 6}

\section{A Complexity Theorem}

As yet no method of testing whether a matroid satisfies a Kinser equality has presented itself other than brute force. This leads to the question of whether it is possible to do this in polynomial time. Given the increasing number of terms in each inequality and the lack of bounds on a matroids possible ground set, this is an important question in terms of the results it is feasible to get - in particular, whether it would be feasible to construct a matroid similar to that used in Theorem 5.1 and test whether it satisfies inequality $n$ for $n \geq 5$, in order to show that the higher Kinser classes are not dual closed. We give a proof that it would in fact be impossible to test these in polynomial time.

An oracle machine consists of a Turing machine with a black box attached, which is referred to as the oracle. Given some question about a particular matroid, inputs are fed to the oracle, which then gives an output answering the question. The time the machine takes to produce an output is given as a function of the number of inputs necessary to answer the question. We wish to know the time an oracle machine would take to answer whether a matroid satisfies Kinser inequality $n$.

Definition 6.1. Let $r \geq 4$ and take two distinct $r$-element sets $A=\left\{a_{1}, \ldots, a_{r}\right\}$ and $B=\left\{b_{1}, \ldots, b_{r}\right\}$. We define the circuit-hyperplanes of the rank-r binary spike, denoted by $Z_{r}$, on ground set $E=A \cup B$ by its set of circuits. First, define the set of circuit-hyperplanes to be the subsets $\left\{z_{1}, \ldots, z_{r}\right\}$, where $z_{i} \in\left\{a_{i}, b_{i}\right\}$, such that $\left|\left\{z_{1}, \ldots, z_{r}\right\} \cap\left\{b_{1}, \ldots, b_{r}\right\}\right|$ is even. The non-spanning circuits of $Z_{r}$ consist of the circuit-hyperplanes as defined above and subsets of $E$ of the form $\left\{a_{i}, b_{i}, a_{k}, b_{k}\right\}$. 
$Z_{r}$ can be represented by the following matrix:

$$
\left[I_{r} \mid[1]_{r \times r}-I_{r}\right]
$$

Lemma 6.2. Take an arbitrary rank $r$ binary spike where $r$ is even. If we relax any circuit-hyperplane other than A, the resulting matroid violates the Ingleton condition.

Proof. Take a binary spike $Z_{r}$. Take one of the circuit-hyperplanes of $Z_{r}$ and call it $Z$, where $Z$ is chosen so that $Z \cap A$ and $Z \cap B$ are non-empty. Define $I \subseteq\{1, \ldots, r\}$ such that $i \in I$ if and only if $a_{i} \in Z$, and define $J \subseteq\{1, \ldots, r\}$ such that $j \in J$ if and only if $b_{i} \in Z$. Now let $X_{1}=\left\{a_{i} \mid i \in I\right\}, X_{2}=\left\{b_{j} \mid j \in J\right\}, X_{3}=\left\{b_{i} \mid i \in I\right\}$, and $X_{4}=\left\{a_{j} \mid j \in J\right\}$. In other words, $X_{1}$ and $X_{2}$ consist of the elements in the circuit-hyperplane $Z$ contained in $A$ and $B$ respectively, while $X_{3}$ and $X_{4}$ consist of all the remaining elements in $B$ and $A$. Note that $X_{2}$ contains an even number of elements from $B$ and that $\left|X_{1} \cup X_{2}\right|=r$, making it a circuit-hyperplane. Relax $X_{1} \cup X_{2}$ to get the matroid $Z_{r}^{-}$and evaluate the Ingleton condition:

$$
\begin{aligned}
& r\left(X_{3}\right)+r\left(X_{4}\right)+r\left(X_{1} \cup X_{2}\right)+r\left(X_{1} \cup X_{3} \cup X_{4}\right)+r\left(X_{2} \cup X_{3} \cup X_{4}\right) \\
\leq & r\left(X_{1} \cup X_{3}\right)+r\left(X_{1} \cup X_{4}\right)+r\left(X_{2} \cup X_{3}\right)+r\left(X_{2} \cup X_{4}\right)+r\left(X_{3} \cup X_{4}\right)
\end{aligned}
$$

The set of non-spanning circuits of $Z_{r}$ consists of the circuit-hyperplanes as defined above and subsets of $E$ of the form $\left\{a_{i}, b_{i}, a_{k}, b_{k}\right\} . X_{3}$ and $X_{4}$ do not fit into this category and are thus independent. The ground set of $Z_{r}^{-}$has size $2 r$ and $Z$ has size $r$, so $X_{3}$ and $X_{4}$ have ranks which sum to $r$. A leg is a subset of the ground set of $Z_{r}$ of the form $\left\{a_{k}, b_{k}\right\}$ for some $k$. A proper subset of the legs has rank one greater than the number of legs. $X_{1} \cup X_{3}=\left\{a_{i} \cup b_{i} \mid i \in I\right\}$ and $X_{2} \cup X_{4}=\left\{a_{j} \cup b_{j} \mid j \in J\right\}$ are both collections of legs, the former having $\left|X_{1}\right|=\left|X_{3}\right|$ legs and the latter having $\left|X_{2}\right|=\left|X_{4}\right|$ legs. Thus $r\left(X_{1} \cup X_{3}\right)=\left|X_{1}\right|+1$ and $r\left(X_{2} \cup X_{4}\right)=\left|X_{2}\right|+1$. As $Z$ is a circuit-hyperplane, $\left|X_{2}\right|$ is even by definition. This means $X_{2} \cup X_{3}$ is a circuit-hyperplane. Recall that $X_{2}$ and $X_{3}$ partition $B$. As $r$ is even, $\left|X_{3}\right|=r-\left|X_{2}\right|$ must be even as well. Thus $X_{3} \cup X_{4}$ is a circuit-hyperplane, as is $X_{1} \cup X_{4}=A$. Now consider $X_{1} \cup X_{3} \cup X_{4}$. This set properly contains the circuit-hyperplane $X_{3} \cup X_{4}$, and all the sets $X_{i}$ are non-empty. Thus $X_{1} \cup X_{3} \cup X_{4}$ is spanning. The set $X_{2} \cup X_{3} \cup X_{4}$ also properly contains a circuit-hyperplane, and so is also spanning. Using these calculations we can now evaluate the inequality. 


$$
4 r \leq\left(\left|X_{1}\right|+1\right)+(r-1)+(r-1)+\left(\left|X_{2}\right|+1\right)+(r-1)
$$

Since $\left|X_{1}\right|+\left|X_{2}\right|=r$, this simplifies to $4 r \leq 4 r-1$ which is untrue. Therefore $X_{1}, \ldots, X_{4}$ form a bad family.

Theorem 6.3. Let $n \geq 4$. There does not exist a polynomial time oracle machine testing Kinser inequality $n$ or its dual.

Proof. As proved above, each binary spike $Z_{r}$ of even rank is representable, therefore satisfies the inequality, while its relaxation $Z_{r}^{-}$does not. This means that in order to test whether a matroid satisfies Kinser inequality $n$ or its dual, the oracle machine must distinguish between each $Z_{r}$ and $Z_{r}^{-}$. Recall $Z_{r}^{-}$can be constructed by relaxing any circuit-hyperplane, which consists of an $r$ element subset $\left\{z_{1}, \ldots, z_{r}\right\}$ of the ground set $A \cup B$ where $z_{i} \in\left\{a_{i}, b_{i}\right\}$ and $\left|\left\{z_{1}, \ldots, z_{r}\right\} \cap\left\{b_{1}, \ldots, b_{r}\right\}\right|$ is even. Suppose the oracle did not check the rank of the relaxed circuit-hyperplane. This would mean it yields the same result as before the circuit-hyperplane was relaxed, as that is the only subset which changes in rank. Thus the oracle must check the rank of each possible circuit-hyperplane. There are $2^{r} r$-element sets using one element from each leg, and half of these contain an even number of elements from $\left\{b_{1}, \ldots, b_{r}\right\}$. The algorithm hence takes at least $2^{r-1}=2^{\frac{E}{2}-1}$ checks, and therefore is exponential in the size of the ground set. As the class of spikes is dual-closed, testing whether the dual of a matroid satisfies Kinser inequality $n$ is also exponential in the size of the ground set. 


\section{Chapter 7}

\section{Excluded minors}

The following theorem was proved by Mayhew, Newman, and Whittle in 2008 [6], settling a conjecture by Geelen [2].

Theorem 7.1. For any infinite field $\mathbb{K}$ and any matroid $N$ representable over $\mathbb{K}$, there is an excluded minor for $\mathbb{K}$-representability that has $N$ as a minor.

The proof of Theorem 7.1 constructed an excluded minor which contained $N$ and which was not contained in $\mathcal{K}_{4}$, and thus was not contained inside any Kinser class. In Theorem 7.3 we give a strengthening of this result, which states that the excluded minors can actually be contained inside any layer of the hierarchy.

Lemma 7.2. Let $r \geq 3$ be an integer. Let $P$ be the projective geometry $P G(r-$ $1, \mathbb{K})$, where $\mathbb{K}$ is an infinite field, and let $S_{1}, \ldots, S_{t}$ be a finite collection of proper subspaces of $P$. If $S$ is a subspace of $P$ that is not contained in any of $S_{1}, \ldots, S_{t}$, then $S$ is not contained in $S_{1} \cup \ldots \cup S_{t}$.

This is Proposition 4.2 of [5] and we will make frequent reference to it throughout this chapter. Whenever we add points freely to a subspace, it is justified by this result.

Theorem 7.3. Let $n \geq 5$ be an integer. Let $\mathbb{K}$ be a infinite field and let $M$ be a $\mathbb{K}$-representable matroid. Then $M$ is contained in an excluded minor for $\mathcal{K}_{n+1}$ which is in $\mathcal{K}_{n}$.

Proof. As we can add coloops as desired, we can assume $M$ has rank $r$ where $r \geq$ $n$. By [6, Lemma 2.2], we can assume that $M$ is partitioned into two independent 
hyperplanes. Call these $H_{0}$ and $H_{n-1}$. Let $\mathbb{K}$ be an infinite field. Imbed $M$ in the projective geometry $P=P G(r, \mathbb{K})$, so that the elements in the ground set of $M$ are identified with points in $P$. Note that this geometry has rank $r+1$, so $M$ spans a hyperplane of $P$. If $X$ is any set of points in $P$, let $\langle X\rangle$ denote the closure of $X$ in $P$. We will now extend $M$ to get $N$, an excluded minor for $\mathcal{K}_{n+1}$. First we will choose points which will not be added to the ground set of $M$, but will enable us to freely place points within $M$.

Begin by arbitrarily choosing $x_{0}$ in $P-\langle E(M)\rangle$. Next freely place $x_{n-1}$ with respect to $\left\langle H_{0}\right\rangle$-i.e., choose $x_{n-1}$ in $\left\langle H_{0}\right\rangle$ so that $x_{n-1}$ is not spanned by any subset of $E(M) \cup\left\{x_{0}\right\}$ that doesn't span $H_{0}$. We are able to do this using Lemma7.2.

Choose $x_{1}$ in $\left\langle H_{n-1}\right\rangle$ so that it is not spanned by any subset of $E(M) \cup\left\{x_{0}, x_{n-1}\right\}$ that doesn't span $H_{n-1}$. Now choose $x_{2}$ in $\left\langle H_{0}\right\rangle \cap\left\langle H_{n-1}\right\rangle$ so that it is not spanned by any subset of $E(M) \cup\left\{x_{0}, x_{1}, x_{n-1}\right\}$ unless that subset spans $\left\langle H_{0}\right\rangle \cap\left\langle H_{n-1}\right\rangle$. Choose $x_{3}$ in $\left\langle H_{0}\right\rangle \cap\left\langle H_{n-1}\right\rangle$ so that it is not spanned by any subset of $E(M) \cup$ $\left\{x_{0}, x_{1}, x_{2}, x_{n-1}\right\}$ unless that subset spans $\left\langle H_{0}\right\rangle \cap\left\langle H_{n-1}\right\rangle$. Continue in this way until $x_{0}, x_{1}, \ldots, x_{n-1}$ have been chosen. Now choose $r-n+1$ points in the same space, $\left\langle H_{0}\right\rangle \cap\left\langle H_{n-1}\right\rangle$ using the same technique. Call this set of points $X$, and note that $X \cup\left\{x_{2}, \ldots, x_{n-2}\right\}$ is an independent set that spans $\left\langle H_{0}\right\rangle \cap\left\langle H_{n-1}\right\rangle$.

The points chosen so far, $X \cup\left\{x_{0}, \ldots, x_{n-1}\right\}$, will act as guides for adding points to the ground set of $N$. Add a point $e_{1}$ to $\left\langle\left(X \cup\left\{x_{0}, \ldots, x_{n-1}\right\}\right)-\left\{x_{1}, x_{2}\right\}\right\rangle$ so that it is not spanned by any subset of $E(M) \cup X \cup\left\{x_{0}, \ldots, x_{n-1}\right\}$ unless that subset spans $\left(X \cup\left\{x_{0}, \ldots, x_{n-1}\right\}\right)-\left\{x_{1}, x_{2}\right\}$. Now add another point to the same space so that it is not spanned by any subset of $E(M) \cup X \cup\left\{x_{0}, \ldots, x_{n-1}, e_{1}\right\}$ unless that subset spans $\left(X \cup\left\{x_{0}, \ldots, x_{n-1}\right\}\right)-\left\{x_{1}, x_{2}\right\}$. Continue in this way until $r-1$ points have been added to the space. Call this set of $r-1$ points $H_{1}$. Follow this same method to create $r-1$ points to form the set $H_{2}$, this time adding the points to the space $\left\langle\left(X \cup\left\{x_{0}, \ldots, x_{n-1}\right\}\right)-\left\{x_{2}, x_{3}\right\}\right\rangle$. In this way we create $H_{1}, \ldots, H_{n-2}$; that is, for $i \in\{1, \ldots, n-2\}$, create $H_{i}$ by freely placing $r-1$ points in the space $\left\langle\left(X \cup\left\{x_{0}, \ldots, x_{n-1}\right\}\right)-\left\{x_{i}, x_{i+1}\right\}\right\rangle$. Note that the points of $H_{0}$ are in $\left\langle\left(X \cup\left\{x_{0}, \ldots, x_{n-1}\right\}\right)-\left\{x_{0}, x_{1}\right\}\right\rangle$ and the points of $H_{n-1}$ are in $\left\langle\left(X \cup\left\{x_{0}, \ldots, x_{n-1}\right\}\right)-\left\{x_{n-1}, x_{0}\right\}\right\rangle$.

Finally, add a point $p$ freely to $\langle X\rangle$, then add another point $p^{\prime}$ freely to $P$. Freely place a point $e$ on the line spanned by $p$ and $p^{\prime},\left\langle\left\{p, p^{\prime}\right\}\right\rangle$, then do the same with another point $f$. Let $N$ be the matroid consisting of the points $H_{0} \cup \ldots H_{n-1} \cup$ 
$\{e, f\}$.

Lemma 7.4. $N$ is $\mathbb{K}$-representable.

This lemma is true by construction.

Lemma 7.5. $H_{i} \cup\{e, f\}$ is a circuit-hyperplane of $N$ for every $i \in\{0, \ldots, n-1\}$.

Proof. Note $H_{i} \cup\{e, f\}$ has $r+1$ points, and by construction is contained in $\left\langle\left(X \cup\left\{p^{\prime}, x_{0}, \ldots, x_{n-1}\right\}\right)-\left\{x_{i}, x_{i+1}\right\}\right\rangle$. This is a rank $r$ space and so $H_{i} \cup\{e, f\}$ must be dependent. Suppose $H_{i}$ is dependent for some $i$. Then at some point in constructing $N$, we would have added a point $g$ to already chosen elements of $H_{i}$ so that the point was contained in $\operatorname{cl}\left(H_{i}-g\right)$; that is, contained in $\left\langle\left(X \cup\left\{x_{0}, \ldots, x_{n-1}\right\}\right)-\left\{x_{i}, x_{i+1}\right\}\right\rangle$. This contradicts every point of $H_{i}$ being freely placed in the space $\left\langle\left(X \cup\left\{x_{o}, \ldots, x_{n-1}\right\}\right)-\left\{x_{i}, x_{i+1}\right\}\right\rangle$. Thus $H_{i}$ is independent. Now suppose $H_{i} \cup\{e\}$ is dependent. Then $e \in \operatorname{cl}\left(H_{i}\right)$. That is, $e \in\left\langle\left(X \cup\left\{x_{0}, \ldots, x_{n-1}\right\}-\left\{x_{i}, x_{i+1}\right\}\right\rangle\right.$. This contradicts $e$ being a point on the line spanned by $p$ and $p^{\prime}$. Likewise, $H_{i} \cup\{f\}$ is also independent. We have shown that every subset of $H_{i} \cup\{e, f\}$ is independent, meaning that $H_{i} \cup\{e, f\}$ must be a circuit.

Now suppose $H_{i} \cup\{e, f\}$ is not a flat. Then there must be some element $g \in E(N)-\left(H_{i} \cup\{e, f\}\right)$ such that $r\left(H_{i} \cup\{e, f, g\}\right)=r\left(H_{i} \cup\{e, f\}\right)-$ that is, $g \in \operatorname{cl}\left(H_{i} \cup\{e, f\}\right)$. This implies $g \in \operatorname{cl}\left(H_{i} \cup\{e\}\right)$. Let $g \in H_{j}$ for some $j$. Assume $g \in \operatorname{cl}\left(H_{i}\right)$. Then we have that $g \in\left\langle\left(X \cup\left\{x_{0}, \ldots, x_{n-1}\right\}\right)-\left\{x_{i}, i+1\right\}\right\rangle$. This is a contradiction, as $\left(X \cup\left\{x_{0}, \ldots, x_{n-1}\right\}\right)-\left\{x_{i}, x_{i+1}\right\}$ does not span $\left(X \cup\left\{x_{0}, \ldots, x_{n-1}\right\}\right)-$ $\left\{x_{j}, x_{j+1}\right\}$.

Now suppose $g \notin \operatorname{cl}\left(H_{i}\right)$. By the third closure axiom, $e \in \operatorname{cl}\left(H_{i} \cup\{g\}\right)$. Recall that $p \in \operatorname{cl}\left(H_{i}\right)$. As $p$ and $e$ form a line, we must have that $\langle\{p, e\}\rangle \subseteq \operatorname{cl}\left(H_{i} \cup\{g\}\right)$. Thus $p^{\prime} \in \operatorname{cl}\left(H_{i} \cup g\right)$. As $p^{\prime}$ was added freely to the projective geometry, the only way this is possible is if $H_{i} \cup g$ is spanning, which is a contradiction.

Lemma 7.6. Relaxing $H_{0} \cup\{e, f\}$ in $N$ produces a matroid not in $\mathcal{K}_{n+1}$.

Proof. We will show that $\left(X_{1}, X_{2}, \ldots, X_{n+1}\right)=\left(H_{0},\{e, f\}, H_{1}, \ldots, H_{n-1}\right)$ violates 
inequality $n+1$, that is,

$$
\begin{array}{r}
\sum_{i=3}^{n+1} r\left(X_{i}\right)+r\left(X_{1} \cup X_{2}\right)+r\left(X_{1} \cup X_{3} \cup X_{n+1}\right)+\sum_{i=4}^{n+1} r\left(X_{2} \cup X_{i-1} \cup X_{i}\right) \\
>r\left(X_{1} \cup X_{3}\right)+r\left(X_{1} \cup X_{n+1}\right)+\sum_{i=3}^{n+1} r\left(X_{2} \cup X_{i}\right)+\sum_{i=4}^{n+1} r\left(X_{i-1} \cup X_{i}\right)
\end{array}
$$

Recall that $X_{i}$ is independent by construction, as proved in Lemma 7.5, with rank $r-1$. Recall $X_{i} \cup X_{2}$ is a circuit-hyperplane for all $i$ as also proved in Lemma 7.5. Note that $X_{i} \subseteq\left\langle X \cup\left\{x_{0}, \ldots, x_{n-1}\right\}-\left\{x_{i}, x_{i+1}\right\}\right\rangle$ for all $i \neq 2$, and that the points were chosen so as to make it an independent set of rank $r-1$. Take two consecutive sets $X_{i}$ and $X_{j}$, where $i, j \neq 2$.

$$
\begin{aligned}
& r\left(X_{i} \cup X_{j}\right) \\
& \leq \quad r\left(\left\langle X \cup\left\{x_{0}, \ldots, x_{n-1}\right\}-\left\{x_{i}, x_{i+1}\right\}\right\rangle \cup\left\langle X \cup\left\{x_{0}, \ldots, x_{n-1}\right\}-\left\{x_{i+1}, x_{j}\right\}\right\rangle\right) \\
&= r\left(\left\langle X \cup\left\{x_{0}, \ldots, x_{n-1}\right\}-\left\{x_{i}, x_{i+1}\right\}\right\rangle\right)+r\left(\left\langle X \cup\left\{x_{0}, \ldots, x_{n-1}\right\}-\left\{x_{i+1}, x_{j}\right\}\right\rangle\right) \\
&-r\left(\left\langle X \cup\left\{x_{0}, \ldots, x_{n-1}\right\}-\left\{x_{i}, x_{i+1}\right\}\right\rangle \cap\left\langle X \cup\left\{x_{0}, \ldots, x_{n-1}\right\}-\left\{x_{i+1}, x_{j}\right\}\right\rangle\right. \\
&=(r-1)+(r-1)-(r-2) \\
&= r
\end{aligned}
$$

Now suppose $X_{i}, X_{j}$ are inconsecutive. The intersection term will now have rank $r-3$, one less than when they were consecutive, so $r\left(X_{i} \cup X_{j}\right)=r+1$. Note that these two calculations imply the rank of the union of any three $X_{i}$ 's must be $r+1$. We can now show that the inequality above holds:

$$
\sum_{i=3}^{n+1}(r-1)+(r+1)+(r+1)+\sum_{i=4}^{n+1}(r+1)>r+r+\sum_{i=3}^{n+1} r+\sum_{i=4}^{n+1} r
$$

Therefore $X_{1}, \ldots, X_{n+1}$ is a bad family if and only if

$$
\begin{aligned}
& (n+1-2)(r-1)+2(r+1)+(n+1-3)(r+1) \\
& \quad>2 r+(n+1-2) r+(n+1-3) r
\end{aligned}
$$

which is true if and only if

$$
\begin{aligned}
(n-1)(r-1)+n(r+1) & >(2 n-1) r \\
(2 n-1) r+1 & >(2 n-1) r
\end{aligned}
$$


and this completes the proof.

Call this relaxation $N^{\prime}$.

Lemma 7.7. Relaxing $H_{i} \cup\{e, f\}$ in $N^{\prime}$ creates a $\mathbb{K}$-representable matroid for any $i \in\{1, \ldots, n-1\}$.

Proof. Construct $L$ from $M$ in exactly the same way as $N$ was constructed, up until the point where $p$ and $p^{\prime}$ are added. Instead of adding $p$ to $\langle X\rangle$, add it freely to $\left\langle X \cup\left\{x_{1}, \ldots, x_{i}\right\}\right\rangle$. Now add $p^{\prime}$ freely to $\left\langle X \cup\left\{x_{i+1}, \ldots, x_{n-1}, x_{0}\right\}\right\rangle$. Then add $e$ and $f$ freely to the line $\left\langle\left\{p, p^{\prime}\right\}\right\rangle$ as before. This matroid $L$ is $\mathbb{K}$-representable by construction. We will show that it is the same as the matroid obtained from $N^{\prime}$ by relaxing $H_{i} \cup\{e, f\}$, referred to as $N^{\prime \prime}$.

Note that by [7, Proposition 3.3.5], we have that $N \backslash e \backslash f=N^{\prime} \backslash e \backslash f=N^{\prime \prime} \backslash e \backslash f$, and also that $N \backslash e \backslash f=L \backslash e \backslash f$ by construction. If $Z \subseteq E(N \backslash e)$ spans $f$, then, as we chose $f$ to be freely placed on the line spanned by $p$ and $p^{\prime}, Z$ must span $\left\langle\left\{p, p^{\prime}\right\}\right\rangle$. This implies that $p^{\prime} \in\langle Z\rangle$. As $p^{\prime}$ was freely placed in $E(N \backslash e)$, this implies $Z$ is spanning. Thus $N \backslash e$ is a free extension of $N \backslash e \backslash f$ by the element $f$.

Now suppose $Z \subseteq E(L \backslash e)$ spans $f$. Then again we have that $\left\langle\left\{p, p^{\prime}\right\}\right\rangle \subseteq\langle Z\rangle$. As this gives that $p \in\langle Z\rangle$, we have that $X \cup\left\{x_{1}, \ldots, x_{i}\right\} \subseteq\langle Z\rangle$ by the way $p$ was chosen in the construction of $L$. As $p^{\prime} \in\langle Z\rangle$, we have that $X \cup\left\{x_{i+1}, \ldots, x_{n-1}, x_{0}\right\} \subseteq$ $\langle Z\rangle$. Putting these together gives $X \cup\left\{x_{0}, \ldots, x_{n-1}\right\} \subseteq\langle Z\rangle$. As $X \cup\left\{x_{0}, \ldots, x_{n-1}\right\}$ was chosen so as to be a basis of $L, Z$ must be spanning. This tells us that $f$ is freely placed in $L \backslash e$, so $L \backslash e$ is a free extension of $L \backslash e \backslash f$ by the element $f$. As $L \backslash e \backslash f=N \backslash e \backslash f$, we have that $L \backslash e=N \backslash e$. Note also that $N \backslash e=N^{\prime} \backslash e=N^{\prime \prime} \backslash e$, so $L \backslash e=N^{\prime \prime} \backslash e$. The same argument shows that $L \backslash f=N^{\prime \prime} \backslash f$.

Suppose $L \neq N^{\prime \prime}$. There must exist a set $A$ which is a non-spanning circuit in $N^{\prime \prime}$ and independent in $L$ or vice versa. The above two paragraphs imply that $e, f \in A$, as otherwise $A$ would have the same rank in both $L$ and $N^{\prime \prime}$.

Suppose $A$ is a non-spanning circuit in $L$. Say that the points in $E(L)-\{e, f\}$ were added in the order $e_{1}, \ldots, e_{t}$. Let $e_{j}$ be the largest element of $A$ according to this ordering, and let $e_{j} \in H_{k}$. As $e_{j}$ was freely placed, $A$ must span $\langle(X \cup$ $\left.\left.\left\{x_{0}, \ldots, x_{n-1}\right\}\right)-\left\{x_{k}, x_{k+1}\right\}\right\rangle$. This means that

$$
\left(A-H_{k}\right) \cup\left(\left(X \cup\left\{x_{0}, \ldots, x_{n-1}\right\}\right)-\left\{x_{k}, x_{k+1}\right\}\right)
$$


spans the same set as $A$. Suppose the last element added to $A$ before those in $H_{k}$ is $e_{l} \in H_{j}$ where $j<k$. Then $\left(A-H_{k}\right) \cup\left(X \cup\left\{x_{0}, \ldots, x_{n-1}\right\}\right)-\left\{x_{k}, x_{k+1}\right\}$ spans an element from $H_{j}$, and by construction, as every element in the set above was added before $H_{j}$, we see that this set spans $\left(X \cup\left\{x_{0}, \ldots, x_{n-1}\right\}\right)-\left\{x_{j}, x_{j+1}\right\}$. Thus $A$ spans both $\left\langle\left(X \cup\left\{x_{0}, \ldots, x_{n-1}\right\}\right)-\left\{x_{k}, x_{k+1}\right\}\right\rangle$ and $\left\langle\left(X \cup\left\{x_{0}, \ldots, x_{n-1}\right\}\right)-\left\{x_{j}, x_{j+1}\right\}\right\rangle$. As shown in Lemma 7.6, if $H_{j}$ and $H_{k}$ are inconsecutive, $A$ will have rank $r+1$ and be spanning. $H_{j}$ and $H_{k}$ must be consecutive in order for $A$ to be non-spanning. Take a dependent subset of $H_{j} \cup H_{k}$ in $L$. As this subset does not include $e$ nor $f$, it has the same rank in $L \backslash e$. Likewise, the rank of the subset in $N^{\prime \prime}$ has the same rank in $N^{\prime \prime} \backslash e$. As we have already shown $L \backslash e=N^{\prime \prime} \backslash e$, we have that any dependent subset of $H_{j} \cup H_{k}$ in $L$ is also dependent in $N^{\prime \prime}$. This contradicts the assumption that $A$ is independent in $N^{\prime \prime}$. If there is no point contained in a set $H_{j}$ where $j<k$, in order for $A$ to be a circuit, $A$ must be equal to $H_{k} \cup\{e, f\}$, where $k \notin\{0, i\}$, as any subset of this is independent in $L$, as proved in the next lemma.

Lemma 7.8. $H_{k} \cup\{e, f\}$ is a circuit of $L$ for all $k \in\{1, \ldots, i-1, i+1, \ldots, n\}$.

Proof. Consider $H_{k} \cup\{e, f\}$. Recall that $p$ was added freely to $\left\langle X \cup\left\{x_{1}, \ldots, x_{i}\right\}\right\rangle$ while $p^{\prime}$ was added freely to $\left\langle X \cup\left\{x_{i+1}, \ldots, x_{n-1}, x_{0}\right\}\right\rangle . H_{k}$ is contained in $\langle(X \cup$ $\left.\left\{x_{0}, \ldots, x_{n-1}\right\}-\left\{x_{k}, x_{k+1}\right\}\right\rangle$. When $i \leq k$, this subspace spans $\left\langle X \cup\left\{x_{1}, \ldots, x_{i}\right\}\right\rangle$ and so spans $p$. When $i \geq k$, this subspace spans $\left\langle X \cup\left\{x_{i+1}, \ldots, x_{n-1}, x_{0}\right\}\right\rangle$ and so spans $p^{\prime}$. As $e$ and $f$ were freely placed on the line spanned by $p$ and $p^{\prime}$, in either case we have that $H_{k} \cup\{e, f\} \in \operatorname{cl}\left(H_{k} \cup\{e\}\right)$ and so $H_{k} \cup\{e, f\}$ is dependent.

Suppose $H_{k}$ is dependent for some $k$. Then at some point in constructing $L$, we would have added a point $g$ to already chosen elements of $H_{k}$ so that the point was contained in $\operatorname{cl}\left(H_{k}-g\right)$, that is, contained in $\left\langle\left(X \cup\left\{x_{0}, \ldots, x_{n-1}\right\}\right)-\left\{x_{k}, x_{k+1}\right\}\right\rangle$. This contradicts each of the $r-1$ points of $H_{k}$ being freely placed in the rank $r-1$ space $\left\langle\left(X \cup\left\{x_{o}, \ldots, x_{n-1}\right\}\right)-\left\{x_{k}, x_{k+1}\right\}\right\rangle$. Thus $H_{k}$ is independent.

Now suppose $H_{k} \cup\{e\}$ is dependent. Then $e \in \operatorname{cl}\left(H_{k}\right)$ - that is, $e \in\langle(X \cup$ $\left.\left.\left\{x_{0}, \ldots, x_{n-1}\right\}\right)-\left\{x_{k}, x_{k+1}\right\}\right\rangle$. However, $e$ was freely placed on the line spanned by $p$ and $p^{\prime}$. Thus if $H_{k}$ spans $e$, it must span this line. As the line itself is free in $L$, for this to happen, $H_{k}$ must be spanning, which is a contradiction. Likewise, $H_{k} \cup\{f\}$ is also independent. We have shown that every subset of $H_{k} \cup\{e, f\}$ is independent, meaning that $H_{k} \cup\{e, f\}$ must be a circuit.

Thus $H_{k} \cup\{e, f\}$ is dependent in $L$. We have shown that it is also dependent in $N^{\prime \prime}$, 
so again have a contradiction to $A$ being independent in $L$. The same argument shows that if $A$ is dependent in $N^{\prime \prime}, A$ is also dependent in $L$. Thus $L=N^{\prime \prime}$.

We constructed $N$ to be representable, so $N$ must satisfy every Kinser inequality. In particular, it must be contained inside $\mathcal{K}_{n+1}$. Next we have shown in Lemma 7.7 that if we relax a single circuit-hyperplane of $N$, the resulting matroid $N^{\prime}$ has a bad family for $\mathcal{K}_{n+1}$. We will now show that $N^{\prime}$ is in fact an excluded minor for $\mathcal{K}_{n+1}$ - that is, we will show that each proper minor of $N^{\prime}$ is representable and thus is contained in $\mathcal{K}_{n+1}$.

First suppose that $x \in H_{j}$ where $j \neq 0$. Let $N^{\prime \prime}=N^{\prime}$ with the circuit-hyperplane $H_{j} \cup\{e, f\}$ relaxed. By [7, Proposition 3.3.5], $N^{\prime \prime} \backslash x=N^{\prime} \backslash x$. As $N^{\prime \prime}$ is $\mathbb{K}$ representable by Theorem 7.7, and representability is preserved under minors, $N^{\prime} \backslash x$ is $\mathbb{K}$-representable. Say $l \in\{0, \ldots, n-1\}-\{0, j\}$. Now let $N^{\prime \prime}=N^{\prime}$ with $H_{l} \cup\{e, f\}$ relaxed. Also by [7, Proposition 3.3.5], we have that $N^{\prime \prime} / x=N^{\prime} / x$, and so $N^{\prime} / x$ is $\mathbb{K}$-representable.

Next, suppose $x \in H_{0}$. As $N^{\prime}=N$ with the circuit-hyperplane $H_{0} \cup\{e, f\}$ relaxed, we have that $N^{\prime} \backslash x=N \backslash x$, so $N^{\prime} \backslash x$ is $\mathbb{K}$-representable. Let $N^{\prime \prime}=N^{\prime}$ with $H_{i} \cup\{e, f\}$ relaxed. We have that $N^{\prime \prime} / x=N^{\prime} / x$, so $N^{\prime} / x$ is $\mathbb{K}$-representable.

Now suppose $x$ is equal to $e$. As $e$ and $f$ were freely placed on the line spanned by $p$ and $p^{\prime}$, the same argument as follows works for $x=f$. We have that $N^{\prime} \backslash e=N \backslash e$, so $N^{\prime} \backslash e$ is $\mathbb{K}$-representable.

Finally, consider $N^{\prime} / e$. Take some $z \in H_{0}$. Recall that $N^{\prime}=N$ with the circuithyperplane $H_{0} \cup\{e, f\}$ relaxed. Note that $N^{\prime} / e$ is obtained from $N / e$ by relaxing $H_{0} \cup\{f\}$. This gives us that $N^{\prime} / e \backslash z=N / e \backslash z$, as deleting $z$ effectively undoes the relaxation. As $N$ is $\mathbb{K}$-representable, and thus $N / e \backslash z$ is $\mathbb{K}$-representable, $N^{\prime} / e \backslash z$ is also $\mathbb{K}$-representable. Let $Z \subseteq E\left(N^{\prime} / e\right)$ be such that $z \notin Z$ and $z \in \mathrm{cl}_{N^{\prime} / e}(Z)$. Recall $N^{\prime} / e$ is a relaxation of $N / e$ which can only affect closures in so far as that some may contain additional elements in $N / e$, so $z \in \mathrm{cl}_{N / e}(Z)$. This implies that $z \in \mathrm{cl}_{N}(Z \cup\{e\})$ by [7, Proposition 3.1.11]. Due to the way $H_{0}$ was constructed, we thus have that $\langle Z \cup\{e\}\rangle \supseteq\left(X \cup\left\{x_{0}, \ldots, x_{n-1}\right\}\right)-\left\{x_{0}, x_{1}\right\}$. As we have that $z \in \operatorname{cl}_{N}(Z \cup\{e\})$ and all elements of $H_{0}$ are freely placed in the relevant subspace, $Z \cup\{e, f\}$ must thus also span every other element of $H_{0}$. As $e$ and $f$ were freely placed on the line spanned by $p$ and $p^{\prime}$, we also have that $f \in \operatorname{cl}_{N}(Z \cup\{e\})$. Thus in $N, H_{0} \cup\{e, f\}$ is contained in $\langle Z \cup\{e\}\rangle$. As $H_{0} \cup\{e, f\}$ is a circuit-hyperplane, this implies that either $Z \cup\{e\}$ is spanning in $N$ or that $Z \cup\{e\}=H_{0} \cup\{e, f\}$. If 
$Z \cup\{e\}=H_{0} \cup\{e, f\}$, we have a contradiction to the assumption that $z \notin Z$. We thus have that $Z \cup\{e\}$ is spanning in $N$. This means that $Z \cup\{e\}$ is also spanning in $N^{\prime}$, and, as $r\left(N^{\prime} / e\right)=r\left(N^{\prime}\right)-1$, that $Z$ is spanning in $N^{\prime} / e$. We have that $z$ is only in the closure of a subset of $N^{\prime} / e$ when that subset spans $N^{\prime} / e$ - that is, we have shown that $z$ is freely placed in $N^{\prime} / e$. Thus $N^{\prime} / e$ is a free extension of $N^{\prime} / e \backslash z$ by $z$. As $N^{\prime} / e \backslash z$ is $\mathbb{K}$-representable and this fact is preserved under free extensions, we have that $N^{\prime} / e$ is $\mathbb{K}$-representable.

We have now shown that every minor of $N^{\prime}$ is $\mathbb{K}$-representable and so contained in $\mathcal{K}_{n+1}$, making $N^{\prime}$ an excluded minor for $\mathcal{K}_{n+1}$. This completes the proof of Theorem 7.1 


\section{Chapter 8}

\section{Conjectures}

Finally, we give some conjectures on the hierarchy of the Kinser classes.

Conjecture 8.1. Let $n>5$. $\mathcal{K}_{n} \neq \mathcal{K}_{n}^{*}$.

Recall that we have proved this holds when $n=5$ in Theorem 5.1, and have proved this does not hold when $n=4$ in Theorem 4.5 ,

As shown in Theorem 6.3, verifying that a matroid satisfies a Kinser inequality is very difficult. Given the amount of difficulty involved in proving that the fifth Kinser class is not dual closed, proving this result in general would involve an even greater amount of work. Based on that case, however, we give a strengthening of the above conjecture.

Conjecture 8.2. Let $n \geq 5$. $\operatorname{Kin}(n)^{-} \in \mathcal{K}_{n}^{*}-\mathcal{K}_{n}$

One further question about the structure of the hierarchy is how each dual class interacts with the previous Kinser class. There are two possibilities here, and we conjecture that the following is true.

Conjecture 8.3. Let $n>4$. $\mathcal{K}_{n+1}^{*} \subseteq \mathcal{K}_{n}$.

If Conjecture 8.3 is true, the following conjecture is also true.

Conjecture 8.4. $\mathcal{K}_{\infty}^{*}=\mathcal{K}_{\infty}$

To see that this follows from Conjecture 8.3 , assume $M \in \mathcal{K}_{\infty}$, but $M \notin \mathcal{K}_{\infty}^{*}$ and assume that Conjecture 8.3 holds. Then there exists an integer $n$ such that $M \notin \mathcal{K}_{n}^{*}$. However, this contradicts Conjecture 8.3 , which gives that $M \in \mathcal{K}_{n+1} \subseteq \mathcal{K}_{n}^{*}$. 


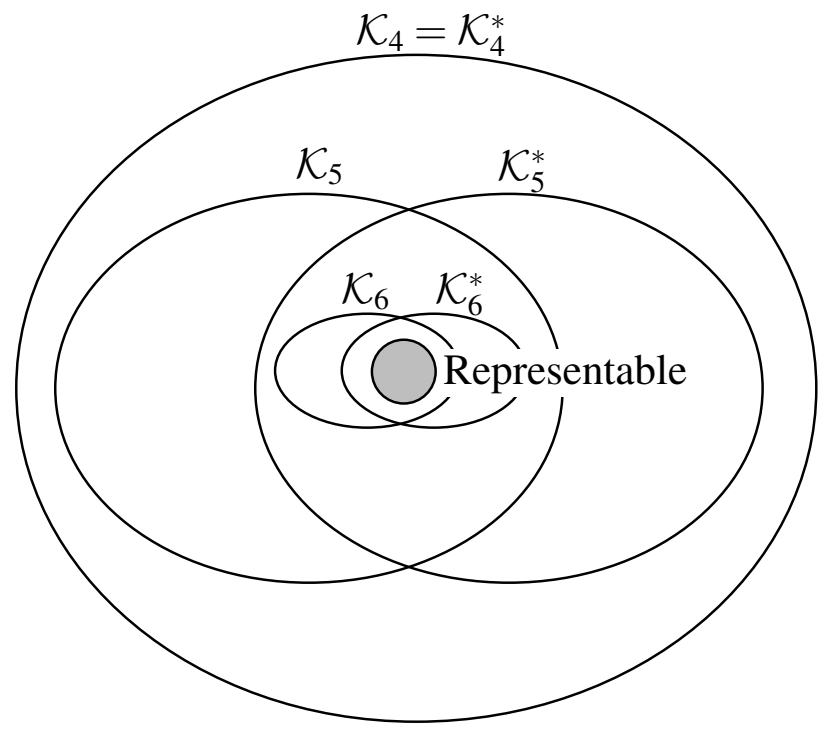

Figure 8.1: Kinser classes (4)

Assuming these conjectures to hold true, we have a final diagram of the hierarchy.

Now we will consider two classes of matroids which we conjecture satisfy every Kinser inequality.

Definition 8.5. Let $G$ be an abelian group. Take a complete graph and add a loop to very vertex, replace every edge with a parallel class of $|G|$ edges. Call this graph $H$. Orient every edge which is not a loop so that parallel edges have the same direction. Bijectively label each parallel class with the elements of $G$, and label loops with non-identities. Let $C$ be a cycle of $H$. Consider the product of group labels in $C$, taken in cyclic order, where if an edge is oriented against the cyclic order we take the inverse of its label instead. If the result is the identity, call $C$ positive. Otherwise, call $C$ negative. Take such a graph $H$. There exists a matroid which has $E(H)$ as its ground set, and set of circuits equal to the positive cycles of $H$ and minimal connected subgraphs that contain two negative cycles. Call this matroid a Dowling geometry.

Take a field $\mathbb{F}$. Recall that $\mathbb{F}^{\times}$is the multiplicative group consisting of the nonzero elements of $\mathbb{F}$.

Lemma 8.6 ([7, Theorem 6.10.10]). Take a Dowling geometry of rank $r$ over a finite group $G$. This matroid is representable over a field $\mathbb{F}$ if and only if $G$ is isomorphic to a subgroup of $\mathbb{F}^{\times}$. 
In this case, the Dowling matroid satisfies every Kinser inequality. We also have that if $G$ is a finite subgroup of the multiplicative group of a field, then $G$ is cyclic by [1, Theorem 33.4]. The following conjecture is thus open when $G$ is both finite and non-cyclic.

Conjecture 8.7. A Dowling geometry satisfies every Kinser inequality.

Now we will consider matroids which are representable over skew partial fields. All of the following definitions and results can be found in [8].

Definition 8.8. A skew partial field is a pair $(R, G)$ where $R$ is a ring, and $G$ is a subgroup of the group of units of $R$, such that $-1 \in G$.

Definition 8.9. Let $R$ be a ring, and let $E$ be a finite set. An $R$-chain group on $E$ is a subset $C \subseteq R^{E}$ such that, for all $f, g \in C$ and $r \in R$,
i. $0 \in C$,
ii. $f+g \in C$,
iii. $r f \in C$

The elements of $C$ are called chains, and the support of a chain $c=\left\{c_{1}, \ldots, c_{e}\right\} \in$ $C$ is

$$
\|c\|=\left\{i \in E \mid c_{i} \neq 0\right\}
$$

Definition 8.10. A chain $c \in C$ is elementary if $c \neq 0$ and there is no $c^{\prime} \in C-\{0\}$ with $\left\|c^{\prime}\right\| \subset\|c\|$.

Definition 8.11. Let $G$ be a subgroup of the group of units of $R$. A chain $c \in C$ is $G$-primitive if $c \in(G \cup\{0\})^{E}$.

Definition 8.12. Let $\mathbb{P}=(R, G)$ be a skew partial field, and $E$ a finite set. A $\mathbb{P}$ chain group on $E$ is an $R$-chain group $C$ on $E$ such that every elementary chain $c \in C$ can be written as $c=r c^{\prime}$ for some $G$-primitive chain $c^{\prime} \in C$ and some $r \in R$.

Lemma 8.13. Let $\mathbb{P}=(R, G)$ be a skew partial field, and let $C$ be a $\mathbb{P}$-chain group on $E$. Then $\mathcal{C}^{*}=\{\|c\| \mid c \in C, c$ is elementary $\}$ is the set of cocircuits of a matroid on $E$. 
A matroid $M$ is said to be $\mathbb{P}$-representable if there exists a $\mathbb{P}$-chain group $C$ such that $M=M(C)$. If a matroid is representable and thus satisfies every Kinser inequality, it is representable over a skew partial field.

Conjecture 8.14. Take a matroid $M$ which is $\mathbb{P}$-representable. M satisfies every Kinser inequality. 


\section{Bibliography}

[1] Fraleigh, J. B. A First Course in Abstract Algebra, seventh ed. AddisonWesley Publishing Co., Reading, Mass. -London-Don Mills, O., 2002.

[2] GeElen, J. Some open problems on excluding a uniform matroid. Adv. in Appl. Math. 41, 4 (2008), 628-637.

[3] Ingleton, A. Conditions for representability and transversality of matroids. Théorie des Matrö̈des (1971), 62-66.

[4] Kinser, R. New inequalities for subspace arrangements. J. Combin. Theory Ser. A 118, 1 (2011), 152-161.

[5] Mayhew, D., Newman, M., And Whittle, G. Is the missing axiom of matroid theory lost forever? Submitted.

[6] Mayhew, D., Newman, M., And Whittle, G. On excluded minors for real-representability. J. Combin. Theory Ser. B 99, 4 (2009), 685-689.

[7] OXLEy, J. Matroid theory, second ed. Oxford Graduate Texts in Mathematics. Oxford University Press, Oxford, 2011.

[8] Pendavingh, R., And Van Zwam, S. Skew partial fields, multilinear representations of matroids, and a matrix tree theorem. Adv. in Appl. Math. 50, 1 (2013), 201-227.

[9] VÁmos, P. The missing axiom of matroid theory is lost forever. J. London Math. Soc. (2) 18, 3 (1978), 403-408.

[10] Whitney, H. On the Abstract Properties of Linear Dependence. Amer. J. Math. 57, 3 (1935), 509-533. 